\title{
Tissue-specific H2Bub1 is required for cardiomyocyte development
}

\section{Syndi Barish}

Jeffrey Drozd

Yale University School of Medicine

Kathryn Berg

Yale University School of Medicine

Isabella Berglund-Brown

Yale University School of Medicine

\section{Labeeqa Khizir}

Yale University School of Medicine

\section{Lauren Wasson}

Harvard Medical School https://orcid.org/0000-0002-5193-5215

\section{Sidi Chen}

Yale University https://orcid.org/0000-0002-3819-5005

Martina Brueckner ( $\square$ martina.brueckner@yale.edu )

Yale University School of Medicine https://orcid.org/0000-0003-0347-5389

\section{Article}

Keywords: H2Bub1, congenital heart disease, tissue-specific regulation, RNF20-core complex

Posted Date: August 24th, 2021

DOI: https://doi.org/10.21203/rs.3.rs-788134/v1

License: (c) (1) This work is licensed under a Creative Commons Attribution 4.0 International License.

Read Full License 
3 Syndi Barish $^{1}$, Jeffrey Drozd ${ }^{2}$, Kathryn Berg ${ }^{1}$, Isabella Berglund-Brown ${ }^{2}$, Labeeqa Khizir ${ }^{2}$,

4 Lauren K Wasson ${ }^{3,4}$, Sidi Chen ${ }^{1}$, Martina Brueckner ${ }^{1,2, *}$

6 Departments of Genetics ${ }^{1}$ and Pediatrics ${ }^{2}$

7 Yale University School of Medicine,

8333 Cedar Street,

9 New Haven, Connecticut 06510, USA

Department of Genetics ${ }^{3}$,

12 Harvard Medical School

13 Boston, MA 02115, USA

14

Howard Hughes Medical Institute, Division of Cardiovascular Medicine ${ }^{4}$

16 righam and omen s Hospital

17 Boston, MA 02115, USA

18

19

*Correspondence to:

20 Martina.brueckner@yale.edu 


\section{$21 \underline{\text { Abstract }}$}

22 De-novo mutations affecting Histone2B-K120 monoubiquitination (H2Bub1) are enriched in

23 congenital heart disease; however, how H2Bub1 affects heart morphogenesis beyond its function

24 in establishing left-right asymmetry remains unknown. Here we show that the RNF20-core

25 complex (RNF20-RNF40-UBE2B), which catalyzes H2Bub1, is required for cardiac

26 development in-vivo in mice and in-vitro in iPSC-derived cardiomyocytes. Mice with cardiac-

27 specific deletion of Rnf20 have abnormal myocardium and ventricular septal defects; iPSCs with

28 mutations affecting H2Bub1 cannot efficiently differentiate into cardiomyocytes. Sarcomeric

29 gene expression is dependent on normal H2Bub1 both in mice and in iPSC-derived

30 cardiomyocytes. Finally, we identify an accumulation of H2Bub1 near the center of tissue-

31 specific genes in cardiomyocytes and MEFs associated with transcriptional efficiency that is

32 reduced in $U B E 2 B^{-/}$cardiomyocytes. In summary, normal $\mathrm{H} 2 \mathrm{Bub} 1$ distribution is required in

33 cardiac development, and H2Bub1 accumulation acts as a general mechanism for tissue-specific

34 regulation of transcriptional elongation efficiency. 


\section{$\underline{\text { Introduction }}$}

Congenital heart disease (CHD), a structural abnormality of the heart and/or great

37 vessels, is the most common cause of mortality from congenital malformations. Although $90 \%$

38 of patients survive to adulthood, many suffer from co-morbidities including myocardial

39 dysfunction and neurodevelopmental abnormalities ${ }^{1}$. Whole-exome sequencing of CHD patients

40 identified variants in a broad spectrum of chromatin modifier genes in $2.3 \%$ of cases (including

$41 \mathrm{H} 3 \mathrm{~K} 4$ and H3K37 methylation and H2BK120 monoubiquitination) $)^{2-4}$. Chromatin modifiers have

42 previously been linked to heart development through animal models. Knock-out models of

43 chromatin modifiers, such as $\mathrm{Kmt2d}$ (H3K4 methyltransferase), Dot1l (H3K79

44 methyltransferase), and Hdac1/Hdac2 (histone deacetylases), result in a range of congenital heart

45 abnormalities in vertebrates ${ }^{5-8}$. Further, many chromatin marks, including H3K4me3 and

$46 \mathrm{H} 3 \mathrm{~K} 27 \mathrm{me}$, are dynamic throughout cardiac lineage commitment. The correlation of chromatin

47 patterns with stage-specific gene expression suggests that normal regulation of chromatin

48 modifications is important for cardiomyocyte development ${ }^{9}$.

Although several chromatin modifiers have been directly linked to heart development,

50 how monoubiquitination of histone H2B on K120 (H2Bub1) affects cardiogenesis is largely

51 unknown. The H2Bub1 machinery was first discovered in yeast; $\mathrm{H} 2 \mathrm{~B}$ is monoubiquitinated by

52 Bre1 (ubiquitin ligase), Rad6 (ubiquitin-conjugating enzyme), and Lge1 (scaffolding protein) ${ }^{10-}$

$53{ }^{12}$. In mammals, the E3 ubiquitin ligases RNF20 and RNF40 correspond to Bre1, the Ubiquitin

54 Conjugating enzyme E2 B (UBE2B) corresponds to Rad6, and the WW Domain-containing

55 adaptor with coiled-coil (WAC) corresponds to Lge ${ }^{13-16}$. Unlike most histone marks, H2Bub1 is

56 located on gene bodies, where it is enriched near the promoter and gradually decreases towards

57 the 3 end $^{17}$. In order for the complex to add H2Bub1 in-vitro, Bre1 and Rad6 form a catalytic 
58 shell around Lge1 enabling a liquid-liquid phase separation, which then recruits the nucleosome.

59 If the ratios of the complex components change, so does its efficiency ${ }^{16}$. Thus, it is hypothesized

60 that in-vivo in mammals, the ratios of the components affect the efficiency of the complex, and

61 thus differential component expression can dictate where H2Bub1 is added.

62 CHD patients show an enrichment in de novo mutations affecting the RNF20

63 interactome, compared to controls, demonstrating a clinical relevance of H2Bub1 in CHD.

64 Presently, the only known cardiac roles of H2Bub1 are in regulating cardiac situs by controlling

65 cilia gene expression at the left-right organizer and in post-natal cardiomyocyte maturation ${ }^{18,19}$.

66 However, H2Bub1 has broad biological functions including differentiation, tumor suppression,

67 and inflammation ${ }^{20-26}$. H2Bub1 is likely an activating mark, since it interacts with the

68 Set1/COMPASS methyltransferase complex, which in most chromatin contexts creates

69 activating marks ${ }^{27}$. However, in Rnf40 knockouts, there are genes near regions that lost H2Bub1

70 and also have increased expression, suggesting H2Bub1 can also be repressive ${ }^{28}$. Further studies

71 suggest that gene activation requires both the addition and removal of $\mathrm{H} 2 \mathrm{Bub} 1^{29}$. These

72 conflicting results indicate that the effect of $\mathrm{H} 2 \mathrm{Bub} 1$ on transcriptional regulation is still not well

73 understood.

74 H2Bub1 is deposited co-transcriptionally and co-localizes with RNA Pol II. As a result,

75 H2Bub1 is highly correlated with transcriptional elongation, hinting at a positive regulatory

76 mechanism $^{30}$. In yeast, the Paf1 complex (positive regulator of transcriptional elongation) has a

77 direct and specific interaction with Bre ${ }^{31}$. H2Bub1 also positively regulates the histone

78 chaperone FACT, which promotes transcriptional elongation ${ }^{32}$. Conversely, RNF20 inhibits

79 recruitment of TFIIS (which prevents RNA Pol II from stalling), effectively blocking

80 transcriptional elongation ${ }^{23}$. This suggests that H2Bub1 may also negatively affect 
81 transcriptional elongation. Interestingly, increased H2Bub1 levels, due to mutations affecting

82 deubiquitination, result in embryonic lethality in mice, but do not affect global Pol II

83 transcription elongation in mouse embryonic stem cells (mESCs) or mouse embryonic

84 fibroblasts (MEFs) ${ }^{33}$. Thus, as with transcriptional regulation, the role of $\mathrm{H} 2 \mathrm{Bub} 1 \mathrm{on}$

85 transcriptional elongation may be context-dependent.

86 Here we present analysis on the role of H2Bub1 in cardiac morphogenesis beyond its

87 known role in establishing left-right asymmetry, both in-vivo in mouse and in-vitro in human

88 iPSC-derived cardiomyocytes. Deletion of Rnf20 in mouse hearts leads to embryonic lethality,

89 abnormal compact myocardium, disorganized cardiac sarcomeres lacking an $\mathrm{H}$ zone, and

90 ventricular septal defects. We further show that the stoichiometric ratios of the RNF20-complex

91 components affect the deposition of H2Bub1, which limits cardiomyocyte differentiation in-

92 vitro. $\mathrm{H} 2 \mathrm{Bub1}$ is abundant in iPSCs before becoming selectively maintained on a subset of genes

93 significantly enriched in sarcomeric calcium genes during the transition from cardiac mesoderm

94 to cardiac progenitor; this same set of genes is misregulated when $\mathrm{H} 2 \mathrm{Bub} 1$ is decreased in-vitro

95 and in-vivo. Finally, we establish the importance of H2Bub1 localization within a subset of genes

96 notable for tissue-specific expression. We show that the location of H2Bub1 on these long genes

97 is correlated with their transcriptional efficiency. Together, our data indicate that H2Bub1 is

98 essential for cardiac development through regulation of cardiac sarcomeric genes during

99 cardiomyocyte differentiation and development. 


\section{Rnf20 is required for mouse development}

Since RNF20 variants have been linked to human CHD both with and without abnormal

103 laterality, we asked how loss of RNF20 affects cardiogenesis in mice. Analysis of RNF20 protein

104 in mouse embryonic hearts demonstrated ubiquitous cardiac expression at e9.5 (Fig. S1a) that

105 becomes gradually restricted to only the epicardium at birth (Fig. S1b). Myocardial RNF20

106 forms an expression gradient with higher RNF20 at the myocardial surface compared to the

107 lumen, and disappears by e16.5 (Fig. S1c). Endocardial RNF20 also disappears by e16.5 (Fig.

108 S1d). The human CHD-associated mutation in RNF20 is an early stop codon, suggesting

109 haploinsufficiency for $R N F 20$, so we analyzed constitutively heterozygous $R n f 20$ mice

110 containing a targeted deletion of Rnf20 by replacing all coding exons with a LacZ reporter

111 (obtained from KOMP2) (Fig. S2a) ${ }^{18,34}$. The $R n f 20^{+/-}$mice are phenotypically normal and

112 survive to adulthood with no discernible cardiac abnormalities (Fig. S2b). However, no Rnf $20^{-/-}$

113 embryos are recovered post-blastocyst stage, consistent with previous data showing RNF20 is

114 required for preimplantation development $(\text { Fig. 1a, S2b })^{25}$.

115 To test whether RNF20 is required in cardiac development, we generated mice with

116 cardiac-specific deletions of Rnf20 using a conditional $R n f 20^{\mathrm{fl}}$ allele containing loxP sites

117 flanking exons 2-4 of the Rnf20 gene ${ }^{35}$. Since the heart is formed by two waves of cell migration

118 (first-heart field (FHF) predominantly contributing to the atria and left ventricle, and second-

119 heart field (SHF) predominantly contributing to the outflow tract), we made FHF and SHF

120 specific Rnf20 deletion mouse lines using $N k x 2.5 \mathrm{Cre}$ (FHF) and IsllCre (SHF) drivers (Fig.

121 S2c) ${ }^{36,37}$. We evaluated timing and specificity of Cre expression by mating with ROSA ${ }^{\mathrm{mt} / \mathrm{mg}}$ and

122 evaluating Cre expression (Fig. 1a). Rnf $20^{\mathrm{fl} /}:$ : $I$ sll $1 \mathrm{Cre}^{+}$mice survive to at least 7 months at 
123 Mendelian ratios (Fig.1a, S2d). They also have a normal heart/body weight ratio and hearts

124 appear morphologically normal by H\&E staining (Fig. S2e, S2f).

125 NKX2.5 is a transcription factor initially expressed in the entire heart, and then becomes

126 restricted to the $\mathrm{FHF}^{36} . \operatorname{Rnf} 20^{\mathrm{fl} /-}:: N k x 2.5 \mathrm{Cre}^{+}$embryos are found at Mendelian ratios until e12.25,

127 but none were recovered after e12.5 (Fig. 1a, S2h). At e11.5, the embryos and heart appear

128 normal by H\&E, including 100\% normal heart looping direction (Fig. S2g). By e12.25, the

129 compact myocardium is significantly thinned in $\operatorname{Rnf} 20^{\mathrm{fl} /-}:: N k x 2.5 \mathrm{Cre}^{+}$embryos irrespective of

130 comparable diameters to wildtype, and they exhibit large ventricular septal defects (left ventricle

131 compact myocardium thickness $\mathrm{p}$-value $=0.009$, right ventricle compact myocardium thickness

132 p-value $=0.004$, septum length p-value $=0.0007)($ Fig. 1c $)$. We verified the $N k x 2.5$-specific

133 deletion of $R n f 20$ at e11.5; the RNF20 protein is in both myocardial and epicardial cells in the

$134 N k x 2.5 \mathrm{Cre}^{-}$embryos, but only in the NKX2.5- epicardial cells in $N k x 2.5 \mathrm{Cre}^{+}$embryos (Fig. 1b).

135 While the majority of NKX2.5-expressing cells become cardiomyocytes, it remains possible that

136 the structural defect phenotype results from a role for RNF20 in NKX2.5 expressing cardiac

137 tissue including a subset of endocardial cells. Together, these data indicate that RNF20 is

138 required in heart development in the FHF (NKX2.5-expressing cells), but is dispensable in the

139 SHF (ISL1-expressing cells).

141 H2Bub1 is dynamically distributed during cardiomyocyte differentiation

142 Based on our data showing abnormal development of compact myocardium in response

143 to tissue-specific Rnf20 deletion, we investigated H2Bub1 during cardiomyocyte development

144 using in-vitro differentiation of human induced pluripotent stem cells (iPSCs) into

145 cardiomyocytes (CMs) (online methods) ${ }^{38}$. To evaluate the genome-wide H2Bub1 profile and 
146 subsequent transcriptional changes during CM development, ChIP-seq of H2Bub1 and bulk

147 RNA-seq were performed simultaneously at five stages of CM differentiation: iPSCs, mesoderm

$148(\mathrm{M})$, cardiac mesoderm (CMes), cardiac progenitor (CP) and CM. The stages of differentiation

149 were verified through RNA-seq (Fig. S3a, Supplemental Data 2). H2Bub1 ChIP-seq peaks

150 from iPSCs were grouped into four clusters based on H2Bub1 occupancy (high, moderate, low,

151 and none) (Fig. 2b, Supplemental Data 1). ChIP-seq peaks from the other stages are displayed

152 based on these clusters. The general profile of H2Bub1 is as previously reported, with very low-

153 occupancy at the transcription start site (TSS) and coverage over the entire gene-body with

154 gradual diminution rom 5 to $3^{17}$. The moderate-occupancy cluster has a distinct profile

155 compared to the high and low-occupancy clusters. While the high and low-occupancy clusters

156 have constant $\mathrm{H} 2 \mathrm{Bub} 1$ throughout the 5 region o the gene-body, the moderate-occupancy

157 cluster has a drop-off in H2Bub1 occupancy (Fig. 2b). Further, H2Bub1 occupancy increases

158 between iPSC and M near the TSS, but remains constant between M and CMes. It then decreases

159 between CMes and CP, and again remains constant between CP and CM (Fig. 2a).

160 We next performed DAVID gene ontology (GO) enrichment analysis on the genes in

161 each cluster for each cell type to obtain their biological context. The high and moderate-

162 occupancy clusters (Clusters 1 and 3) are enriched for general cell maintenance terms, showing a

163 general need for H2Bub1 in maintaining homeostasis. The no-occupancy cluster (Cluster 4) is

164 enriched for both general cell maintenance and chromatin assembly terms. Most interesting to us

165 is the low-occupancy cluster (Cluster 2), which is enriched in development GO terms. Cluster 2

166 in iPSCs contains multiple types of developmental genes (muscle, renal, eye, heart, etc.),

167 consistent with previous studies demonstrating RNF20 is required to exit pluripotency ${ }^{21,22}$.

168 Strikingly, as cells commit to a cardiac fate (in CP and CM), H2Bub1 is retained only on 
developmental genes related to heart, nervous system, or appendage development (Fig. S3b, S3c,

\section{Supplemental Data 1).}

171 Total H2Bub1 increases from CMes to CP, in contrast to the ChIP-seq data, which shows

172 a decrease in $\mathrm{H} 2 \mathrm{Bub} 1$ around gene bodies during the equivalent stages of CM differentiation.

173 Total H2Bub1 in the other stages agree with the ChIP-seq data (Fig. 2a, 2d, S3d). One

174 explanation is that $\mathrm{H} 2 \mathrm{Bub} 1$ is decreasing in occupancy around the gene-body, but increasing in

175 occupancy in heterochromatic regions. To test this, we compared the amount of H2Bub1 in the B

176 compartment (heterochromatic compartment) to expected values calculated by generating

177 bootstrap replicates from previously published data at each cell stage (online methods) $)^{39}$. We

178 found $\mathrm{H} 2 \mathrm{Bub} 1$ first significantly decreases in the B compartment between iPSC and M, before

179 significantly increasing as the cells progress through all the stages from M to CM (p-values

$\left.180<1 \mathrm{X} 10^{-5}\right)$. By the CM stage, there is significantly more H2Bub1 in the B compartment than

181 expected by chance (p-values $<1 \times 10^{-5}$ ) (Fig. S3e). The gradual transition of H2Bub1 from gene

182 bodies to heterochromatin is further supported by a significantly higher overlap of H2Bub1 and

183 previously published $\mathrm{H} 3 \mathrm{~K} 27 \mathrm{me} 3$ peaks (heterochromatic mark) on the same gene in CMs

184 compared to iPSCs ( $\mathrm{p}$-values $<1 \times 10^{-5}$ ), versus no change in overlap of H2Bub1 and previously

185 published H3K4me3 peaks (active genes) ${ }^{40,41}$. Further, in CMs, there is a significantly higher

186 overlap of $\mathrm{H} 2 \mathrm{Bub} 1$ and $\mathrm{H} 3 \mathrm{~K} 27 \mathrm{me} 3$ peaks on the same gene than of H2Bub1 and H3K4me3

187 peaks (p-value $=0.04)$. In iPSCs, both overlaps are equivalent $($ Fig. S3f). We conclude that the

188 percent of $\mathrm{H} 2 \mathrm{Bub} 1$ marks in active regions remains constant between iPSCs and CMs, but the

189 percent of heterochromatic regions marked by H2Bub1 increases between iPSCs and CMs so

190 that there are more heterochromatic regions marked by H2Bub1 than active regions at the CM

191 stage. Together these data indicate that H2Bub1 increases in heterochromatic regions, which 
192 accounts for the discrepancy between the observed increase in total H2Bub1 from CMes to CP,

193 while gene-specific H2Bub1 decreases at the same stages.

195 H2Bub1 is selectively maintained on sarcomeric calcium genes

The distribution of many epigenetic marks is dynamic during development ${ }^{9,42}$. To

197 determine the temporal dynamics of H2Bub1 occupancy during cardiomyocyte differentiation,

198 differential binding analysis on the H2Bub1 ChIP-seq data was performed during the progression

199 from iPSCs to CMs. We found 316 regions that have increased H2Bub1 between iPSC and M,

200 compared to 18 regions with decreased H2Bub1. Between M and CMes, there are 2 regions with

201 decreased H2Bub1. The largest change in H2Bub1 happens upon transition from CMes to CP,

202 with a decrease in 25,748 regions, while no gene-specific changes in H2Bub1 between CP and

203 CM were observed (Fig. 2f, Supplemental Data 1). This drop off in H2Bub1 occupancy

204 between CMes and CP is further shown in a Venn diagram comparing the unique CMes Ensembl

205 genes near H2Bub1 regions to the unique CP genes (Fig. 2e). This is consistent with the

206 overview data (Fig. 2a). DAVID GO enrichment analyses on regions that change in H2Bub1

207 occupancy, based on the average of all three replicates, indicate that they are mostly cell

208 maintenance genes (Fig. 2g). Since this mark is thought to be activating, we verified expression

209 of the genes near H2Bub1 for all cell stages. We evaluated the overlap of ChIP-seq and RNA-seq

210 results in the terminal $\mathrm{CM}$ stage in more detail and identified 432 genes that are predicted to be

211 direct targets of H2Bub1 (Fig. 2c). Consistent with a role for H2Bub1 in CMs, DAVID GO

212 analysis of these 432 genes identified a significant enrichment ( $p$-value $=0.014$ ) in cardiac

213 conduction genes, including the calcium channel CACNAIC. 
215 the genes that maintain their mark during the large drop off in H2Bub1 occupancy between

216 CMes and CP are also cardiac genes. Genes that maintain H2Bub1 in CP are enriched for

217 calcium signaling genes (Fig. 2h). Interestingly, calcium signaling genes remain constant in

218 H2Bub1 occupancy over time, unlike housekeeping genes which follow the general trend

219 outlined in Fig. 2a (Fig. 2i). Further, a significantly higher proportion of calcium signaling genes

220 is identified within the genes that have maintained H2Bub1 compared to genes with

221 downregulated H2Bub1 $\left(\mathrm{Z}\right.$ score $=4.3996$, p-value $=1.1$ X 10 $\left.0^{-5}\right)$. Importantly, ten of the eleven

222 calcium signaling genes that maintain $\mathrm{H} 2 \mathrm{Bub} 1$ are associated with cardiomyopathy either

223 through patient mutations or mouse models (Fig. 2h) ${ }^{43-52}$. These include CACNA1C and RYR2

224 which have been linked to left ventricular non-compaction (LVNC) ${ }^{51,52}$. Together, these data

225 indicate that $\mathrm{H} 2 \mathrm{Bub} 1$ is selectively maintained on tissue-specific genes in CMs to promote their

226 expression.

227

228 Patterns in H2Bub1 occupancy predict gene expression

229 The distribution of chromatin marks, such as H3K4me3, is known to affect gene

230 expression, so we wanted to determine if the same is true for $\mathrm{H} 2 \mathrm{Bub} 1^{28}$. We next asked if we

231 could identify patterns of H2Bub1 distribution and amount on CMes marked genes that we could

232 use to predict the subsequent loss of $\mathrm{H} 2 \mathrm{Bub} 1$ and gene expression in CP. By using a previously

233 established method, we identified multiple groups of genes (patterns) that have similar

234 distribution or amount of H2Bub1 across the gene body (Fig. S4a, Supplemental Data 1$)^{53}$.

235 Interestingly, the calcium signaling genes were among the many genes that did not get placed

236 into any pattern, hereafter referred to as unpatterned genes . 
We next calculated the ratio of H2Bub1 peaks that decrease in occupancy between CMes 238 and $\mathrm{CP}$ to total $\mathrm{H} 2 \mathrm{Bub} 1$ peaks for each of these patterns. Patterned genes have a higher ratio of

239 peaks decreasing in occupancy than average (Fig. S4b). On the contrary, some unpatterned 240 genes, such as the calcium signaling genes, maintain their H2Bub1 occupancy. The decreased

241 occupancy in patterned genes corresponds to their significantly decreased expression in CP

242 compared to CMes (all p-values < 0.05). Thus, if H2Bub1 only occupies a particular region of a

243 gene body (such as in housekeeping genes), it will likely decrease in H2Bub1 occupancy and

244 gene expression between CMes and CP. In contrast, if $\mathrm{H} 2 \mathrm{Bub} 1$ is relatively even throughout the

245 gene body (such as in calcium signaling genes), it will instead maintain H2Bub1 occupancy and

246 gene expression (Fig. S4c, 2i). This implies that patterned H2Bub1 marks precede loss of the

247 mark and decreased expression, while evenly distributed H2Bub1 marks are retained and predict 248 continued expression.

250 Mutations in the RNF20-complex affect cardiomyocyte differentiation

Since H2Bub1 is selectively maintained on sarcomeric calcium signaling genes linked to

252 cardiomyopathy, along with the known link between ubiquitinase complex members ( $R N F 20$ and

$253(U B E 2 B)$ and $\mathrm{CHD}$, we next asked how defective H2Bub1 impacts CM development. To address

254 this, we first established iPSC lines with mutations affecting $R N F 20$, and evaluated how the

255 mutations impact the capacity for iPSCs to differentiate into CMs. In each of two independent

256 CRISPR experiments, we created one $R N F 20^{+/-}$iPSC line. The two independent iPSC lines have

257 different mutations, and are both predicted to be functionally haploinsufficient trans-

258 heterozygotes. For simplicity, we will be referring to these iPSC lines collectively as $R N F 20^{+/}$ 
259 (Fig. S5a, online methods). RNF20 knockdown was verified by western blotting for RNF20

260 (Fig. 4i, S6d).

261

$\mathrm{RNF}_{20} \mathrm{O}^{+-}$and wild-type iPSCs were then simultaneously differentiated into $\mathrm{CMs}^{38}$. At

262 iPSC, M, and CM stages (when the cells are relatively homogenous),we performed

263 immunofluorescence for markers of pluripotency (OCT4), M (Brachyury), CMes (NKX2.5), CP

264 (ISL1), and CM (cardiac troponin T, TNNT) ${ }^{38}$. At the iPSC stage, both wild-type and $R N F 20^{+/-}$

265 cells express OCT4, indicating that haploinsufficiency for RNF20 does not affect pluripotency

266 (Fig. S7a). At the M stage, again both wild-type and $R N F 20^{+/-}$cells express Brachyury, but only

267 wild-type cells lose their OCT4 expression, suggesting the $R N F 20^{+/-}$cells have a delay in exiting

268 pluripotency (Fig. S7b).

269 Wild-type CMs beat, no longer express Brachyury, and instead express NKX2.5, ISL1,

270 and TNNT. However, most cells in both $R N F 20^{+-}$lines continue to express Brachyury, and have

271 no NKX2.5 or TNNT expression. Interestingly, the $R N F 20^{+/}$cells do express ISL1, a pan-

272 cardiac marker, despite not expressing NKX2.5 (Fig. S7c). Since ISL1 is expressed in all CP

273 cells, this may indicate that $R N F 2 O^{+/-}$cells are in a less mature state ${ }^{54,55}$. Importantly, unlike

274 wild-type cells, most $R N F 20^{+/-}$cells fail to beat (even by day 20) (Fig. 3a, movie 1, movie 2,

275 movie 3, movie 4). In conclusion, the $\mathrm{RNF}_{2} \mathrm{O}^{+/-}$cell lines fail to differentiate into normal CMes,

276 and thus ultimately fail to make beating CMs.

277 To further investigate the possible role for the RNF20-complex on CM differentiation,

278 we also established iPSC lines with mutations affecting $U B E 2 B$, and evaluated how the

279 mutations impact the capacity for iPSCs to differentiate into CMs. In each of two independent

280 CRISPR experiments, we created one $U B E 2 B^{-/}$iPSC lines. Since UBE2A and UBE2B are more

281 than $95 \%$ identical at the protein level, these knockouts were validated by sequencing the cDNA 
282 (Fig. S8a, online methods) ${ }^{56}$. Mutant and wild-type iPSCs were then simultaneously

283 differentiated into $\mathrm{CMs}^{38}$. As with the $R N F 20^{+/-}$iPSCs, $U B E 2 B^{-/}$iPSCs mirror wild-type, but by

$284 \mathrm{M}$ stage, most $U B E 2 B^{-/}$cells gain Brachyury expression, while retaining OCT4 expression (Fig.

285 S7a, S7b).

In contrast to the $R N F 2 O^{+/}$cell lines, about a third of $U B E 2 B^{-/-}$cells $\left(U B E 2 B^{-/-} 125 / 72\right.$,

$287 U B E 2 B^{-/ 2} 24 / 69$ ) beat at the CM stage (Fig. 3a, movie 1, movie 5, movie 6, movie 7, movie 8).

288 Given this heterogeneity, we evaluated both beating (lactate selection) and non-beating (no

289 lactate selection) cells at a time corresponding to the wild-type CM stage (Online Methods).

290 The beating $U B E 2 B^{-/}$cells are mostly Brachyury negative, and NKX2.5, ISL1, and TNNT

291 positive, while the non-beating $U B E 2 B^{-/}$cells are mostly Brachyury and ISL1 positive, and

292 NKX2.5 and TNNT negative (Fig. S7c). These data indicate that $U B E 2 B^{-/}$iPSCs can

293 differentiate into beating CMs, but do so at a reduced efficiency compared to wild-type iPSCs.

294 Additionally, non-beating $U B E 2 B^{-/-}$cells are phenotypically equivalent to $R N F 2 O^{+-}$cells.

295 Collectively, these data demonstrate a requirement for the RNF20-complex in normal CM

296 differentiation.

297

298

RNF20-complex component dosage determines level of H2Bub1 deposition

299

To relate the dynamic nature of $\mathrm{H} 2 \mathrm{Bub} 1$ over CM differentiation and mouse heart

300 development to the ubiquitination complex (RNF20-RNF40-UBE2B and the scaffolding protein

301 WAC), we examined the expression of the complex components. To do this, we first asked how

302 the expression of the RNF20-complex changes over heart development in-vivo. Protein levels of

303 RNF20-complex members are dynamic between embryonic day 9.5 (e9.5) and adult mouse

304 hearts. RNF20 peaks at e11.5 and precipitously drops after birth, while RNF40 peaks at e14.5 
and also precipitously drops after birth. UBE2A/B increases up until adulthood. Importantly,

306 changes in these complex components affect H2Bub1 deposition; H2Bub1 is lowest at e9.5 and

307 highest in adult mice (Fig. S1e). These observations suggest that the ratio of the RNF20-complex

308 components may play an important role in H2Bub1 deposition efficiency.

309 We then determined the trajectory of RNF20-complex mRNA during CM differentiation

310 using RNA-seq data. $U B E 2 B$ and $R N F 40$ are relatively constant over CM differentiation, while

311 WAC reaches a maximum and $R N F 20$ reaches a minimum at the CP stage (Fig. 4a). Protein

312 levels do not directly correlate with mRNA; RNF20, RNF40, and UBE2A/B proteins all peak at

313 the CP stage, before falling to very low levels at the CM stage (Fig. 4b, S6a). The differences in

314 mRNA and protein levels could be secondary to persistence of long-lived protein from earlier

315 developmental time points, or from post-transcriptional regulation. In addition, UBE2B levels

316 reflect both UBE2B and UBE2A, which are $>95 \%$ identical at the protein level, and cannot be

317 discriminated by Western blotting ${ }^{56}$. The dynamic levels of RNF20-complex components and

318 lack of concordance of any individual complex component to the resulting H2Bub1 levels

319 suggest that deposition efficiency may depend on the ratio of the components, as it does in yeast.

320 Previous work demonstrated that a higher amount of Lge1 (corresponding to WAC) relative to

321 Bre1 (corresponding to RNF20/RNF40) leads to a better complex condensate, and is predicted to

322 more efficiently deposit $\mathrm{H} 2 \mathrm{Bub} 1^{16}$. Therefore, we would predict that at the CP stage, when WAC

323 is most highly expressed, H2Bub1 would be the highest, consistent with our observation (Fig.

324 2d, 4c, S3d). Our data both confirm and expand these findings to mammalian cells. In

325 conclusion, as the ratios of the complex components change, the total H2Bub1 changes in

326 response (Fig. $\mathbf{4 d})$. 
To test this further, we asked how global H2Bub1 deposition changes in our iPSC and

328 mouse RNF20-complex mutants. Analysis of the $R N F 20^{+/-}$cell lines was limited to the iPSC and

$329 \mathrm{M}$ stages, since they fail to differentiate further. As expected $R N F 20^{+/}$iPSCs have decreased

$330 R N F 20$ mRNA and protein, but also have upregulated $R N F 40 \mathrm{mRNA}$ and protein, and decreased

331 WAC, perhaps to attempt to correct the abnormal ratio (Fig. $\mathbf{4 h}, \mathbf{4 i}, \mathbf{S 6 d}, \mathbf{S 6 f}$ ). Interestingly, these

332 cells also have a paradoxical increase in total H2Bub1 (Fig. 4j, S6e). We propose that the

333 abnormal ratio of complex components may increase the efficiency of the complex, leading to

334 the paradoxical increase in the amount of H2Bub1 (Fig. 4k). We evaluated the genome-wide

335 H2Bub1 binding profile and gene expression in $R N F 20^{+/-}$iPSCs (S5b, Supplemental Data 3,

336 Supplemental Data 4). Consistent with the increased total H2Bub1 levels, H2Bub1 near gene

337 bodies is also increased and correlates with transcriptional changes (Fig. 4j, 5a, 5b, 5c, S6e).

338 DAVID GO enrichment analysis on genes with increased H2Bub1 occupancy and mRNA

339 expression indicate that many of these genes are involved with transcription, splicing, and/or

340 DNA and protein modifications (Fig. 5d). Since these classes of genes broadly affect

341 downstream transcription, haploinsufficiency for RNF20 likely leads to pleiotropic effects.

342 By M stage, RNF20 mRNA is restored to normal levels in $R N F 20^{+/-}$cells, but RNF20

343 protein remains decreased compared to wild-type (Fig. $4 \mathbf{h}, \mathbf{4 i}$, S6d, S6f). This suggests attempted

344 autoregulation of RNF20, since RNF20 itself has increased H2Bub1in both $R N F 20^{+/}$iPSC lines

345 compared to wild-type iPSCs (Supplemental Data 3). As in the $R N F 20^{+/-}$iPSCs, $W A C$ mRNA

346 is also decreased (Fig. 4h, S6f). The genome-wide H2Bub1 binding profile and gene expression

347 in $R N F 20^{+/-} \mathrm{M}$ cells indicate that $R N F 20$ haploinsufficiency causes pleiotropic responses upon

348 exiting pluripotency (Supplemental Data 3, Supplemental Data 4). There is a lack of genes

349 with significant differential H2Bub1 occupancy in $R N F 2 O^{+/}$cells compared to wild-type, even 
350 though there is an overall decrease in global H2Bub1 (Fig. S5c, S5d, Supplemental Data 3). By

351 the time the $R N F 2 O^{+/}$cells reach the M stage, their HBub1 levels deviate so far from normal

352 levels that there is catastrophic dysregulation of gene expression, which prevents the cells from

353 continuing to differentiate.

$354 U B E 2 B^{-/}$cell lines have reduced RNF20 and RNF40 levels at both iPSC and M stages,

355 possibly due to decreased $\mathrm{H} 2 \mathrm{Bub} 1$ on these genes, resulting in transcriptional downregulation

356 (Fig. 4e, S6b). As expected, loss of UBE2B results in decreased total H2Bub1 at the iPSC and M

357 stages (Fig. 4f, S6c). The small amount of residual H2Bub1 is likely retained in $U B E 2 B^{-/-}$cell

358 lines due to the presence of UBE2A, which has been shown to be partially redundant with

359 UBE2B with regard to $\mathrm{H} 2 \mathrm{Bub} 1^{14,56}$. Partial compensation of UBE2A by UBE2B is also

360 supported by the observation that $U b e 2 b^{-/-}$and $U b e 2 a^{-/-}$mice have abnormal fertility, but can

361 survive to adulthood ${ }^{57,58}$, while $R n f 20^{-/-}$mice are pre-implantation lethal.

362 Next we asked how H2Bub1 changes in e12.25 embryonic mouse hearts in response to

363 FHF-specific deletion of Rnf20 (Rnf2 $\left.0^{f l /-}:: N k x 2.5 \mathrm{Cre}^{+}\right)$. Both RNF20 and H2Bub1 are reduced in

$364 R n f 20^{f / /}: \because N k x 2.5 \mathrm{Cre}^{+}$mouse embryo hearts. The residual H2Bub1 and RNF20 is perhaps due to

365 the heterogeneity of the whole heart tissue, which includes both NKX2.5 (Rnf20-null) and

366 NKX2.5 (Rnf20-wild-type) cells (Fig. 4l, 4m, S6g).

367 Together, our data suggest that partial and complete loss of RNF20 differentially affect

368 H2Bub1. RNF20 haploinsufficiency raises the RNF20-complex efficiency, which results in

369 increased H2Bub1 (Fig. 4k). Complete loss of either RNF20 or UBE2B leads to a failure to form

370 a functional RNF20-complex, and reduction in total H2Bub1 (Fig. 4g, 4n). Thus, we propose

371 that dynamic changes in ratios of the RNF20-complex components over developmental time 
372 create a mechanism to precisely modulate H2Bub1 patterns and temporally regulate H2Bub1-

373 dependent gene transcription (Fig. 4d).

374

375 Total H2Bub1 reduction decreases sarcomeric calcium signaling gene expression in-vitro and in-vivo

In contrast to $R N F 2 O^{+/-}$cells, the $U B E 2 B^{-/-}$cells have decreased total $\mathrm{H} 2 \mathrm{Bub} 1$ and are 378 able to form beating CMs. To understand how reduced H2Bub1 could alter CM gene expression, 379 we performed RNA-seq analysis of beating $U B E 2 B^{-/} \mathrm{CMs}$, and compared them to time-matched 380 wild-type iPSC-derived CMs (Fig. 6a, S8b). Differential expression analysis identified 1393 381 downregulated and 1555 upregulated transcripts that are shared between both independent

$382 U B E 2 B^{-/}$cell lines compared to wild-type (Supplemental Data 6). To obtain a biological 383 context for the genes with decreased expression, we did DAVID GO enrichment analysis. About 384 half of the significant GO terms (37/76) are related to sarcomeric calcium genes, sarcomere 385 genes, and/or cardiomyopathy genes. Most notably, two genes with decreased expression, $386 C A C N A 1 C$ and $R Y R 2$, are also amongst the genes with selectively maintained H2Bub1 upon 387 wild-type transition from CMes to CP (Fig. 6c).

388 To determine if this in-vitro mechanism also functions in-vivo, we analyzed expression of 389 the embryonic sarcomeric calcium signaling genes (Cacnalc, Ryr2, Ncx, and Serca2a) at e11.5 390 in $R n f 20^{f l /+}: \because N k x 2.5 \mathrm{Cre}^{+}$and $R n f 20^{f l /}: \because N k x 2.5 \mathrm{Cre}^{+}$embryonic hearts, prior to any visible cardiac 391 defects (Fig. 6a). We observed that the $\operatorname{Rnf} 20^{f l-}: \because N k x 2.5 \mathrm{Cre}^{+}$have significantly lower Cacnalc 392 and Serca2a expression than $R n f 20^{f l /+}:: N k x 2.5 \mathrm{Cre}^{+}$siblings (Cacnalc $\mathrm{p}$-value $=0.03$, Serca2a p393 value $=0.001)($ Fig. 6b). Thus, RNF20-complex dependent H2Bub1 is necessary for normal 394 sarcomeric calcium gene expression in iPSC-derived CMs and in mice. 
Our results showing a decrease in cardiac sarcomeric gene expression in $U B E 2 B^{-/-} \mathrm{CMs}$,

397 along with their beating inefficiency, led us to evaluate the effect of reduced $\mathrm{H} 2 \mathrm{Bub} 1$ on cardiac

398 sarcomere structure in-vivo. Transmission electron microscopy revealed a missing H zone in

$399 \mathrm{e} 12.25 \mathrm{Rnf} 20^{f l /}:: N k x 2.5 \mathrm{Cre}^{+}$mouse sarcomeres (Fig. 3b). The $\mathrm{H}$ zone is the region of the

400 sarcomere that is devoid of actin filaments. At the center of the $\mathrm{H}$ zone is the $\mathrm{M}$ band, consisting

401 mostly of myomesin at e12.25, which functions to anchor the filaments to titin. Due to an elastic

402 domain in the middle of the embryonic splice variant of myomesin, this structure is not able to be

403 seen on TEM at this stage. An abnormal M band will lead to abnormal sarcomere organization,

404 as observed in the $\operatorname{Rnf} 20^{f l x /-}:: N k x 2.5 \mathrm{Cre}^{+}$mice $^{59}$. Interestingly, our RNA-seq data show that two

405 components of the M-band (Myomesin and Titin) and two proteins required for regulating their

406 alternative splicing ( $R B M 20$ and $R B M 24)$ have decreased expression in the $U B E 2 B^{-/} \mathrm{CMs}$

407 compared to the wild-type (median b value $=-1.54)^{60,61}$. This indicates that $\mathrm{H} 2 \mathrm{Bub} 1$ regulates $\mathrm{M}$

408 band proteins, which are required for normal sarcomeric structure and beating efficiency.

410 Accumulation of H2Bub1 near the center of tissue-specific genes correlates with enhanced

411 efficiency of transcriptional elongation

412 Strikingly, while loss of $U B E 2 B$ leads to decreased total H2Bub1 levels, ChIP-seq of

$413 \mathrm{CMs}$ comparing $\mathrm{H} 2 \mathrm{Bub} 1$ between wild-type and $U B E 2 B^{-/-}$cells demonstrates that gene-specific

414 H2Bub1 is only decreased in 8 Ensembl genes (Fig. 4f, S6c, S8c, Supplemental Data 5). To

415 identify differences in $\mathrm{H} 2 \mathrm{Bub} 1$ patterns between $U B E 2 B^{-/}$and wild-type CMs, we identified

416 calcium and/or sarcomeric genes with reduced mRNA in $U B E 2 B^{-/} \mathrm{CMs}$, along with calcium

417 signaling genes that selectively maintain H2Bub1 between CMes and CP. We then created 
418 metagenes by combining our selected genes ChIP-seq data and averaging the replicates,

419 allowing us to visualize common features of H2Bub1 for those gene sets (calcium genes $\mathrm{n}=28$,

420 sarcomere genes $n=70$, Supplemental Data 5). Wild-type cells have an accumulation of

421 H2Bub1 near the center of the metagenes, but this accumulation is either reduced or completely

422 absent in $U B E 2 B^{-/-}$cells $($Fig. 7a, 7b). To validate that this accumulation is not a technical

423 artifact, we generated 30 sets of "random" quantity and sized-matched gene sets for both the

424 calcium and sarcomeric genes. To control for the expression differences in these genes, we chose

425 an even distribution of genes sets that were upregulated, downregulated, or unchanged between

426 both mutants compared to wild-type. While accumulation is present near the center of some of

427 these metagenes, none of them show differential H2Bub1 occupancy at the accumulation region

428 between mutant and wild-type cells. We provide one representative graph (Fig. 7c). We next

429 determined whether this phenomenon is true for all genes, or just tissue-specific long genes by

430 producing metagene plots for all short genes (less than $100 \mathrm{bp}$ ) and all long genes (greater than

$43110 \mathrm{~Kb}$ ). We conclude that H2Bub1 accumulation near the center of genes is present in all long

432 genes, but UBE2B-dependent accumulation is unique to tissue-specific long genes (Fig. S8d,

433 S8e). Therefore, the H2Bub1 accumulation near the center of the gene is specific only to certain

434 gene classes, including calcium and sarcomeric genes.

435 Since the RNF20-complex is known to be involved in transcriptional elongation and

$436 \mathrm{H} 2 \mathrm{Bub1}$ is found on long tissue-specific genes during left-right patterning of the heart, we

437 hypothesized that the accumulation of H2Bub1 near the center of long genes may support their

438 transcriptional elongation (median length of all genes is $31 \mathrm{~Kb}$, compared to 456 and $69 \mathrm{~Kb}$ for

439 calcium signaling and sarcomeric genes, respectively). To test this hypothesis, we asked whether

440 there was loss of the 3 end o transcripts in $U B E 2 B^{-/-} \mathrm{CMs}$ compared to wild-type. When 
441 comparing the ratio of mutant to wild-type RNA in both the calcium and sarcomeric gene sets,

442 transcripts are less abundant in $U B E 2 B^{-/}$cells in the most 320 o the gene indicating

443 inefficient transcription elongation in the mutants; example transcript traces for $C A C N A 1 C$ and

$444 R Y R 2$ are shown (Fig. 7d, 7e, $7 \mathbf{g})$. To validate this conclusion, we repeated the same analysis on 445 our "random" gene sets hich onl had transcriptional e icienc drop-off in the last 5-10\% of

446 the 3 end. We provide one representative graph (Fig. 7f). Thus, these data support the

447 conclusion that accumulation of $\mathrm{H} 2 \mathrm{Bub1}$ near the center of calcium and sarcomeric genes is

448 UBE2B dependent and correlates with enhanced transcription elongation efficiency.

449 We next asked if this H2Bub1 accumulation is unique to CMs. To address this, we used 450 previously published H2Bub1-ChIP-seq data of wild-type mESCs and MEFs ${ }^{33}$. We determined

451 the MEF-specific genes by identifying differentially expressed genes between MEFs and

452 mESCs; they are enriched in adhesion $(n=143)$, extra-cellular matrix $(\mathrm{ECM})(\mathrm{n}=130)$, and ions

$453(\mathrm{n}=160)$ GO terms (Supplemental Data 5). Similar to the CM tissue-specific genes, these

454 genes are also long (median length is $43 \mathrm{~Kb}$ for adhesion, $48 \mathrm{~Kb}$ for ECM, and $50 \mathrm{~Kb}$ for ions).

455 The metagene plots generated for adhesion and ECM show an accumulation of H2Bub1 near the

456 center of the gene, similar to the calcium and sarcomeric genes in CMs. Additionally, this

457 accumulation was higher in the tissue with increased expression of the differentially expressed

458 genes (MEFs), as expected (Fig. 7h, 7i). As in CMs, all of the quantity and size-matched

459 "random" genes sets generated show no accumulation of H2Bub1 near the center of the gene, as

460 shown in one representative graph (Fig. 7j). The CM and MEF data collectively suggest that

461 accumulation of H2Bub1 near the center of tissue-specific long genes is a general mechanism for

462 regulation of transcriptional efficiency. In CMs, central H2Bub1 accumulation and efficient 
463 transcription of sarcomeric and calcium signaling genes depend on UBE2B, providing a

464 mechanism to explain the cardiac phenotype in the $R n f 20^{f l f l}:: N k x 2.5$ Cre mouse model

465

466 Discussion

467 Together, our data support a requirement for tight control of H2Bub1 levels in cardiac

468 development. Increased total H2Bub1 leads to failure to form CMs, while decreased total

$469 \mathrm{H} 2 \mathrm{Bub1}$ reduces the efficiency of CM differentiation in-vitro, and leads to reduced expression of

470 calcium signaling genes and structural abnormalities of the cardiac sarcomere, including a

471 deficient H zone, in-vivo (Fig. 8a). H2Bub1 is highly dynamic during CM differentiation: early,

472 the mark is increased and then decreased on housekeeping genes, and later the mark is

473 selectively maintained on calcium signaling genes, while it shifts from euchromatic to

474 heterochromatic regions (Fig. 8b). Finally, we show that the shape of the H2Bub1 mark changes

475 between wild-type and $\mathrm{UBE}^{-/-} \mathrm{BMs}$. The profile of $\mathrm{H} 2 \mathrm{Bub} 1$ on short genes is as previously

476 reported, with coverage over the entire gene- od higher at the 5 end than the 3 end o the

477 gene ${ }^{17}$. Notably, long genes have a vastly different profile: there is tissue-specific UBE2B-

478 dependent $\mathrm{H} 2 \mathrm{Bub} 1$ accumulation near the center of the gene, which correlates with efficient

479 transcriptional elongation (Fig. 8c).

480 H2Bub1 is an enigmatic chromatin mark localizing to the entire gene body, instead of

481 discreet promoter and enhancer regions. At the onset of CM differentiation, we highlight an

482 increase in both the total and gene-specific H2Bub1, as seen previously in neuronal cells and

483 keratinocytes $^{21}$. Further, our data support a model where tissue-specific transcriptional effects are

484 determined by the retention, instead of deposition, of $\mathrm{H} 2 \mathrm{Bub} 1$ on tissue-specific genes. $\mathrm{H} 2 \mathrm{Bub} 1$

485 has been linked to transcriptional elongation, but how it affects transcriptional elongation is 
486 unclear $^{13,14,23,30,32,33,62}$. We observed accumulation of H2Bub1 near the center of a subset of long

487 genes. Interestingly, it was previously determined that RNA Pol II exhibits a similar

488 accumulation at the center of all expressed genes ${ }^{63}$. Since H2Bub1 co-localizes with RNA Pol II,

489 the published RNA Pol II distribution is in concordance with the H2Bub1 distribution ${ }^{30}$. We

490 propose that $\mathrm{H} 2 \mathrm{Bub} 1$ enhances transcriptional elongation of long tissue-specific genes. This

491 conclusion is supported by previous work finding that long genes are more likely to depend on

492 RNF20 to be induced upon differentiation into neuronal cells ${ }^{21}$. Further evidence is the presence

493 of $\mathrm{H} 2 \mathrm{Bub} 1$ on cilia genes (which have an average length of $73 \mathrm{~Kb}$ ) in the multi-ciliated cells of

494 oviducts (which require expression of long motile cilia genes), but not in the liver cells, which do

495 not $^{18}$. Combined with our current data analyzing CMs and MEFs, we propose that targeted

$496 \mathrm{H} 2 \mathrm{Bub} 1$ accumulation is a more general mechanism regulating tissue-specific transcriptional

497 elongation efficiency on long genes.

498 Ratios of RNF20-complex components affect the efficiency of H2Bub1 deposition. We

499 show that if Rnf2O is deleted, total H2Bub1 decreases; if RNF2O is haploinsufficient, total

$500 \mathrm{H} 2 \mathrm{Bub} 1$ paradoxically increases; if $U B E 2 B$ is deleted, total $\mathrm{H} 2 \mathrm{Bub} 1$ decreases. The varying

501 levels of H2Bub1 resulting from altered levels of distinct RNF20-complex components support

502 an existing hypothesis, whereby the exact stoichiometric ratios of the complex components affect

503 its efficiency ${ }^{16}$. Thus, missing a component will yield the expected results of no H2Bub1, but

504 having less of a component will affect the ratios of the complex components, altering the

505 efficiency. We suggest that iPSCs normally maintain complex components in a less efficient

506 ratio to constrain the level of $\mathrm{H} 2 \mathrm{Bub} 1$ to maintain pluripotency.

507 We propose that heart development is exquisitely sensitive to H2Bub1 dosage. The

508 difference in phenotype between the mice with RNF20 deleted in the FHF and SHF is striking, 
509 suggesting that the SHF-derived tissues (mostly endocardium and epicardium) are less sensitive

510 to altered H2Bub1 dosage than the FHF-derived tissues (mostly cardiomyocytes) ${ }^{64,65}$. Since

511 RNF20 is required to exit pluripotency, the presence of H2Bub1 on a subset of genes is crucial

512 for this process to occur. Once the cells exit pluripotency, these marks become restricted to

513 heart-specific genes. If there is an abnormal amount of H2Bub1 upon exiting pluripotency, the

514 cells are unable to fully differentiate. Our data indicate that RNF20-complex-dependent H2Bub1

515 is necessary for normal cardiac development through regulating the transcriptional elongation

516 efficiency of cardiac calcium signaling and sarcomeric genes during CM differentiation and

517 development.

518 The question remains how H2Bub1 affects development of cardiac structure, since both

519 human patients with mutations affecting H2Bub1 and mouse embryos with cardiac-specific

520 deletion of $R n f 20$ have structural heart defects. A possible link to the observed structural heart

521 defects in mice is that altered expression of calcium signaling genes and abnormal sarcomeric

522 structure observed in $\operatorname{Rnf} 20^{\mathrm{fl} /-}:: N k x 2.5 \mathrm{Cre}^{+}$mouse embryos led to defective cardiac function

523 during embryonic development, and that the resulting hemodynamic derangement affects

524 structural cardiac morphogenesis. Extensive evidence supports interdependence between

525 embryonic hemodynamics and valve development, cardiac trabeculation, myocardial

526 proliferation and formation of the epicardium (reviewed in ${ }^{66}$ ). Genomic studies of human CHD

527 patients are beginning to provide further evidence of an overlap between genes classically linked

528 to cardiomyopathy, and patients presenting with structural CHD. For example, dominant

529 mutations affecting myosin heavy chain 6 (MYH6) are associated with cardiomyopathy and atrial

530 septal defects ${ }^{67,68}$, while recessive mutations in $M Y H 6$ are found in $11 \%$ of patients with Shone

531 syndrome, characterized by valve defects and multiple levels of left ventricular obstruction ${ }^{3}$. The 
532 essential role of $\mathrm{H} 2 \mathrm{Bub} 1$ in $\mathrm{CM}$ development and cardiac morphogenesis in mouse and human

533 provide further support for genetic overlap between cardiac structure development and

534 myocardial function, and suggest that a subset of patients with structural heart defects caused by

535 genetic defects affecting cardiomyocytes may be more vulnerable to myocardial dysfunction.

536 Although there are likely variations in the absolute H2Bub1 levels required for normal iPSC-

537 derived CM development, mouse embryo, and human heart development, our observations in

538 iPSC-derived CMs and mouse embryos indicate a shared requirement for precise control of

539 H2Bub1 in the heart.

540

541 Acknowledgements

542 We thank the Center for Cellular and Molecular Imaging at Yale for transmission electron

543 microscopy and the Developmental Histology Tissue Services at Yale for adult mouse heart

544 histology. We would like to thank Dr. Jon Seidman and Dr. Christine Seidman for their

545 assistance with the human iPSC work. We would also like to thank Dr. Yibing Qyang and Dr.

546 William Pu for sharing mouse lines with us. This work was supported by NSFGRFP to SB, and

547 R35HL145249 and 2UO1HL098162 to MB.

548

\section{Author Contributions}

$550 \mathrm{SB}$ and $\mathrm{MB}$ conceived and designed the experiments. SB, JD, KB, IBB, LK, and MB performed

551 the experiments in mouse. SB and LW performed the experiments in the iPSCs. SB performed

552 the bioinformatics analysis of the ChIP-seq and RNA-seq experiments, and SC provided

553 conceptual advice on the analysis. SB and MB wrote the manuscript, which was read and

554 approved by all of the authors. 


\section{References}

556 1. Pierpont, M.E. et al. Genetic Basis for Congenital Heart Disease: Revisited: A Scientific Statement From the American Heart Association. Circulation 138, e653-e711 (2018).

2. Homsy, J. et al. De novo mutations in congenital heart disease with neurodevelopmental and other congenital anomalies. Science 350, 1262-6 (2015).

3. Jin, S.C. et al. Contribution of rare inherited and de novo variants in 2,871 congenital heart disease probands. Nat Genet 49, 1593-1601 (2017).

4. Zaidi, S. et al. De novo mutations in histone-modifying genes in congenital heart disease. Nature 498, 220-3 (2013).

5. Ang, S.Y. et al. KMT2D regulates specific programs in heart development via histone $\mathrm{H} 3$ lysine 4 di-methylation. Development 143, 810-21 (2016).

6. Jones, B. et al. The histone H3K79 methyltransferase Dot1L is essential for mammalian development and heterochromatin structure. PLoS Genet 4, e1000190 (2008).

7. Montgomery, R.L. et al. Histone deacetylases 1 and 2 redundantly regulate cardiac morphogenesis, growth, and contractility. Genes Dev 21, 1790-802 (2007).

8. Van Laarhoven, P.M. et al. Kabuki syndrome genes KMT2D and KDM6A: functional analyses demonstrate critical roles in craniofacial, heart and brain development. Hum Mol Genet 24, 4443-53 (2015).

9. Wamstad, J.A. et al. Dynamic and coordinated epigenetic regulation of developmental transitions in the cardiac lineage. Cell 151, 206-20 (2012).

10. Robzyk, K., Recht, J. \& Osley, M.A. Rad6-dependent ubiquitination of histone H2B in yeast. Science 287, 501-4 (2000).

11. Wood, A. et al. Bre1, an E3 ubiquitin ligase required for recruitment and substrate selection of Rad6 at a promoter. Mol Cell 11, 267-74 (2003).

12. Kim, J., An, Y.K., Park, S. \& Lee, J.S. Bre1 mediates the ubiquitination of histone H2B by regulating Lge1 stability. FEBS Lett 592, 1565-1574 (2018).

13. Kim, J., Hake, S.B. \& Roeder, R.G. The human homolog of yeast BRE1 functions as a transcriptional coactivator through direct activator interactions. Mol Cell 20, 759-70 (2005).

14. Kim, J. et al. RAD6-Mediated transcription-coupled H2B ubiquitylation directly stimulates H3K4 methylation in human cells. Cell 137, 459-71 (2009).

15. Zhang, F. \& Yu, X. WAC, a functional partner of RNF20/40, regulates histone H2B ubiquitination and gene transcription. Mol Cell 41, 384-97 (2011).

16. Gallego, L.D. et al. Phase separation directs ubiquitination of gene-body nucleosomes. Nature 579, 592-597 (2020).

17. Jung, I. et al. $\mathrm{H} 2 \mathrm{~B}$ monoubiquitylation is a 5'-enriched active transcription mark and correlates with exon-intron structure in human cells. Genome Res 22, 1026-35 (2012).

18. Robson, A. et al. Histone H2B monoubiquitination regulates heart development via epigenetic control of cilia motility. Proc Natl Acad Sci U S A 116, 14049-14054 (2019).

19. VanDusen, N.J. et al. Massively parallel in vivo CRISPR screening identifies RNF20/40 as epigenetic regulators of cardiomyocyte maturation. Nat Commun 12, 4442 (2021).

20. Chen, S., Li, J., Wang, D.L. \& Sun, F.L. Histone H2B lysine 120 monoubiquitination is required for embryonic stem cell differentiation. Cell Res 22, 1402-5 (2012). 
21. Fuchs, G. et al. RNF20 and USP44 regulate stem cell differentiation by modulating $\mathrm{H} 2 \mathrm{~B}$ monoubiquitylation. Mol Cell 46, 662-73 (2012).

22. Karpiuk, O. et al. The histone H2B monoubiquitination regulatory pathway is required for differentiation of multipotent stem cells. Mol Cell 46, 705-13 (2012).

23. Shema, E., Kim, J., Roeder, R.G. \& Oren, M. RNF20 inhibits TFIIS-facilitated transcriptional elongation to suppress pro-oncogenic gene expression. Mol Cell 42, 47788 (2011).

24. Shema, E. et al. The histone H2B-specific ubiquitin ligase RNF20/hBRE1 acts as a putative tumor suppressor through selective regulation of gene expression. Genes Dev 22, 2664-76 (2008).

25. Tarcic, O. et al. RNF20 Links Histone H2B Ubiquitylation with Inflammation and Inflammation-Associated Cancer. Cell Rep 14, 1462-76 (2016).

26. Wang, E. et al. Histone H2B ubiquitin ligase RNF2O is required for MLL-rearranged leukemia. Proc Natl Acad Sci U S A 110, 3901-6 (2013).

27. Lee, J.S. et al. Histone crosstalk between $\mathrm{H} 2 \mathrm{~B}$ monoubiquitination and $\mathrm{H} 3$ methylation mediated by COMPASS. Cell 131, 1084-96 (2007).

28. Xie, W. et al. RNF40 regulates gene expression in an epigenetic context-dependent manner. Genome Biol 18, 32 (2017).

29. Henry, K.W. et al. Transcriptional activation via sequential histone $\mathrm{H} 2 \mathrm{~B}$ ubiquitylation and deubiquitylation, mediated by SAGA-associated Ubp8. Genes Dev 17, 2648-63 (2003).

30. Fuchs, G., Hollander, D., Voichek, Y., Ast, G. \& Oren, M. Cotranscriptional histone H2B monoubiquitylation is tightly coupled with RNA polymerase II elongation rate. Genome Res 24, 1572-83 (2014).

31. Kim, J. \& Roeder, R.G. Direct Bre1-Paf1 complex interactions and RING fingerindependent Bre1-Rad6 interactions mediate histone $\mathrm{H} 2 \mathrm{~B}$ ubiquitylation in yeast. J Biol Chem 284, 20582-92 (2009).

32. Pavri, R. et al. Histone H2B monoubiquitination functions cooperatively with FACT to regulate elongation by RNA polymerase II. Cell 125, 703-17 (2006).

33. Wang, F. et al. Histone H2Bub1 deubiquitylation is essential for mouse development, but does not regulate global RNA polymerase II transcription. Cell Death Differ (2021).

34. Valenzuela, D.M. et al. High-throughput engineering of the mouse genome coupled with high-resolution expression analysis. Nat Biotechnol 21, 652-9 (2003).

35. $\mathrm{Xu}, \mathrm{Z}$. et al. H2B ubiquitination regulates meiotic recombination by promoting chromatin relaxation. Nucleic Acids Res 44, 9681-9697 (2016).

36. Moses, K.A., DeMayo, F., Braun, R.M., Reecy, J.L. \& Schwartz, R.J. Embryonic expression of an Nkx2-5/Cre gene using ROSA26 reporter mice. Genesis 31, 176-80 (2001).

37. Pfaff, S.L., Mendelsohn, M., Stewart, C.L., Edlund, T. \& Jessell, T.M. Requirement for LIM homeobox gene Isl1 in motor neuron generation reveals a motor neuron-dependent step in interneuron differentiation. Cell 84, 309-20 (1996).

38. Lian, X. et al. Directed cardiomyocyte differentiation from human pluripotent stem cells by modulating Wnt/beta-catenin signaling under fully defined conditions. Nat Protoc $\mathbf{8}$, 162-75 (2013). 
641 39. Bertero, A. et al. Dynamics of genome reorganization during human cardiogenesis reveal an RBM20-dependent splicing factory. Nat Commun 10, 1538 (2019).

643 40. Inoue, F. et al. A systematic comparison reveals substantial differences in chromosomal versus episomal encoding of enhancer activity. Genome Res 27, 38-52 (2017).

41. Kazachenka, A. et al. Identification, Characterization, and Heritability of Murine Metastable Epialleles: Implications for Non-genetic Inheritance. Cell 175, 1259-1271 e13 (2018).

42. Mei, H. et al. H2AK119ub1 guides maternal inheritance and zygotic deposition of H3K27me3 in mouse embryos. Nat Genet 53, 539-550 (2021).

43. Tang, Y., Tian, X., Wang, R., Fill, M. \& Chen, S.R. Abnormal termination of Ca2+ release is a common defect of RyR2 mutations associated with cardiomyopathies. Circ Res 110, 968-77 (2012).

44. Knight, W.E. et al. PDE1C deficiency antagonizes pathological cardiac remodeling and dysfunction. Proc Natl Acad Sci U S A 113, E7116-E7125 (2016).

45. Wang, X. et al. Generation and phenotypic characterization of Pdela mutant mice. PLoS One 12, e0181087 (2017).

46. Wang, L. et al. Dual LQT1 and HCM phenotypes associated with tetrad heterozygous mutations in KCNQ1, MYH7, MYLK2, and TMEM70 genes in a three-generation Chinese family. Europace 18, 602-9 (2016).

47. Vasti, C. \& Hertig, C.M. Neuregulin-1/erbB activities with focus on the susceptibility of the heart to anthracyclines. World J Cardiol 6, 653-62 (2014).

48. Xu, H. et al. A Genome-Wide Association Study of Idiopathic Dilated Cardiomyopathy in African Americans. J Pers Med 8(2018).

49. Boczek, N.J. et al. Identification and Functional Characterization of a Novel CACNA1CMediated Cardiac Disorder Characterized by Prolonged QT Intervals With Hypertrophic Cardiomyopathy, Congenital Heart Defects, and Sudden Cardiac Death. Circ Arrhythm Electrophysiol 8, 1122-32 (2015).

50. Kepenek, E.S. et al. Differential expression of genes participating in cardiomyocyte electrophysiological remodeling via membrane ionic mechanisms and $\mathrm{Ca}(2+)$-handling in human heart failure. Mol Cell Biochem 463, 33-44 (2020).

51. Mazzarotto, F. et al. Systematic large-scale assessment of the genetic architecture of left ventricular noncompaction reveals diverse etiologies. Genet Med 23, 856-864 (2021).

52. Vasilescu, C. et al. Genetic Basis of Severe Childhood-Onset Cardiomyopathies. J Am Coll Cardiol 72, 2324-2338 (2018).

53. Zhang, L. \& Zhang, S. Learning common and specific patterns from data of multiple interrelated biological scenarios with matrix factorization. Nucleic Acids Res 47, 66066617 (2019).

54. Prall, O.W. et al. An Nkx2-5/Bmp2/Smad1 negative feedback loop controls heart progenitor specification and proliferation. Cell 128, 947-59 (2007).

55. Dorn, T. et al. Direct nkx2-5 transcriptional repression of isl1 controls cardiomyocyte subtype identity. Stem Cells 33, 1113-29 (2015).

56. Sarcevic, B., Mawson, A., Baker, R.T. \& Sutherland, R.L. Regulation of the ubiquitinconjugating enzyme hHR6A by CDK-mediated phosphorylation. EMBO J 21, 2009-18 (2002). 
57. Roest, H.P. et al. The ubiquitin-conjugating DNA repair enzyme HR6A is a maternal factor essential for early embryonic development in mice. Mol Cell Biol 24, 5485-95 (2004).

58. Roest, H.P. et al. Inactivation of the HR6B ubiquitin-conjugating DNA repair enzyme in mice causes male sterility associated with chromatin modification. Cell 86, 799-810 (1996).

59. Lange, S., Pinotsis, N., Agarkova, I. \& Ehler, E. The M-band: The underestimated part of the sarcomere. Biochim Biophys Acta Mol Cell Res 1867, 118440 (2020).

60. Yang, J. et al. RBM24 is a major regulator of muscle-specific alternative splicing. Dev Cell 31, 87-99 (2014).

61. Schneider, J.W. et al. Dysregulated ribonucleoprotein granules promote cardiomyopathy in RBM20 gene-edited pigs. Nat Med 26, 1788-1800 (2020).

62. Sadeghi, L., Siggens, L., Svensson, J.P. \& Ekwall, K. Centromeric histone H2B monoubiquitination promotes noncoding transcription and chromatin integrity. Nat Struct Mol Biol 21, 236-43 (2014).

63. Young, M.D. et al. ChIP-seq analysis reveals distinct H3K27me3 profiles that correlate with transcriptional activity. Nucleic Acids Res 39, 7415-27 (2011).

64. Liang, X. et al. HCN4 dynamically marks the first heart field and conduction system precursors. Circ Res 113, 399-407 (2013).

65. Spater, D. et al. A HCN4+ cardiomyogenic progenitor derived from the first heart field and human pluripotent stem cells. Nat Cell Biol 15, 1098-106 (2013).

66. Andres-Delgado, L. \& Mercader, N. Interplay between cardiac function and heart development. Biochim Biophys Acta 1863, 1707-16 (2016).

67. Ching, Y.H. et al. Mutation in myosin heavy chain 6 causes atrial septal defect. Nat Genet 37, 423-8 (2005).

68. Hershberger, R.E. et al. Coding sequence rare variants identified in MYBPC3, MYH6, TPM1, TNNC1, and TNNI3 from 312 patients with familial or idiopathic dilated cardiomyopathy. Circ Cardiovasc Genet 3, 155-61 (2010). 
Figure 1

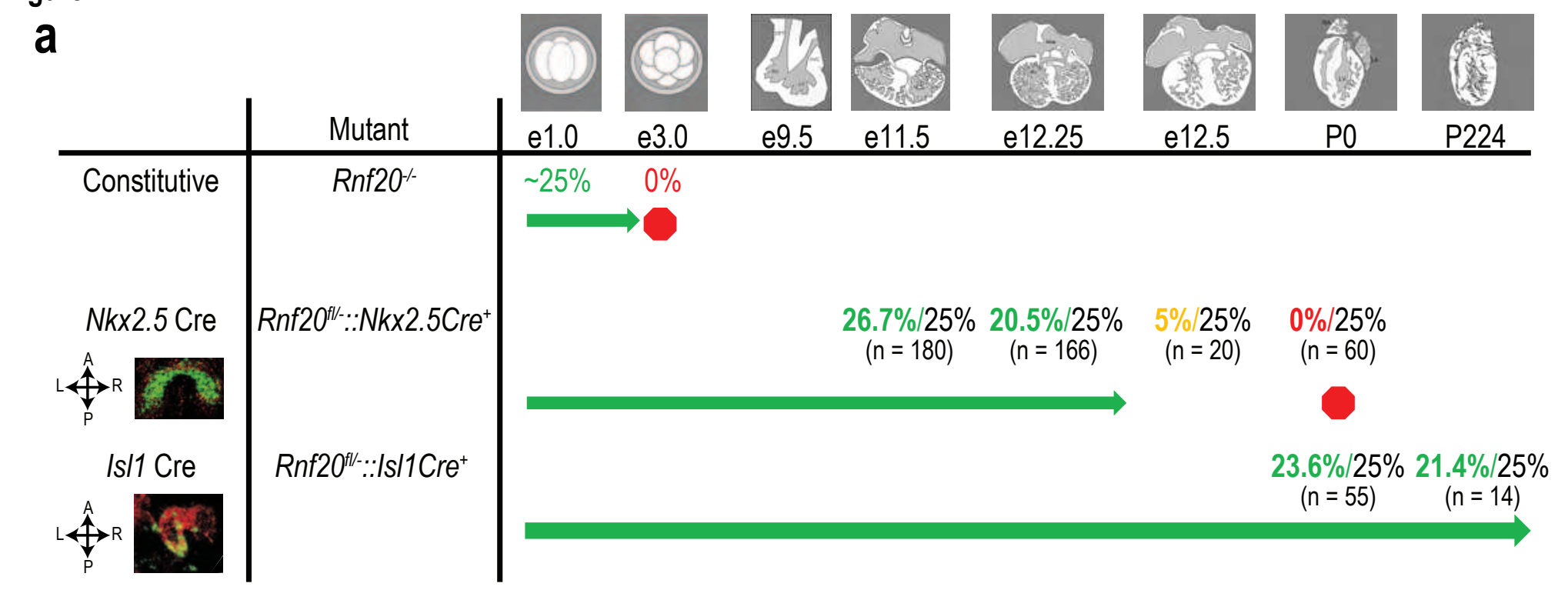

b
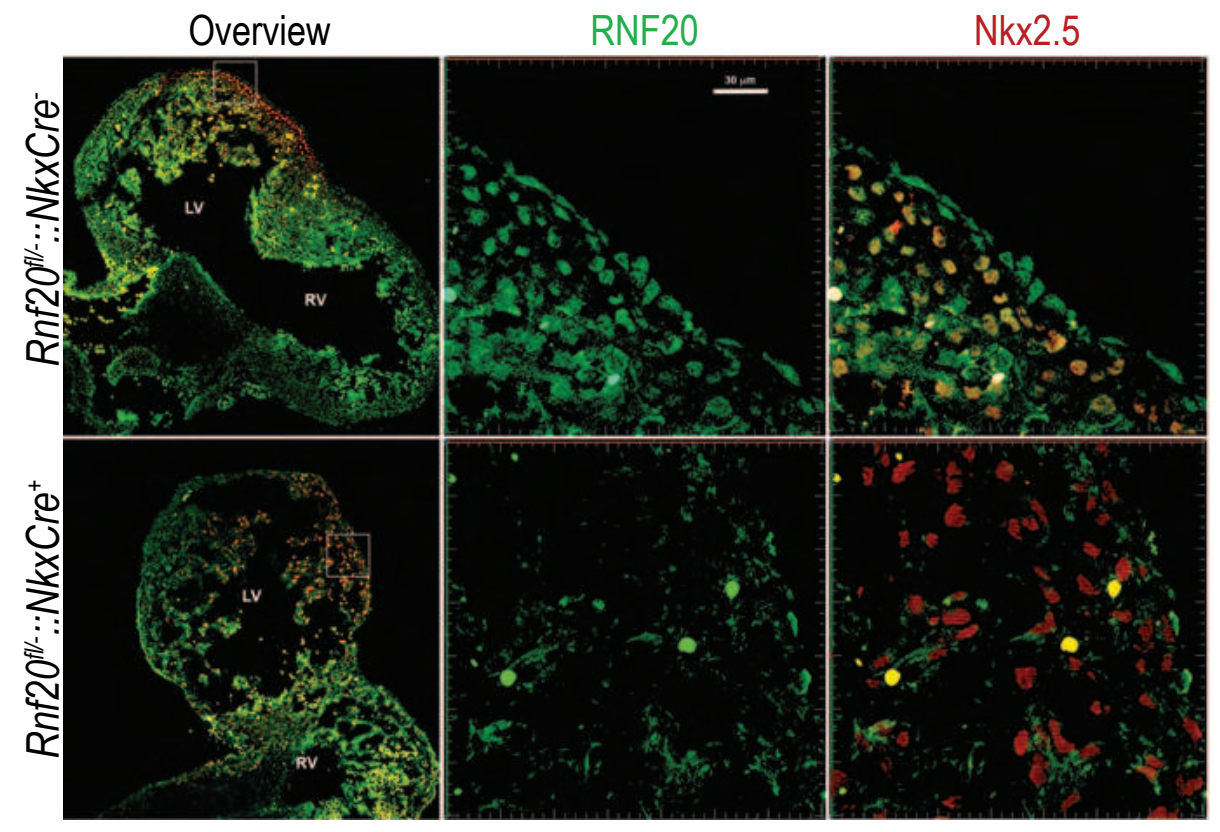

C
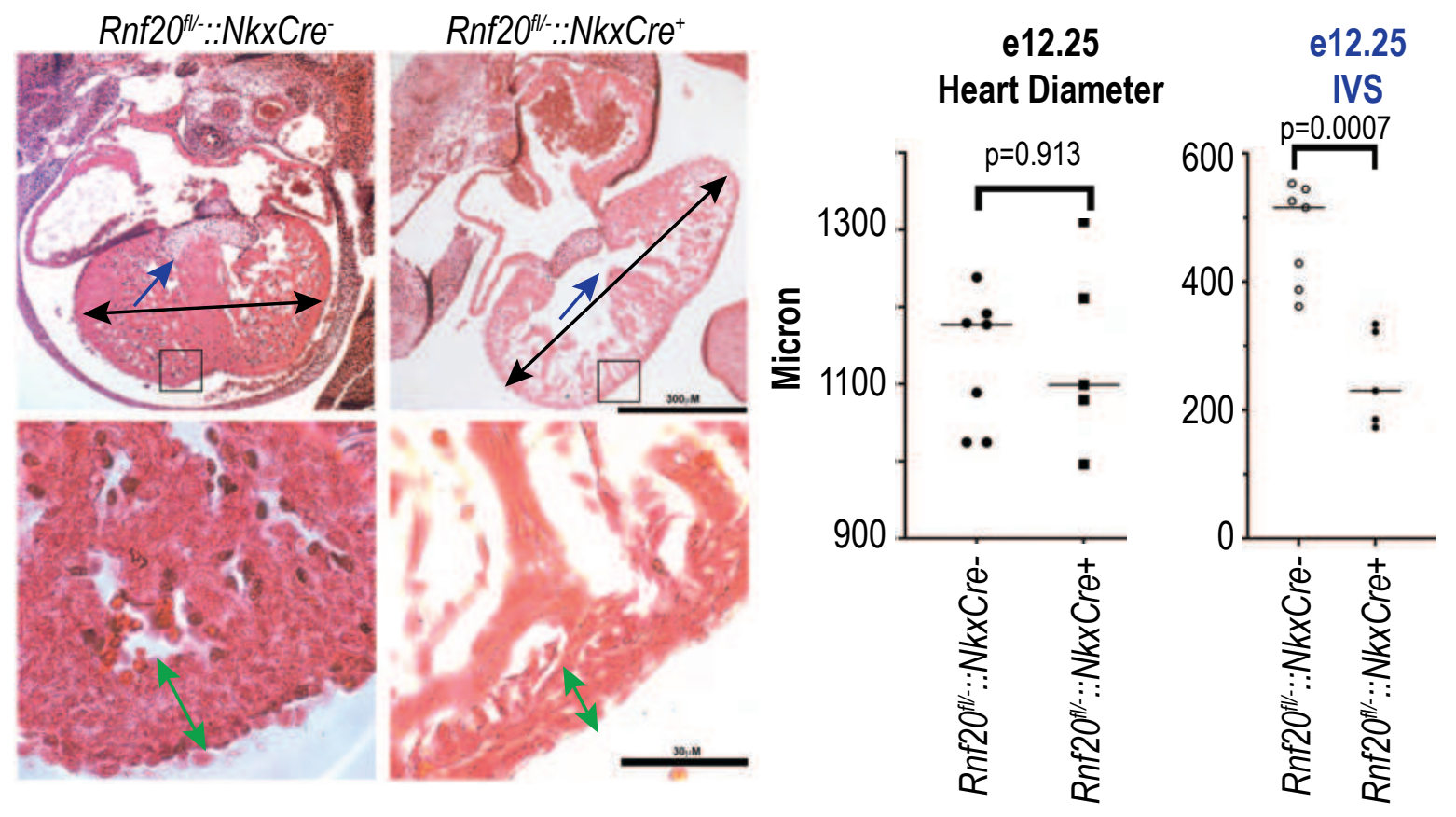

e12.25

Compact Myocardium

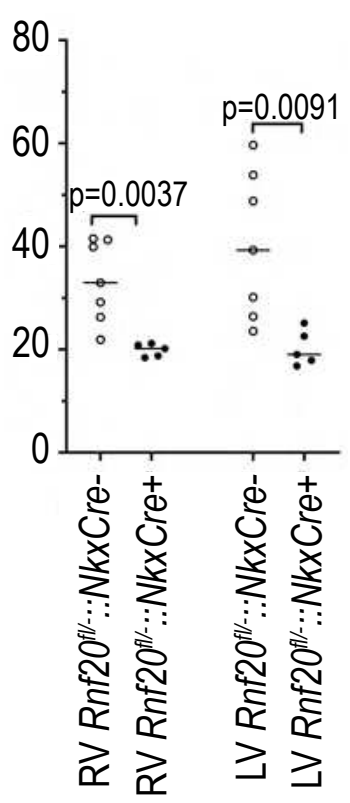




\section{Figure 1: $\operatorname{Rnf20}$ is required for heart development}

717 a) Rnf20 mutant mouse survival chart for constitutive nulls, $R n f 20^{\mathrm{fl} x /-}:: N k x 2.5 \mathrm{Cre}^{+}$, and $R n f 2 O^{\mathrm{flx} /-}:: I s l 1 \mathrm{Cre}^{+}$. Colored percentages indicate the observed percentage of null or

719 conditionally null mice (green indicates mendelian ratios, yellow indicates deviation from

720 mendelian ratios, and red indicates no mice). Black percentages indicate the predicted

721 percentages. The sample size is listed below each percentage. See Figure S2 for more details

722 on these crosses. Images were taken of the cross between a $N k x 2.5$-cre positive (e8.5 (cardiac

723 crescent), signal in cardiac crescent) or Isll-cre positive mouse (e9.0 (heart tube), signal in

724 outflow tract and atria) and a ROSA ${ }^{\mathrm{mt} / \mathrm{mg}}$ mouse to illustrate distribution of Cre-positive cells.

725 Drawings indicate the expected morphology at each stage mouse heart development. OFT

726 outflow tract, $\mathrm{RV}$ right ventricle, $\mathrm{LV}$ left ventricle, AVC atrioventricular canal, ECC

727 endocardial cushions, RA right atrium, LA left atrium, A anterior, $\mathrm{P}$ posterior, L

728 left, R - right.

729 b) Immunofluorescent staining for NKX2.5 and RNF20 in e11.5 wild-type (Rnf $20^{\mathrm{fl} / /}$

$\left.730 \quad:: N k x 2.5 \mathrm{Cre}^{-}\right)$and mutant $\left(\operatorname{Rnf} 20^{\mathrm{fl} /-}:: N k x 2.5 \mathrm{Cre}^{+}\right)$mouse hearts.

731 c) Example hematoxylin and eosin stained e12.25 wild-type (Rnf $\left.20^{\mathrm{fl} /-}:: N k x 2.5 \mathrm{Cre}^{-}\right)$and mutant

$732 \quad\left(\operatorname{Rnf} 20^{\mathrm{fl} /-}:: N k x 2.5 \mathrm{Cre}^{+}\right)$mouse. Quantifications of interventricular septum length, heart

733 diameter, thickness of right ventricle compact myocardium, and thickness of left ventricle

734 compact myocardium are displayed as individual data points with a line representing the

735 median ( $\mathrm{n}=7$ wild-type and 5 mutant hearts). Unpaired 2-tailed, heteroscedastic t-test. 


\section{Figure 2}

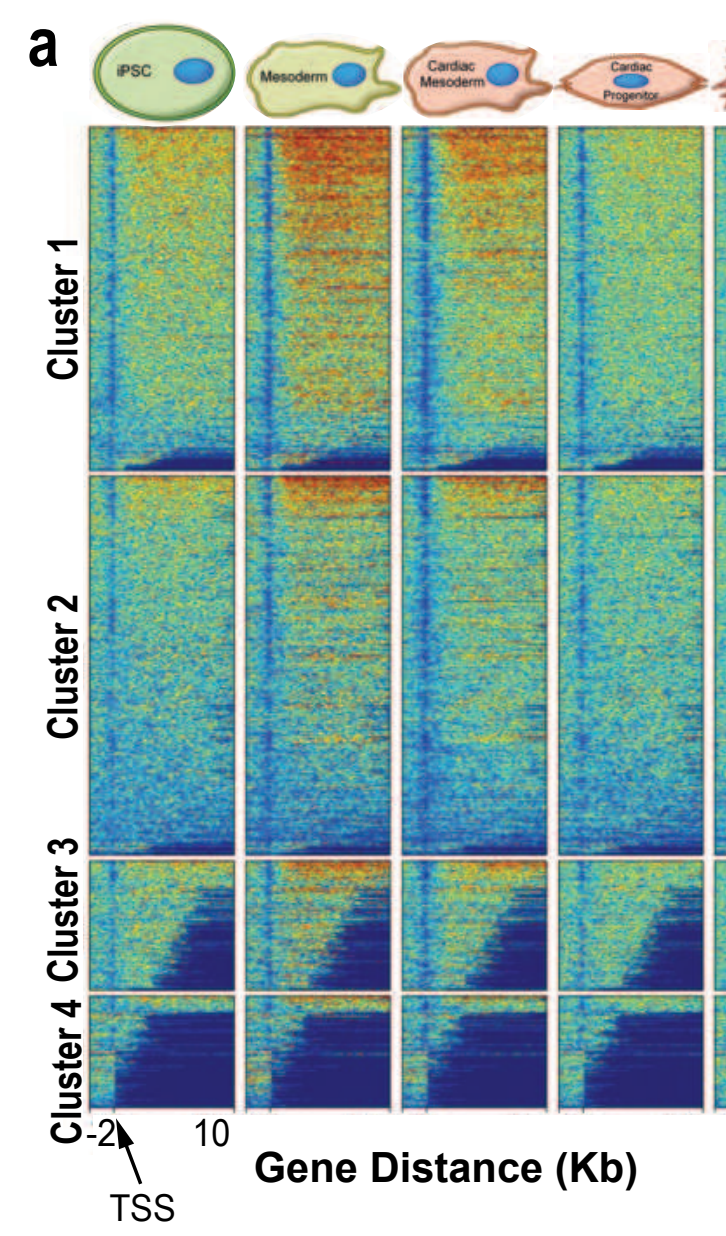

g
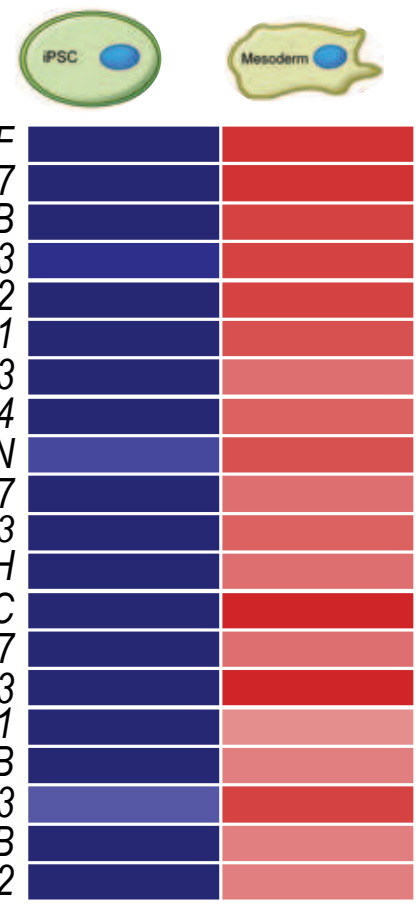

MTDH

MCC

ZNF827

USP3

WDR11

FAM222B

ZNF143

PIK3CB

ING2
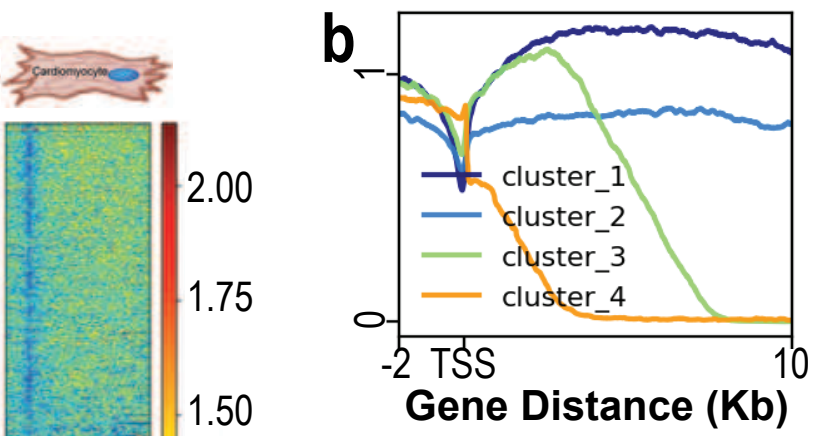

1.25

1.00

0.75
0.50
0.25
0.00

f

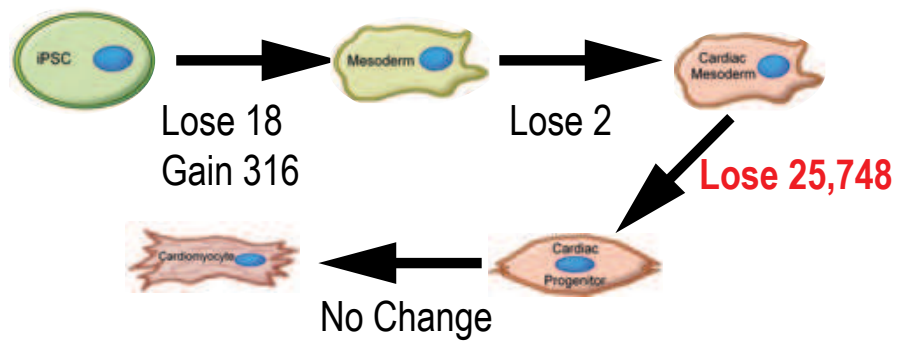

h
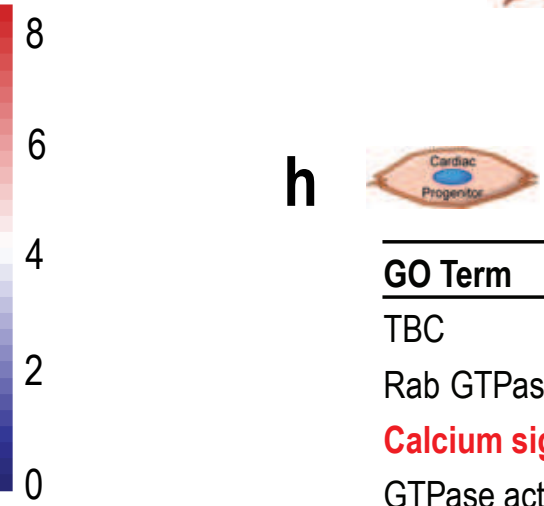

C

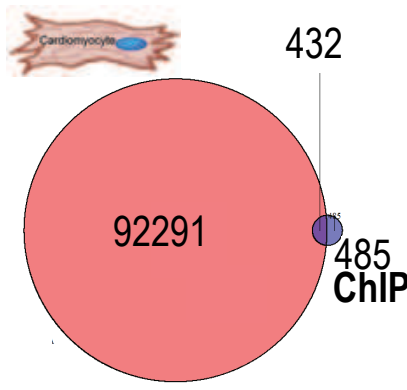

RNA

e

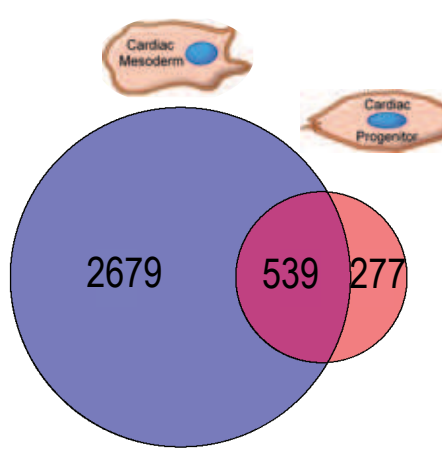

\begin{tabular}{|c|c|c|}
\hline GO Term & Benjamini & \\
\hline TBC & 2.10E -03 & $\begin{array}{l}\text { CACNATE } \\
\text { CACNA1H }\end{array}$ \\
\hline Rab GTPase binding & $7.20 \mathrm{E}-03$ & CHRM3 \\
\hline Calcium signaling pathway & $8.90 \mathrm{E}-03$ & ERBB4 \\
\hline GTPase activation & $1.40 \mathrm{E}-02$ & MYLK \\
\hline Rab-GTPase TBCdomain & $1.50 \mathrm{E}-02$ & PDE1A \\
\hline GTPase activator activity & $3.00 \mathrm{E}-02$ & PDE1C \\
\hline $\begin{array}{l}\text { Cardiomyopath } \\
\text { Non-cardiomyo }\end{array}$ & $\begin{array}{l}\text { les } \\
\text { Genes }\end{array}$ & $\begin{array}{l}\text { PLCB1 } \\
\text { RYR2 } \\
\text { TACR1 }\end{array}$ \\
\hline
\end{tabular}

Calcium Genes

$647 \mathrm{~Kb}$

$\mathbf{i}$

\section{C}

NA1C

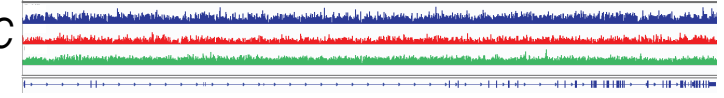

$794 \mathrm{~Kb}$
Non-calcium Genes

$95 \mathrm{~Kb}$

KDM7A

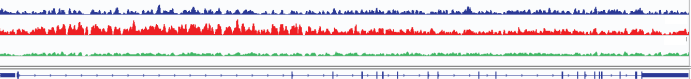

$41 \mathrm{~Kb}$
IPSC

Mesoderm

Cardiomyocyte 
736 Figure 2: H2Bub1 in iPSC-derived cardiomyocyte development shows selective

737 maintenance of sarcomeric calcium genes

738 a) H2Bub1 surrounding the transcriptional start site (TSS) $(-2 \mathrm{~Kb},+10 \mathrm{~Kb})$ in five stages of CM

739 differentiation (iPSC, mesoderm (M), cardiac mesoderm (CMes), cardiac progenitor (CP),

740 and cardiomyocyte (CM)). Genes are grouped by H2Bub1 occupancy into four clusters. The

741 average profile, depicted using fold enrichment against random distribution values, across

742 this region for each cluster in each stage is shown. This represents the average of three

743 replicates.

744 b) The cluster breakdown for (a). Cluster 1 indicates high H2Bub1 levels, cluster 2 indicates low

745 H2Bub1 levels, cluster 3 indicates moderate H2Bub1 levels, and cluster 4 indicates no

$746 \quad$ H2Bub1.

747 c) Comparison of expressed transcripts from RNA-seq data (red) to the Ensembl transcripts near

$748 \quad \mathrm{H} 2 \mathrm{Bub1}$ peaks from ChIP-seq data (blue) at the CM stage.

749 d) Western blot for H2Bub1 in five stages of CM differentiation (iPSC, M, CMes, CP, and

$750 \mathrm{CM}$ ). The loading control for H2Bub1 is H2B. Numbers indicate average quantification

751 (imageJ) of H2Bub1 normalized to H2B over two replicates.

752 e) Comparison of the number of Ensembl genes located near H2Bub1 peaks based on ChIP-seq

753 data from CMes (blue) to CP (red).

754 f) Results of a differential binding analysis for $\mathrm{H} 2 \mathrm{Bub1-ChIP-seq} \mathrm{across} \mathrm{five} \mathrm{stages} \mathrm{of} \mathrm{CM}$

755 differentiation (iPSC, M, CMes, CP, and CM). This depicts the number of regions that gain

756 and lose H2Bub1 at each transition.

757 g) A heat map depicting the top 20 significant regions with increased H2Bub1 occupancy in $\mathrm{M}$

$758 \quad$ compared with iPSCs. 
759 h) The significant gene ontology terms from the genes near regions that maintain H2Bub1

760 between CMes and CP. Genes associated with the calcium signaling pathway are listed and

761 are colored blue if they are associated with cardiomyopathy from patient variants and/or

762 mouse models and are colored black if they are not.

763 i) Example H2Bub1 occupancy, depicted using fold enrichment against random distribution

764 across calcium genes (CACNAIC and RYR2, values ranging from 0 to 3 ) and non-calcium

765 genes (KDM7A and NAT10, values ranging from 0 to 8) at three stages in CM differentiation

766 (iPSC (blue), M (red), and CM (green)). $11 \mathrm{o}$ the genes are oriented 5 to 3 . ene structure

767 is indicated below the gene. 


\section{Figure 3: Reduced total H2Bub1 levels lead to abnormal cardiomyocytes}

769 a) The percent of each 6 well plate of iPSC-derived cardiomyocytes that beats by day 20 is

770 shown with colored dots (WT $(\mathrm{n}=12)$ is blue, $R N F 20^{+/-}($mutant $1: \mathrm{n}=8$, mutant $2: \mathrm{n}=11)$ is

771 red, and $U B E 2 B^{-/-}$(mutant $1: \mathrm{n}=18$, mutant $2: \mathrm{n}=11$ ) is green). Data are shown as

772 individual data points and a blue line representing the median. Unpaired 2-tailed,

773 heteroscedastic t-test, $* \mathrm{p}<0.05, * * \mathrm{p}<0.01$, N.S. is not significant.

774 b) Transmission electron microscopy of e12.25 wild-type $\left(\operatorname{Rnf} 20^{\mathrm{fl} /+}:: N k x 2.5 \mathrm{Cre}^{+}, \mathrm{n}=2\right)$ and

775 mutant $\left(\operatorname{Rnf} 20^{\mathrm{fl} \times /-}:: N k x 2.5 \mathrm{Cre}^{+}, \mathrm{n}=2\right)$ mouse heart sarcomeres. 


\section{Figure 4}

a $\begin{array}{lllll}\text { iPSC } & M & \text { CMT } & \text { CP } & \text { CM }\end{array}$
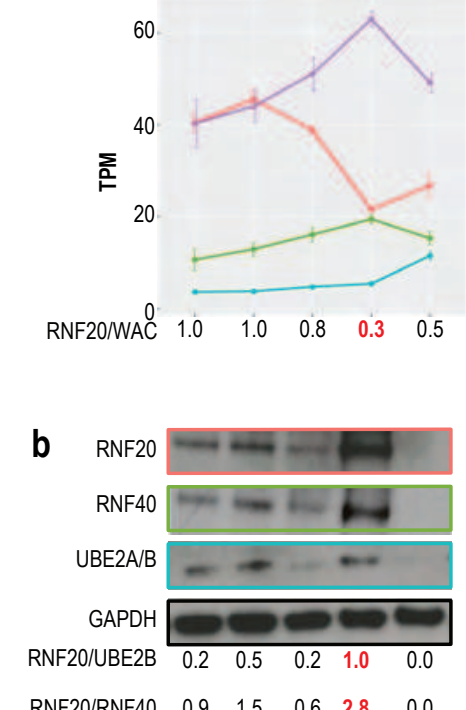

$\begin{array}{llllll}\text { RNF20/RNF40 } & 0.9 & 1.5 & 0.6 & 2.8 & 0.0\end{array}$

C

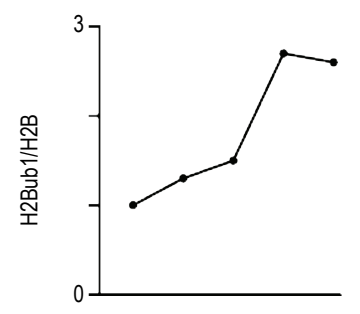

d iPSC

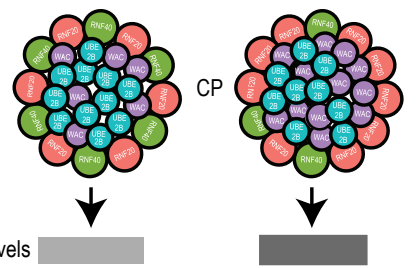

H2Bub1 Levels

Low

UBE2B Knockout

e Stage iPSC IPSC IPSC $M \quad M \quad M$

UBE2B $\quad+/+\quad-/-1 \quad-/-2 \quad+/+\quad-/-1 \quad-/ / 2$

RNF20 L * ? and int?

RNF40

UBE2A/B

GAPDH

RNF20/UBE2B

$\begin{array}{llllll}0.1 & 0.3 & 0.2 & 0.5 & 0.7 & 0.4\end{array}$

RNF20/RNF40

$\begin{array}{llllll}1.0 & 0.6 & 0.5 & 1.3 & 0.8 & 0.4\end{array}$
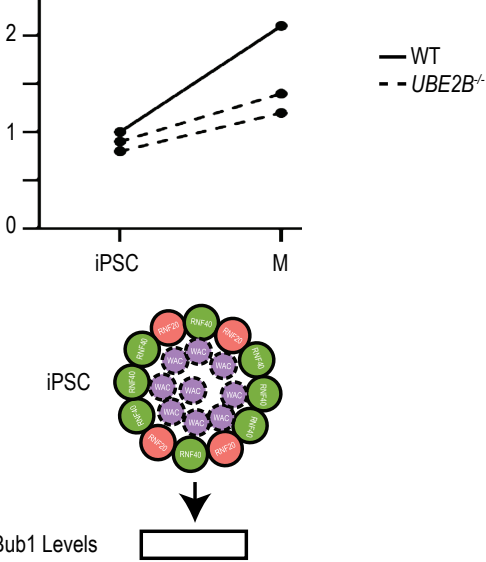

h

RNF20 Haploinsufficient

iPSC M

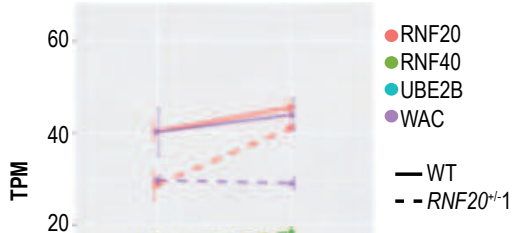

RNF2O/WAC

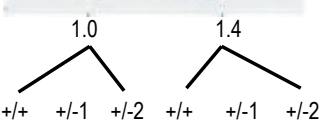

i

RNF20 $\quad+/+\quad+/-1 \quad+/-2 \quad+/+\quad+/ /-1 \quad+/-2$

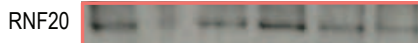

RNF40 I $1+2=4$

UBE2A/B

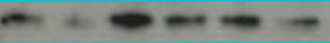

GAPDH

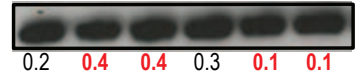

$\begin{array}{lllllll}\text { RNF20/UBE2B } & 0.2 & 0.4 & 0.4 & 0.3 & 0.1 & 0.1\end{array}$

$\begin{array}{lllllll}\text { RNF20/RNF40 } & 1.4 & 0.4 & 0.9 & 1.1 & 0.9 & 0.8\end{array}$

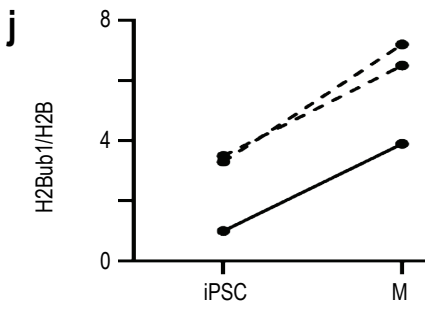

-WT
-- RNF20

k

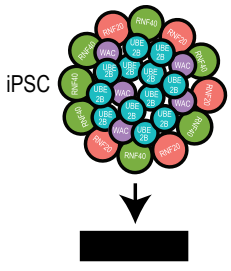

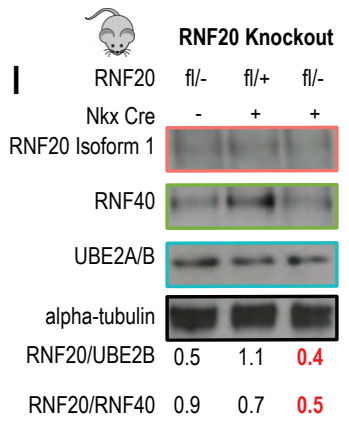

m

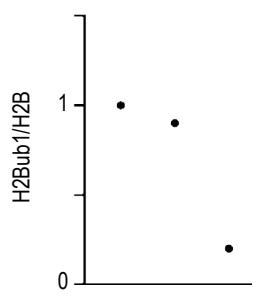

n

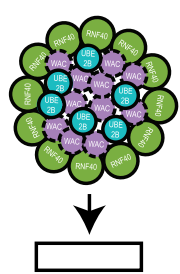




\section{Figure 4: H2Bub1 level is determined by ratios of RNF20-complex components}

777 a) RNA-seq data for RNF20-complex components (RNF20, RNF40, UBE2B, and WAC) in five

778 stages of CM differentiation (iPSC, mesoderm (M), cardiac mesoderm (CMes), cardiac

779 progenitor $(\mathrm{CP})$, and cardiomyocyte $(\mathrm{CM}))$ in wild-type cells. Data are shown as mean \pm

780 SEM $(\mathrm{n}=3)$. Numbers indicate average ratio of $R N F 20$ to WAC mRNA over three replicates.

781 b) Western blot for RNF20-complex components (RNF20, RNF40, and UBE2B) in five stages

782 of CM differentiation in wild-type cells. The loading control is GAPDH. The upper numbers

783 indicate average quantification (imageJ) of the ratio of RNF20 normalized to GAPDH over

784 UBE2B normalized to GAPDH for three replicates. The lower numbers indicate average

785 quantification (imageJ) of the ratio of RNF20 normalized to GAPDH over RNF40

786 normalized to GAPDH for three replicates.

787 c) Quantified western blots (imageJ) for $\mathrm{H} 2 \mathrm{Bub} 1$ normalized to $\mathrm{H} 2 \mathrm{~B}$ in five stages of $\mathrm{CM}$

788 differentiation in wild-type cells. Data are shown as the mean $(n=2)$.

789 d) Schematic depicting predicted ratio of the complex components (based on protein level for

790 RNF20, RNF40 and UBE2B/A and mRNA level for WAC all determined by mean of three

791 replicates) at the stage where $\mathrm{H} 2 \mathrm{Bub} 1$ is the lowest (iPSC) and at the stage where $\mathrm{H} 2 \mathrm{Bub} 1$ is

792 the highest (CP) and the corresponding H2Bub1 levels. RNF20-red, RNF40-green,

$793 \quad$ UBE2A/B blue, WAC-purple.

794 e) Western blot for RNF20-complex components (RNF20, RNF40, and UBE2B) in wild-type

795 and $U B E 2 B^{--}$mutant iPSC and M cells. The loading control is GAPDH. The upper numbers

796 indicate average quantification (imageJ) of the ratio of RNF20 normalized to GAPDH over

797 UBE2B normalized to GAPDH for three replicates. The lower numbers indicate average 
799 normalized to GAPDH for three replicates.

800 f)Quantified western blots (imageJ) for $\mathrm{H} 2 \mathrm{Bub} 1$ normalized to $\mathrm{H} 2 \mathrm{~B}$ in wild-type and $U B E 2 B^{-/-}$

801 mutant iPSC and M cells. Data are shown as the mean $(\mathrm{n}=3)$.

802 g) Schematic depicting predicted ratio of the complex components (based on protein level for

803 RNF20, RNF40 and UBE2B/A all determined by mean of three replicates; WAC is assumed

804 based on WT data) at the iPSC stage in the $U B E 2 B^{-/-}$cells and the corresponding H2Bub1

805 level. RNF20-red, RNF40-green, WAC-purple.

806 h) RNA-seq data for RNF20-complex components (RNF20, RNF40,UBE2B, and WAC) in

807 wild-type and $R N F 20^{+/} 1$ mutant iPSC and M cells. Data are shown as mean \pm SEM $(\mathrm{n}=3)$.

808 Numbers indicate average ratio of $R N F 20$ to WAC mRNA in the $R N F 20^{+/} 1$ mutant cells over 809 three replicates.

810 i) Western blot for RNF20-complex components (RNF20, RNF40, and UBE2B) in wild-type and $811 \mathrm{RNF} 2 \mathrm{O}^{+-}$mutant iPSC and M cells. The loading control is GAPDH. The upper numbers

812 indicate average quantification (imageJ) of the ratio of RNF20 normalized to GAPDH over

813 UBE2B normalized to GAPDH for three replicates. The lower numbers indicate average

814 quantification (imageJ) of the ratio of RNF20 normalized to GAPDH over RNF40

815 normalized to GAPDH for three replicates.

816 j)Quantified western blots (imageJ) for H2Bub1 normalized to H2B in wild-type and $R N F 20^{+/-}$

817 mutant iPSC and M cells. Data are shown as the mean $(\mathrm{n}=3)$.

818 k) Schematic depicting predicted ratio of the complex components (based on protein level for 819 RNF20, RNF40 and UBE2B/A and mRNA level for WAC all determined by mean of three 
820 replicates) at the iPSC stage in the $R N F 2 O^{+/}$cells and the corresponding H2Bub1 level.

821 RNF20-red, RNF40-green, UBE2A/B blue, WAC-purple.

822 l) Western blot for RNF20-complex components (RNF20, RNF40, and UBE2B) in e12.25 wild-

$823 \quad$ type $\left(\operatorname{Rnf} 20^{\mathrm{fl} /-}:: N k x 2.5 \mathrm{Cre}^{-}\right.$and $\left.\operatorname{Rnf} 20^{\mathrm{fl} /+}:: N k x 2.5 \mathrm{Cre}^{+}\right)$and mutant $\left(\operatorname{Rnf} 20^{\mathrm{fl} /-}:: N k x 2.5 \mathrm{Cre}^{+}\right)$

824 mouse hearts. The loading control is alpha-tubulin. The upper numbers indicate

825 quantification (imageJ) of the ratio of RNF20 normalized to alpha-tubulin over UBE2B

826 normalized to alpha-tubulin. The lower numbers indicate quantification (imageJ) of the ratio

827 of RNF20 normalized to alpha-tubulin over RNF40 normalized to alpha-tubulin.

828 m) Quantified western blot (imageJ) for H2Bub1 normalized to H2B in e12.25 wild-type

$829 \quad\left(\operatorname{Rnf} 20^{\mathrm{fl} /-}:: N k x 2.5 \mathrm{Cre}^{-}\right.$and $\left.\operatorname{Rnf} 20^{\mathrm{fl} /+}:: N k x 2.5 \mathrm{Cre}^{+}\right)$and mutant $\left(\operatorname{Rnf} 20^{\mathrm{fl} /-}:: N k x 2.5 \mathrm{Cre}^{+}\right)$mouse

830 hearts.

831 n) Schematic depicting predicted ratio of the complex components (based on protein level for

832 RNF20, RNF40 and UBE2B/A; WAC is assumed based on WT data) at e12.25 in Rnf $20^{\mathrm{fl} /-}$

833 ::Nkx2.5Cre ${ }^{+}$and the corresponding H2Bub1 level. RNF40-green, UBE2A/B blue, WAC-

834 purple. 
Figure 5

a

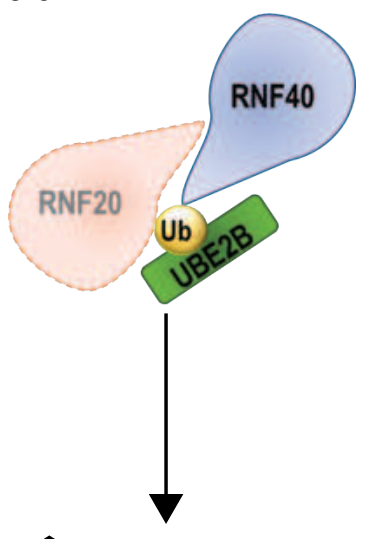

个Total H2Bub1

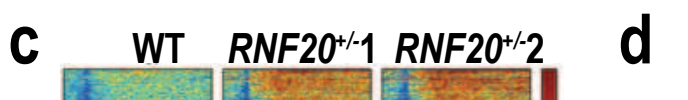

๖ั๊

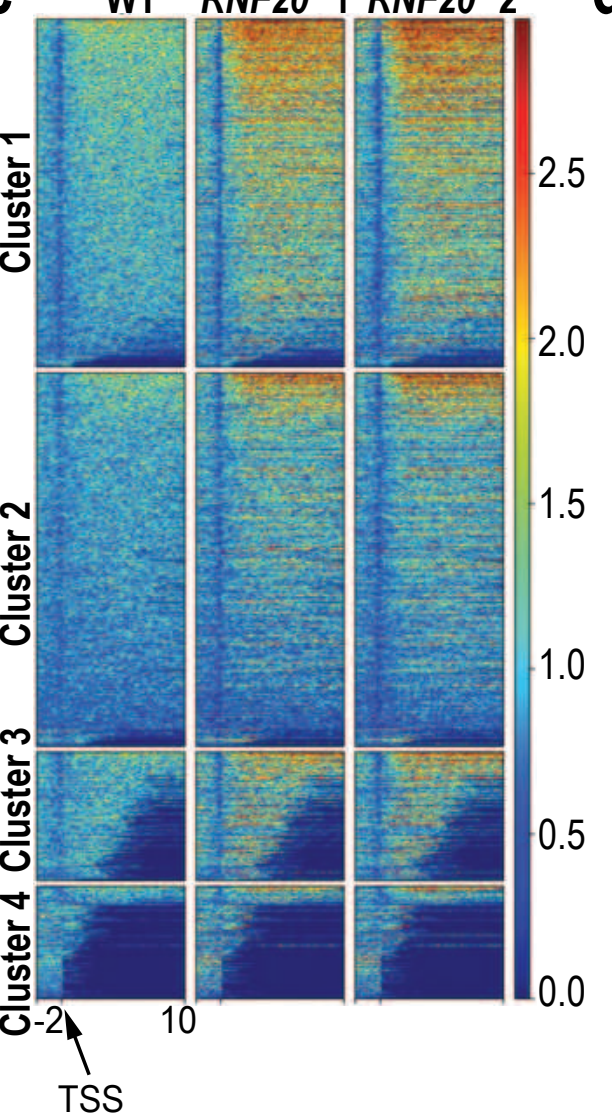

b WT v. RNF20+/iPSC
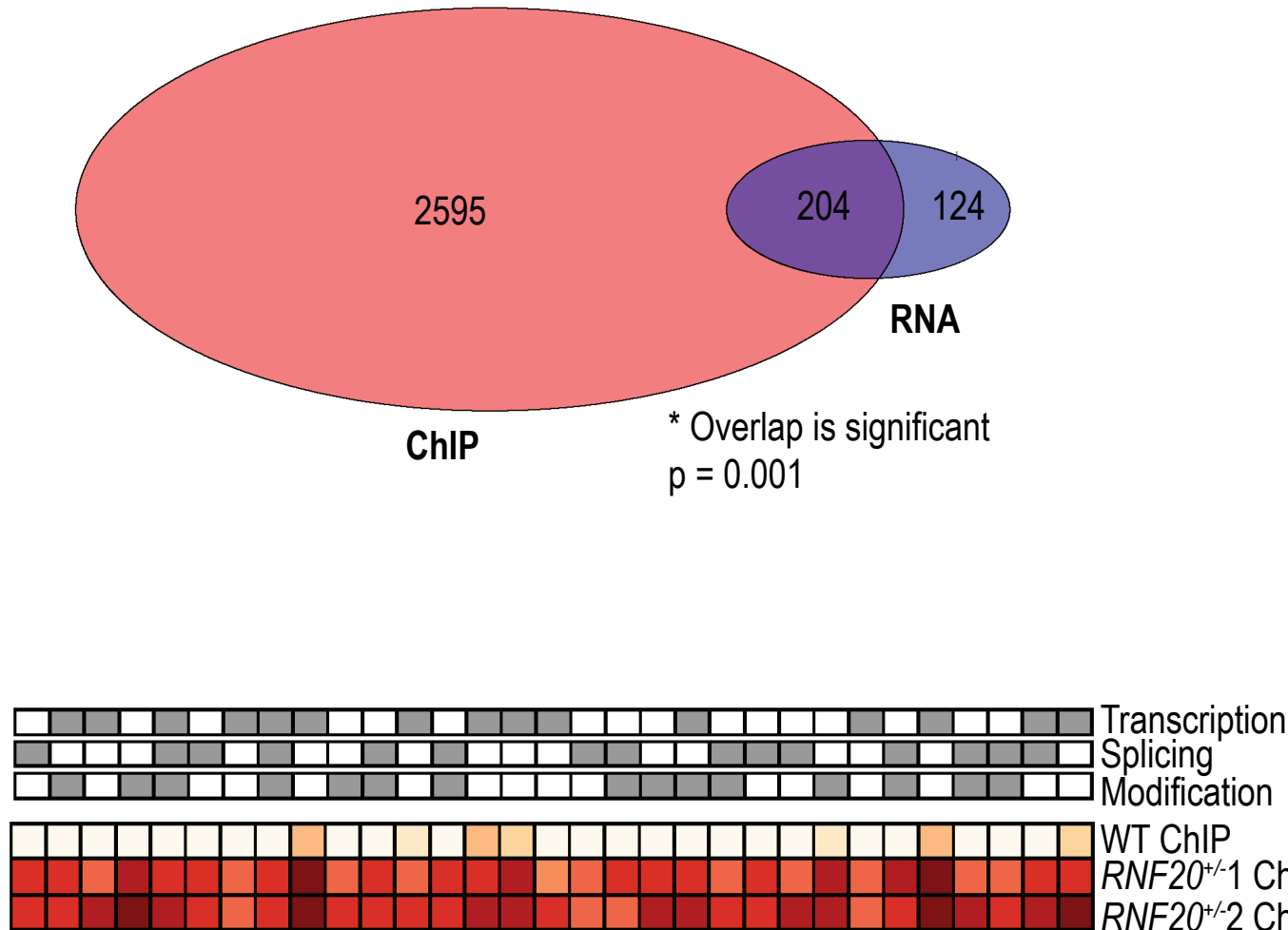

Modification

WT ChIP

$R N F 20^{+-1}$ ChIP RNF20+-2 ChIP

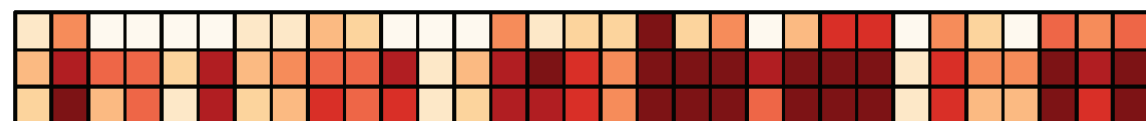

WT RNA

$R N F 20^{+-1}$ RNA $R N F 20^{+}-2$ RNA

Х๐NO

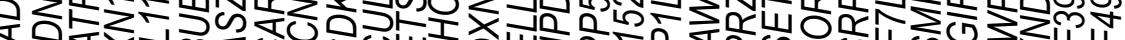

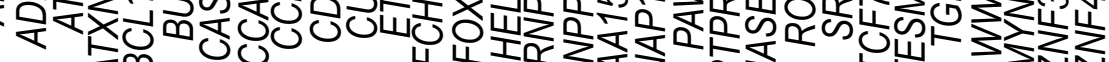

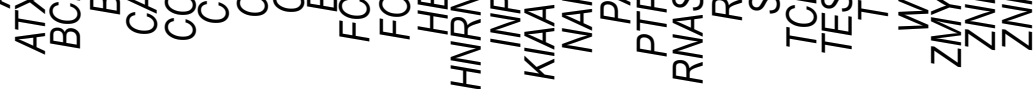

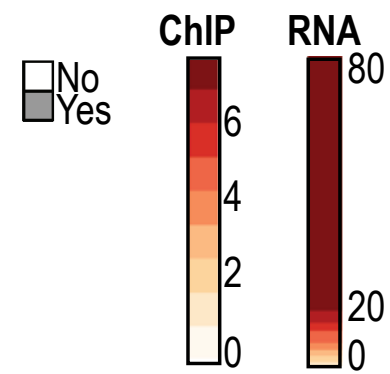




\section{Figure 5: Haploinsufficiency for Rnf20 increases total H2Bub1 in iPSCs and arrests}

\section{6 differentiation into cardiac mesoderm}

837 a) RNF20-complex schematic illustrating the $R N F 20^{+/}$iPSC mutant, and the associated 838 increased total H2Bub1 levels.

839 b) Genes with differing H2Bub1 occupancy (red) and gene expression (blue) when compared to 840 wild-type that are shared between the two independent $R N F 20^{+/}$cell lines at the iPSC stage. 841 signi icant num er o these genes overlap ( isher s exact test $n \quad 3$ ).

842 c) H2Bub1 surrounding the transcriptional start site (TSS) $(-2 \mathrm{~Kb},+10 \mathrm{~Kb})$ in the wild-type and $843 R N F 20^{+-}$mutants at the iPSC stage. Genes are grouped by H2Bub1 levels into four clusters.

844 The average profile, depicted using fold enrichment against random distribution values,

845 across this region for each cluster in each stage is shown. This represents the average of three 846 replicates.

847 d) Gene ontology analysis of genes with differing H2Bub1 occupancy between wild-type and 848 both independent $R N F 2 O^{+/}$cell lines at the iPSC stage. Many of these genes are related to 849 transcription, splicing, and/or DNA/Protein modifications. Expression and H2Bub1

850 occupancy levels for example genes in each category are shown. Gene ontology (upper 851 panel) is in gray scale; ChIP data (middle panel) and mRNA expression data (lower panel) 852 are shown as heat maps. 
853 Figure 6: Sarcomeric calcium signaling gene expression is reduced in cells with decreased 854 total H2Bub1 levels

855 a) RNF20-complex schematic illustrating the $U B E 2 B^{-/-}$iPSC mutant and the $R n f 20^{f /-}$ mouse 856 mutant. Both of these mutations lead to decreased total H2Bub1 levels. The mouse or cell 857 icons in (b) and (c) indicate whether the analysis was done on mouse or cells.

858 b) Quantitative RT-PCR of calcium signaling genes in e11.5 wild-type $\left(\operatorname{Rnf} 20^{\mathrm{fl} /+}:: N k x 2.5 \mathrm{Cre}^{+}\right)$ 859 and mutant $\left(\operatorname{Rnf} 20^{\mathrm{ft} /-}:: N k x 2.5 \mathrm{Cre}^{+}\right)$mouse hearts for Cacnalc, Ryr2, Ncx, and Serca2a. Levels 860 of expression are normalized to $18 s$ rRNA. Individual data points are shown in black dots.

861 Data are shown as mean $\pm \operatorname{SEM}(\mathrm{n}=2)$. Unpaired 1-tailed, heteroscedastic $\mathrm{t}$-test, $* \mathrm{p}<0.05$, $862 * * \mathrm{P}<0.01$, N.S. is non-significant.

863 c) Gene ontology analysis on genes with differing gene expression levels comparing wild-type 864 to both independent $U B E 2 B^{-/}$cell lines at the cardiomyocyte stage. Many of these genes are 865 related to sarcomere, cardiomyopathy, and/or calcium signaling. Expression levels for 866 example genes in each category are shown. Stars indicate genes shared with maintained 867 H2Bub1 marks upon transition to CPs shown in Fig. 2h. Data represent three RNA-seq 868 replicates. Gene ontology (upper panel) is in gray scale; mRNA expression data (lower 869 panel) is shown as a heat map. 
870 Figure 7: Accumulation of H2Bub1 near the center of tissue specific genes is correlated

871 with enhanced efficiency of transcriptional elongation

872 a) Metagene plot for $\mathrm{H} 2 \mathrm{Bub} 1$ levels in wild-type (blue) and $U B E 2 B^{-/}$mutants (green and red)

873 across the calcium signaling genes that are differentially expressed between wild-type and

$874 U B E 2 B^{-/}$mutants and/or selectively maintained between cardiac mesoderm and cardiac

875 progenitor stages $(n=3)$. The $*$ indicates the accumulation near the center of the gene.

876 b) Metagene plot for H2Bub1 levels in wild-type (blue) and $U B E 2 B^{-/}$mutants (green and red)

877 across the sarcomeric genes that are differentially expressed between wild-type and $U B E 2 B^{-/}$

878 mutants $(\mathrm{n}=3)$. The $*$ indicates the accumulation near the center of the gene.

879 c) Example metagene plot for $\mathrm{H} 2 \mathrm{Bub} 1$ levels in wild-type (blue) and $U B E 2 B^{-/}$mutants (green

880 and red) across "randomly" selected quantity and size-matched genes to the calcium gene set

$881(\mathrm{n}=3) .30$ "random" metagene plots were created from the calcium gene set and 30

882 "random" metagene plots were created from the sarcomere gene set: 10 from genes that are

883 upregulated between wild-type and both $U B E 2 B^{-/-}$cell lines, 10 that are non-regulated

884 between wild-type and both $U B E 2 B^{-/}$cell lines, and 10 that are down-regulated between

885 wild-type and both $U B E 2 B^{-/}$cell lines.

886 d) $\log 2$ of fold change in transcript abundance between wild-type and $U B E 2 B^{-/}$mutants is

887 shown at each position along the genes. The genes being shown are the calcium signaling

888 genes describe in (a).

889 e) $\log 2$ of fold change in transcript abundance between wild-type and $U B E 2 B^{-/}$mutants is

890 shown at each position along the genes. The genes being shown are the sarcomeric genes

891 described in (b). 
892 f) $\log 2$ of fold change in transcript abundance between wild-type and $U B E 2 B^{-/}$mutants is 893 shown at each position along the genes. The genes being shown are "randomly" selected 894 quantity and size-matched genes to the calcium signaling genes described in (c).

895 g) Example $\log 2$ of fold change gene traces for calcium genes (CACNA1C and RYR2) that have 896 accumulation of $\mathrm{H} 2 \mathrm{Bub} 1$ near the center of the gene and decreased transcriptional elongation 897 efficiency. 5 on the le to the diagram.

898 h) Metagene plot for H2Bub1 levels in wild-type MEFs (teal) and wild-type mESCs (red)

899 across the ECM genes that are differentially expressed between wild-type MEFs and wild-

900 type mESCs. The * indicates the accumulation near the center of the gene.

901 i) Metagene plot for H2Bub1 levels in wild-type MEFs (teal) and wild-type mESCs (red)

902 across the adhesion genes that are differentially expressed between wild-type MEFs and 903 wild-type mESCs. The * indicates the accumulation near the center of the gene.

904 j) Example metagene plot for H2Bub1 levels in wild-type MEFs (teal) and wild-type mESCs 905 (red) across "randomly" selected quantity and size-matched genes to the adhesion gene set.

90620 "random" metagene plots were created from the adhesion gene set and 20 "random"

907 metagene plots were created from the ECM gene set. 
Figure 8

a
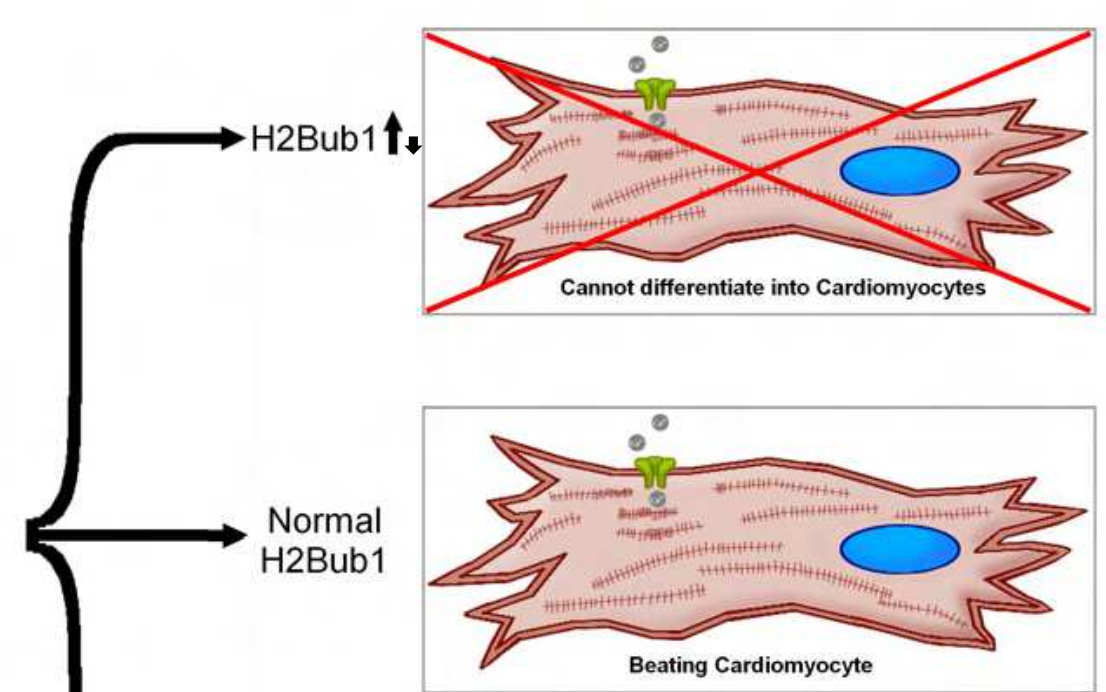

Normal H2Bub1

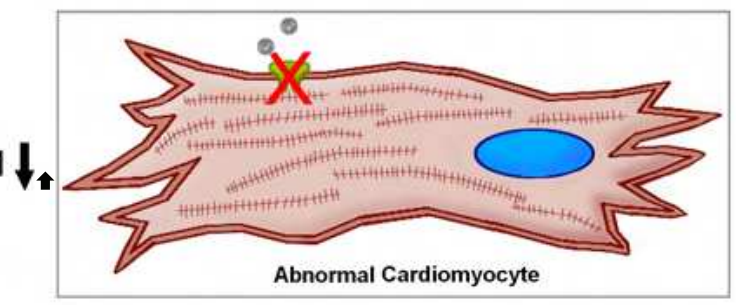

b

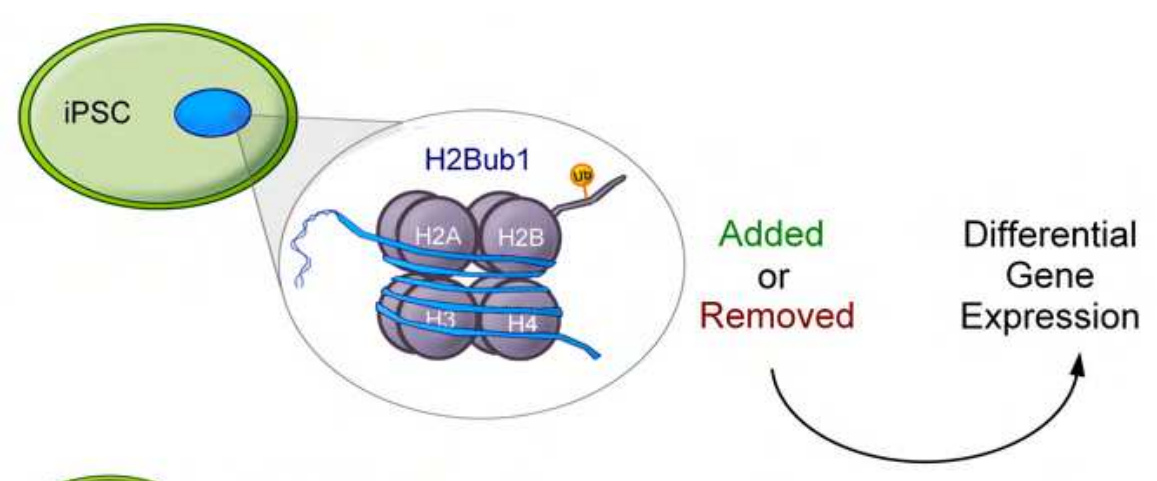

Cardiac
Mesoderm

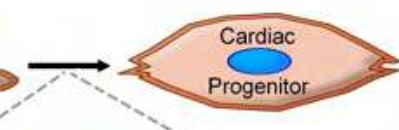

C

Short Genes

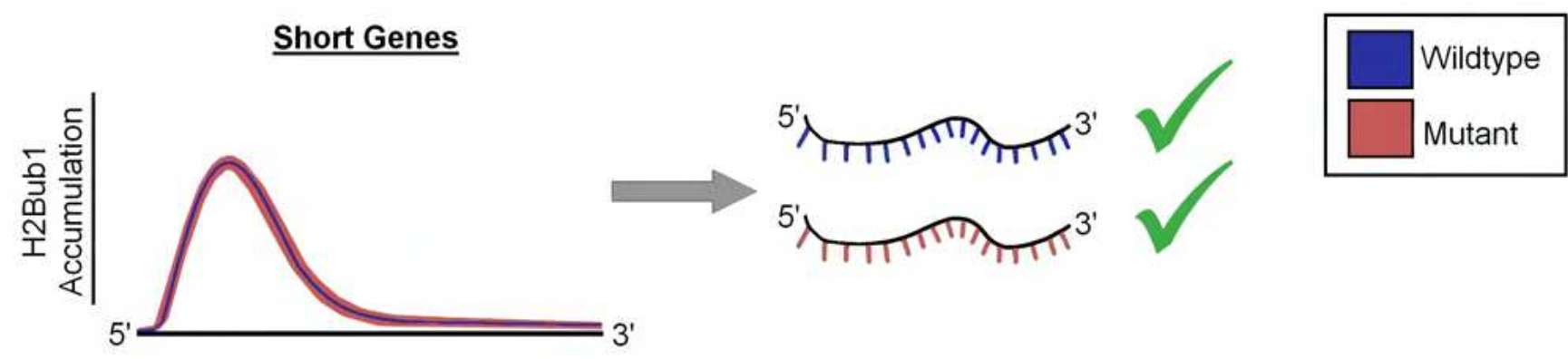

Long Genes

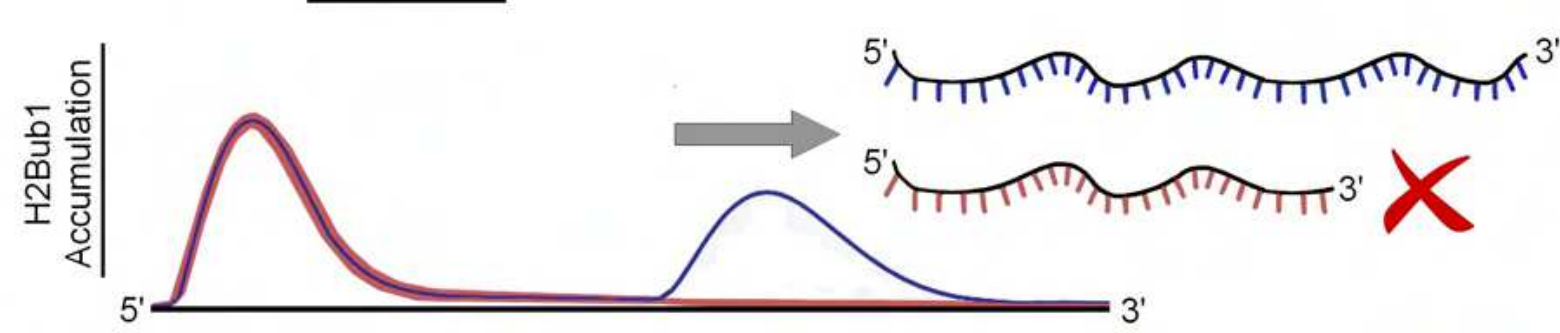




\section{Figure 8: Working Model}

909 a) When H2Bub1 levels are misregulated by altering levels of complex components,

910 cardiomyocytes do not form normally. When total H2Bub1 levels are increased by

911 haploinsufficiency of RNF20, cardiomyocytes do not form. When total H2Bub1 levels are

912 decreased by creating $U B E 2 B^{-/-}$or $R n f 20^{f l /-}$, sarcomeres do not form normally and calcium

$913 \quad$ signaling genes have reduced expression.

914 b) During wild-type CM differentiation, housekeeping genes and heterochromatic regions have

915 dynamic H2Bub1 levels. H2Bub1 is sparsely maintained during the transition from cardiac

916 mesoderm to cardiac progenitor. Notably, selectively maintained genes are enriched for

917 sarcomeric calcium genes.

918 c) enes that are short in length have an increase in $2 \mathrm{u} 1$ at the 5 end o the gene that

919 decreases to ards the 3 end. o ever longer tissue-specific genes have an accumulation in

$920 \mathrm{H} 2 \mathrm{Bub} 1$ near the center of the gene in wild-type cells and not in $U B E 2 B^{-/}$. In the mutants

921 when this accumulation is absent, the transcriptional efficiency is reduced. 

a
e9.5
b

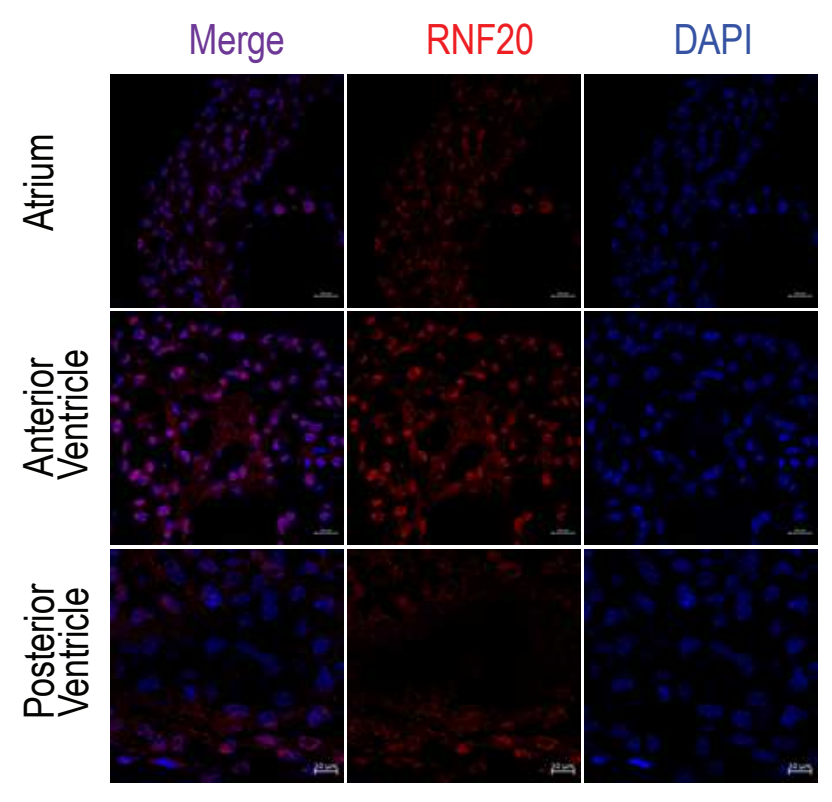

C
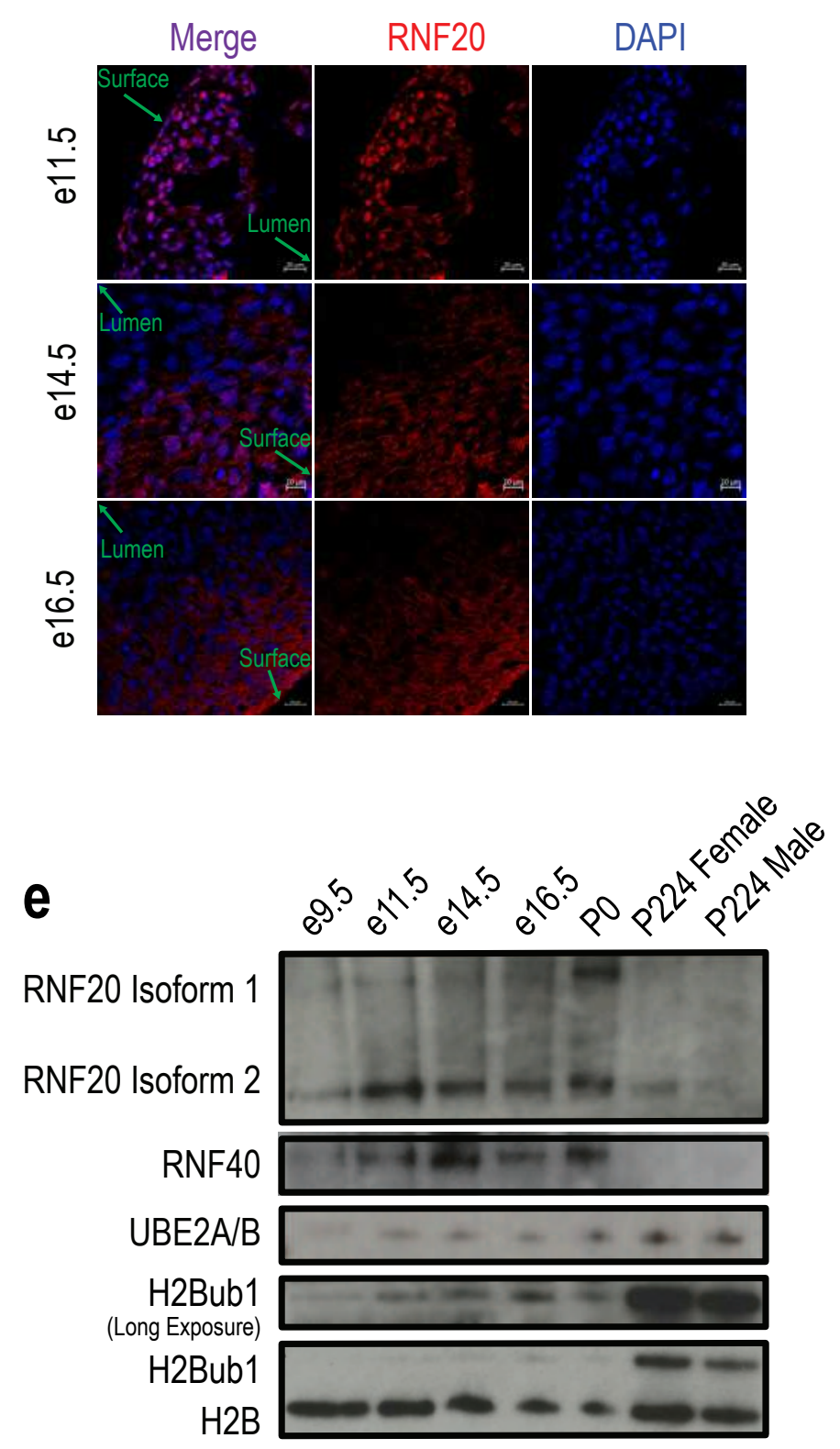
Epicardium
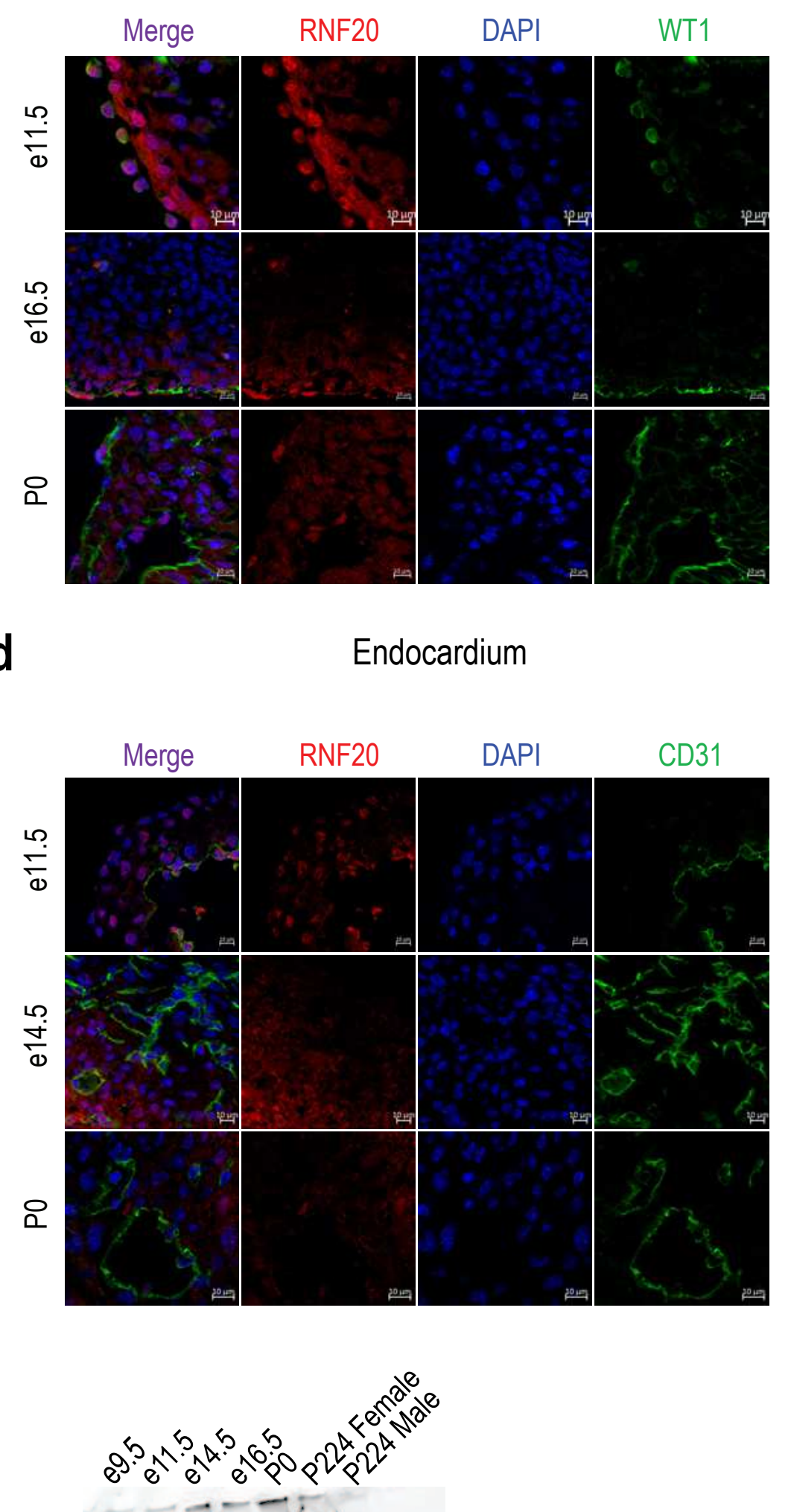


\section{Supplementary Figure 1: RNF20 is dynamically expressed during mouse heart}

\section{3 development}

924 a) Immunofluorescent staining for RNF20 in different sections (atrium, anterior ventricle, and 925 posterior ventricle) of the e9.5 wild-type mouse heart.

926 b) Immunofluorescent staining for RNF20 and WT1 (epicardial marker) in e11.5, e16.5, and P0

927 wild-type mouse hearts. These stages capture the major changes in RNF20 expression in the 928 epicardial cells.

929 c) Immunofluorescence staining for RNF20 in the myocardial cells in e11.5, e14.5, and e16.5

930 wild-type mouse hearts. These stages capture the major changes in RNF20 expression in the 931 myocardial cells.

932 d) Immunofluorescence staining for RNF20 and CD31 (endocardial marker) in e11.5, e14.5, and 933 P0 wild-type mouse hearts. These stages capture the major changes in RNF20 expression in 934 the endocardial cells.

935 e) Western blot for RNF20-complex components (RNF20, RNF40, and UBE2B) and H2Bub1 in 936 wild-type mouse hearts (e9.5, e11.5, e14.5, e16.5, P0, P224 female, and P224 male). The 937 loading control for the complex components is total protein (shown to the right of the 938 western blots) and for $\mathrm{H} 2 \mathrm{Bub} 1$ is $\mathrm{H} 2 \mathrm{~B}$. 


\section{Supplementary Figure 2}

a Rnf20 Wildype $\longrightarrow-\cdots+\cdots$ Allele

Knockout Construct

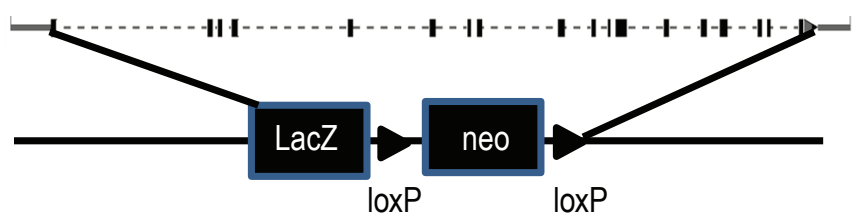

b

\begin{tabular}{ll}
\hline & Rnf20 \\
\hline & P0 \\
\hline$+/+$ & $33.1 \%(346 / 1045)$ \\
$+/-$ & $66.9 \%(699 / 1045)$ \\
-- & $0 \% \quad(0 / 1045)$ \\
\hline
\end{tabular}

Rnf20 Allele (Cre-deleted)

\section{LacZ}
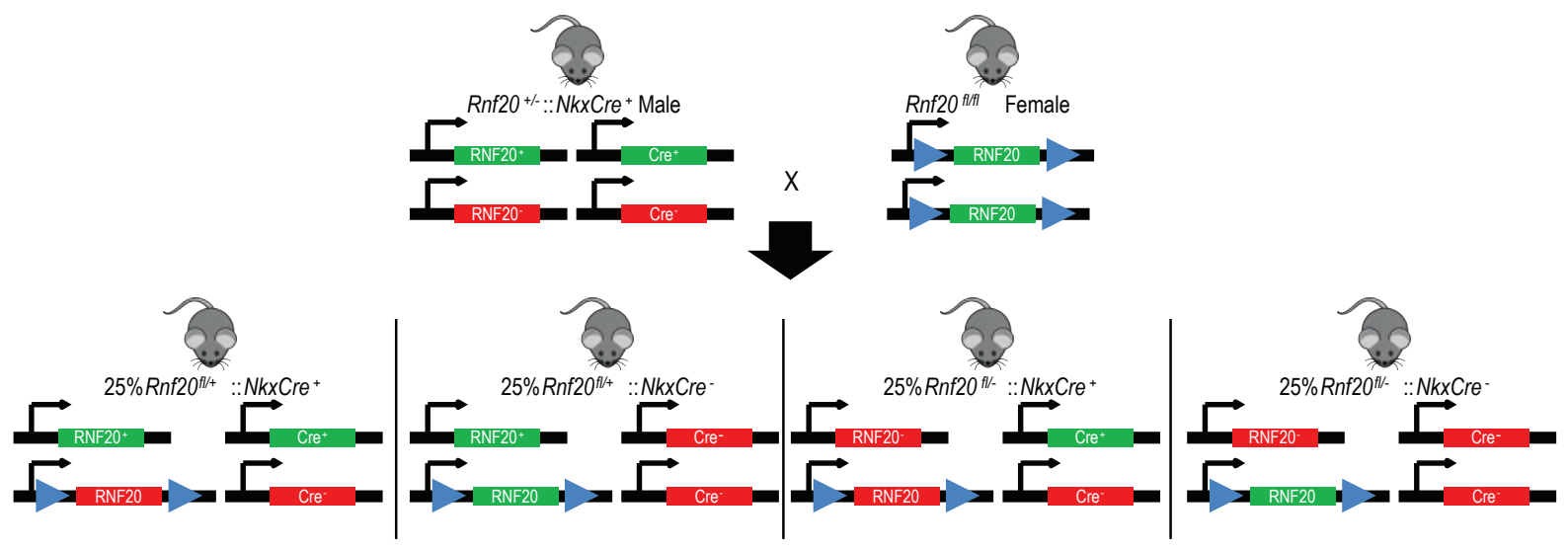

d

\begin{tabular}{|c|c|c|c|c|}
\hline \multicolumn{5}{|c|}{ 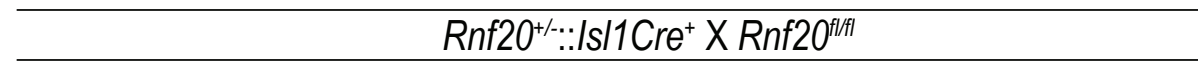 } \\
\hline & e9.5 & e12.5 & P0 & P224 \\
\hline$\overline{f l /+ \text { cret }}$ & $11.1 \%(1 / 9)$ & $34.6 \%(9 / 26)$ & $23.6 \%(13 / 55)$ & $21.4 \%(3 / 14)$ \\
\hline $\mathrm{fl} /+\mathrm{cre}-$ & $44.4 \%(4 / 9)$ & $30.8 \%(8 / 26)$ & $38.2 \%(21 / 55)$ & $21.4 \%(3 / 14)$ \\
\hline $\mathrm{fl} / \mathrm{-cre+}$ & $11.1 \%(1 / 9)$ & $11.5 \%(3 / 26)$ & $23.6 \%(13 / 55)$ & $21.4 \%(3 / 14)$ \\
\hline fl/- cre- & $33.3 \%(3 / 9)$ & $23.1 \%(6 / 26)$ & $14.5 \%(8 / 55)$ & $35.7 \%(5 / 14)$ \\
\hline
\end{tabular}

e

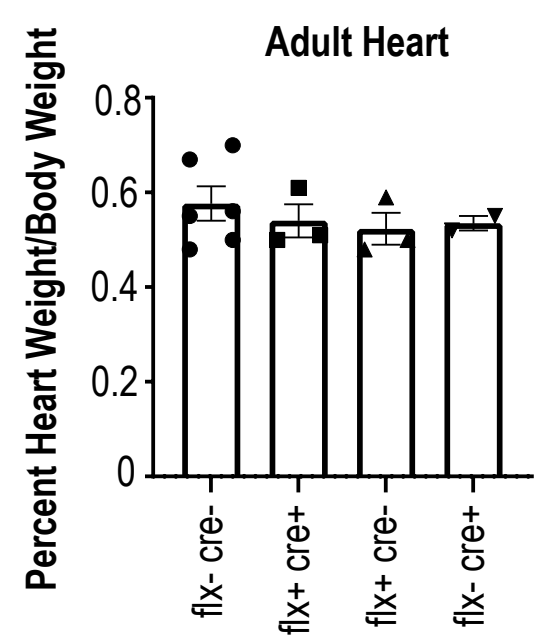

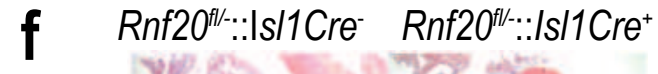

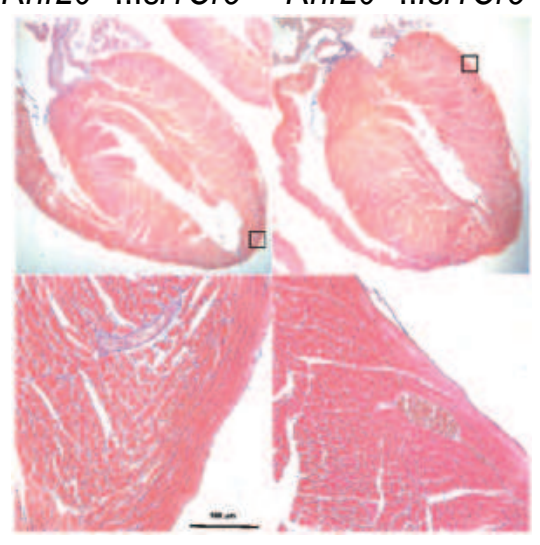

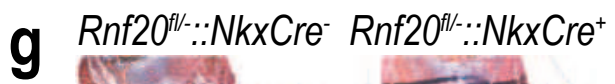

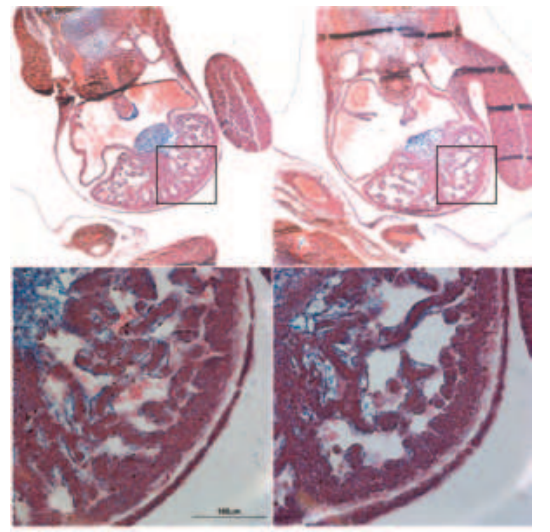

$\mathrm{h}$

\begin{tabular}{llllll}
\hline \multicolumn{5}{c}{ Rnf20 $^{+\ldots . .}:$ Nkx2.5Cre ${ }^{+}$X Rnf20 } \\
\hline & $\mathrm{e} 9.5$ & $\mathrm{e} 11.5$ & $\mathrm{e} 12.25$ & $\mathrm{e} 12.5$ & $\mathrm{P} 0$ \\
\hline $\mathrm{fl} /+\mathrm{cre}+$ & $35 \%(7 / 20)$ & $25.6 \%(46 / 180)$ & $20.5 \%(34 / 166)$ & $55 \%(11 / 20)$ & $35 \%(21 / 60)$ \\
$\mathrm{fl} /+$ cre- & $10 \%(2 / 20)$ & $27.8 \%(50 / 180)$ & $35.5 \%(59 / 166)$ & $25 \%(5 / 20)$ & $43.3 \%(26 / 60)$ \\
$\mathrm{fl} /$ - cre+ & $25 \%(5 / 20)$ & $26.7 \%(48 / 180)$ & $20.5 \%(34 / 166)$ & $5 \%(1 / 20)$ & $0 \%(0 / 60)$ \\
$\mathrm{fl} /$ - cre- & $30 \%(6 / 20)$ & $20 \%(36 / 180)$ & $23.5 \%(39 / 166)$ & $15 \%(3 / 20)$ & $21.7 \%(13 / 60)$ \\
\hline
\end{tabular}




\section{Supplementary Figure 2: Breeding strategy and phenotypes of Rnf20 mutants}

940 a) Diagram illustrating the Rnf20 deletion construct. This mouse was created by knocking out

941 exons 2-19 (exon 1 is non-coding and exon 20 is mostly non-coding) and replacing them

942 with a LacZ neo cassette. The neo cassette was dropped using the LoxP sites.

943 b) Percent of each genotype of P0 mice born from the cross of two $R n f 20^{+/}$mice.

944 c) Diagram illustrating the mouse breeding scheme for the conditional knock-out mice.

945 d) Percent of each genotype of e9.5, e12.5, P0, and P224 from the cross of Rnf $20^{f l / f 1}$ and $R n f 20^{+/-}$

$946 \quad:: I s l l \mathrm{Cre}^{+}$.

947 e) Heart weight/body weight ratios for $14\left(6\right.$ Rnf $20^{f / /}:: I s l l \mathrm{Cre}^{-}, 3 \mathrm{Rnf} 20^{f l /+}:: I s l l \mathrm{Cre}^{+}, 3$

$948 R \operatorname{Rnf} 20^{f l /+}:: I s l l \mathrm{Cre}^{-}$, and $2 \operatorname{Rnf} 20^{f l /-}:: I s l l \mathrm{Cre}^{+}$) adult offspring (P224) from the cross of Rnf $20^{f l / f l}$

949 and $\operatorname{Rnf} 20^{+/}:: I s l 1 \mathrm{Cre}^{+}$. Data are shown as mean \pm SEM.

950 f) Example hematoxylin and eosin stained $\mathrm{P} 224$ wild-type $\left(\operatorname{Rnf} 20^{\mathrm{fl} /-}:: I s l l \mathrm{Cre}^{-}\right)$and mutant

$951 \quad\left(\right.$ Rnf $\left.20^{\mathrm{fl} /}: \because I s l l \mathrm{Cre}^{+}\right)$mouse hearts.

952 g) Example hematoxylin and eosin stained e11.5 wild-type (Rnf2 $\left.20^{\mathrm{fl} /}: \because N k x 2.5 \mathrm{Cre}-\right)$ and mutant

$953\left(\operatorname{Rnf} 20^{\mathrm{fl} /-}:: N k x 2.5 \mathrm{Cre}^{+}\right)$. Lower panels are higher magnification views of the ventricles.

954 h) Percent of each genotype of e9.5, e11.5, e12.25, e12.5, and P0 from the cross of Rnf20 $0^{f l f l}$ and 955 $\operatorname{Rnf} 20^{+/-}:: N k x 2.5 \mathrm{Cre}^{+}$. 


\section{Supplementary Figure 3}

a

iPSC and Mesoderm

$1 \mathrm{e}+05$

$5 e+04$

ণิ 0

$-5 e+04$

$-1 e+05$

0

$\bullet$

-Mesoderm @iPSC
Mesoderm and CMes

- ${ }^{25000}$

$-25000$

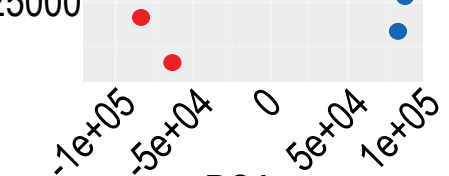

PC1

-CMes

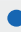

CMes and CP

$2 e+05$

$\mathrm{CP}$ and $\mathrm{MC}$

40000

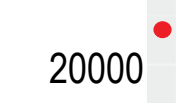

ญ్ฉ

$-20000$
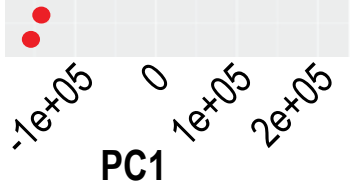

PC1
$1 e+05$

ঠัณ 0

$\bullet$

$-1 e+05$

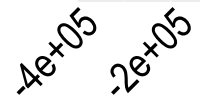

- $\mathrm{CM}$

.

oCMes
C nervous system development cardiovascular system development circulatory system development neuron projection morphogenesis

cell development neuron development neuron projection development cell morphogenesis involved in neuron differentiation vasculature development blood vessel development cellular component assembly involved in morphogenesis neuron differentiation axon development blood vessel morphogenesis cell morphogenesis involved in differentiation heart development regulation of cell differentiation dendrite development regulation of anatomical structure morphogenesis regulation of multicellular organismal development positive regulation of cell differentiation muscle tissue development positive regulation of developmental process central nervous system development alpha-beta T cell differentiation forebrain development

T-helper cell differentiation

CD4-positive, alpha-beta T cell differentiation involved in immune response regulation of nervous system development

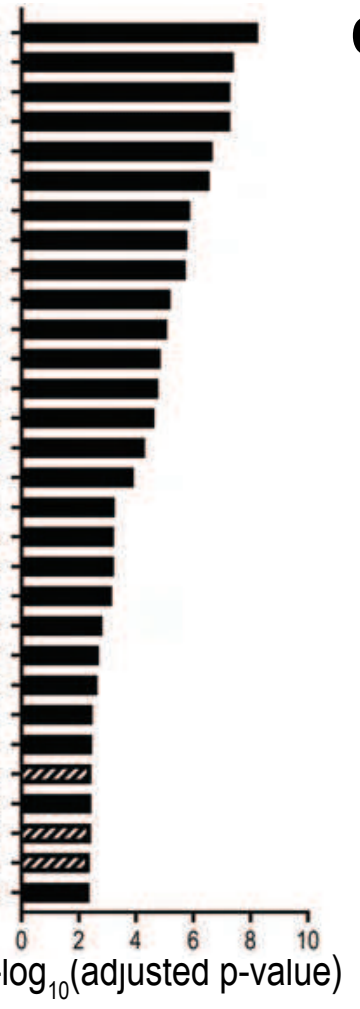

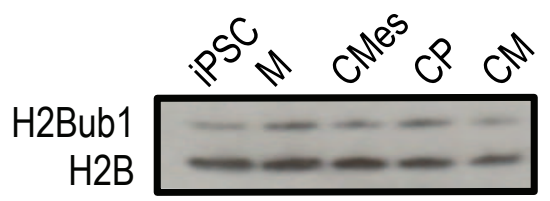

e

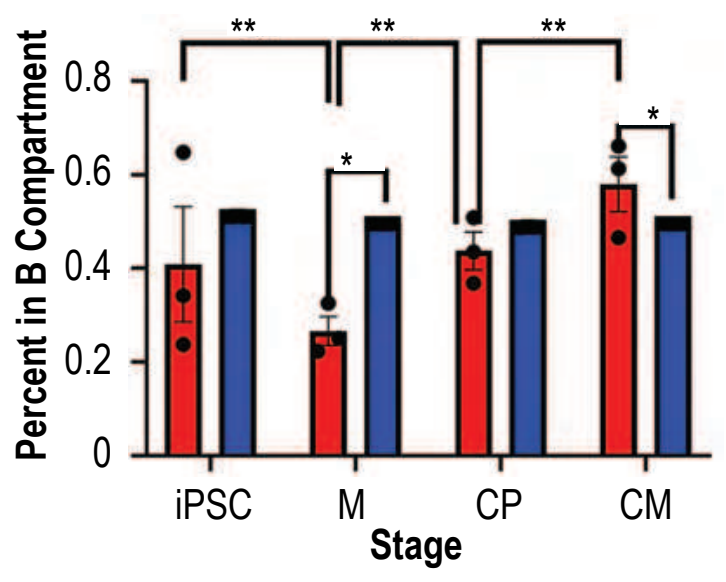

Observed

Expected

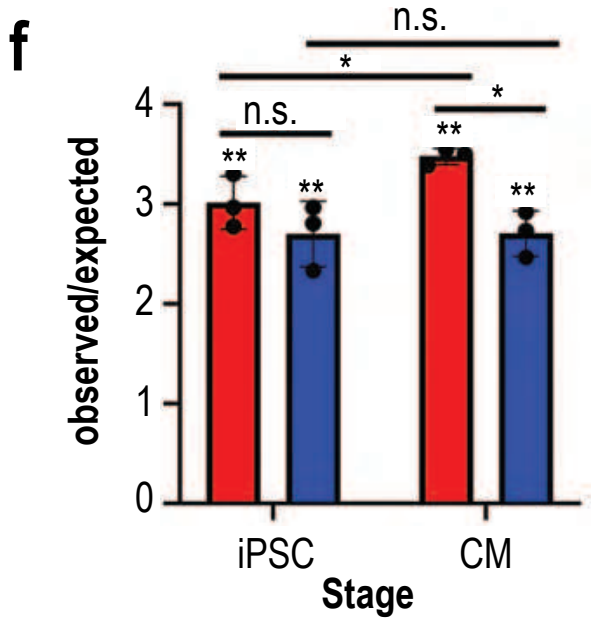

$\mathrm{H} 3 \mathrm{~K} 27 \mathrm{me} 3$

H3K4me3 


\section{Supplementary Figure 3: H2Bub1 and mRNA expression throughout CM differentiation}

957 a) PCA plots of RNA-seq experiments of CM differentiation comparing all neighboring stages

958 (iPSC (red) v mesoderm (M) (blue), M (red) v cardiac mesoderm (CMes) (blue), CMes (red)

959 v cardiac progenitor (CP) (blue), and CP (red) v cardiomyocyte (CM) (blue)) for three

960 replicates of each stage.

961 b) The top 30 gene ontology (GO) terms related to development in cluster 2 (see Fig. 2a, 2b) of

962 iPSCs. Dashed bars represent GO terms not related to heart, nervous system, or appendages.

963 c) The top $30 \mathrm{GO}$ terms related to development in cluster 2 (see Fig. 2a, 2b) of CMs.

964 d) One additional replicate of western blots (see Figure 2d) showing H2Bub1 changes during

965 five stages of CM differentiation (iPSC, M, CMes, CP, and CM). The loading control is

966 H2B.

967 e) Percent of $\mathrm{H} 2 \mathrm{Bub} 1$ peaks overlapping with the $\mathrm{B}$ compartment in each stage of CM

968 differentiation (iPSC, M, CP, and CM) comparing observed (red) and expected (blue) values.

969 Data are shown as mean \pm SEM $(n=3)$. P-values comparing observed and expected values

970 were manually calculated by comparing the actual values to the expected values, $* \mathrm{p}<0.05$,

$971 * * \mathrm{P}<0.01$ (online methods). P-values comparing observed values between stages were

$972 \quad$ calculated by computing a $\mathrm{z}$ score, $* \mathrm{p}<0.05, * * \mathrm{P}<0.01$.

973 f) Percent of $\mathrm{H} 2 \mathrm{Bub} 1$ peaks on the same gene as an activating chromatin mark (H3K4me3

974 shown in blue) and a repressive chromatin mark (H3K27me3 shown in red) in iPSCs and

975 CMs. Data are the ratio of observed to expected values. Data are shown as mean \pm SEM $(n=$

976 3). P-values comparing observed values to expected values were calculated by Chi Square

977 tests and adjusted for multiple comparisons, ${ }^{*} \mathrm{p}<0.05, * * \mathrm{P}<0.01$. P-values comparing 
978 observed values between stages were calculated using an unpaired 2-tailed, heteroscedastic t979 test, $* \mathrm{p}<0.05, * * \mathrm{P}<0.01$, N.S. is non-significant. 
Supplementary Figure 4
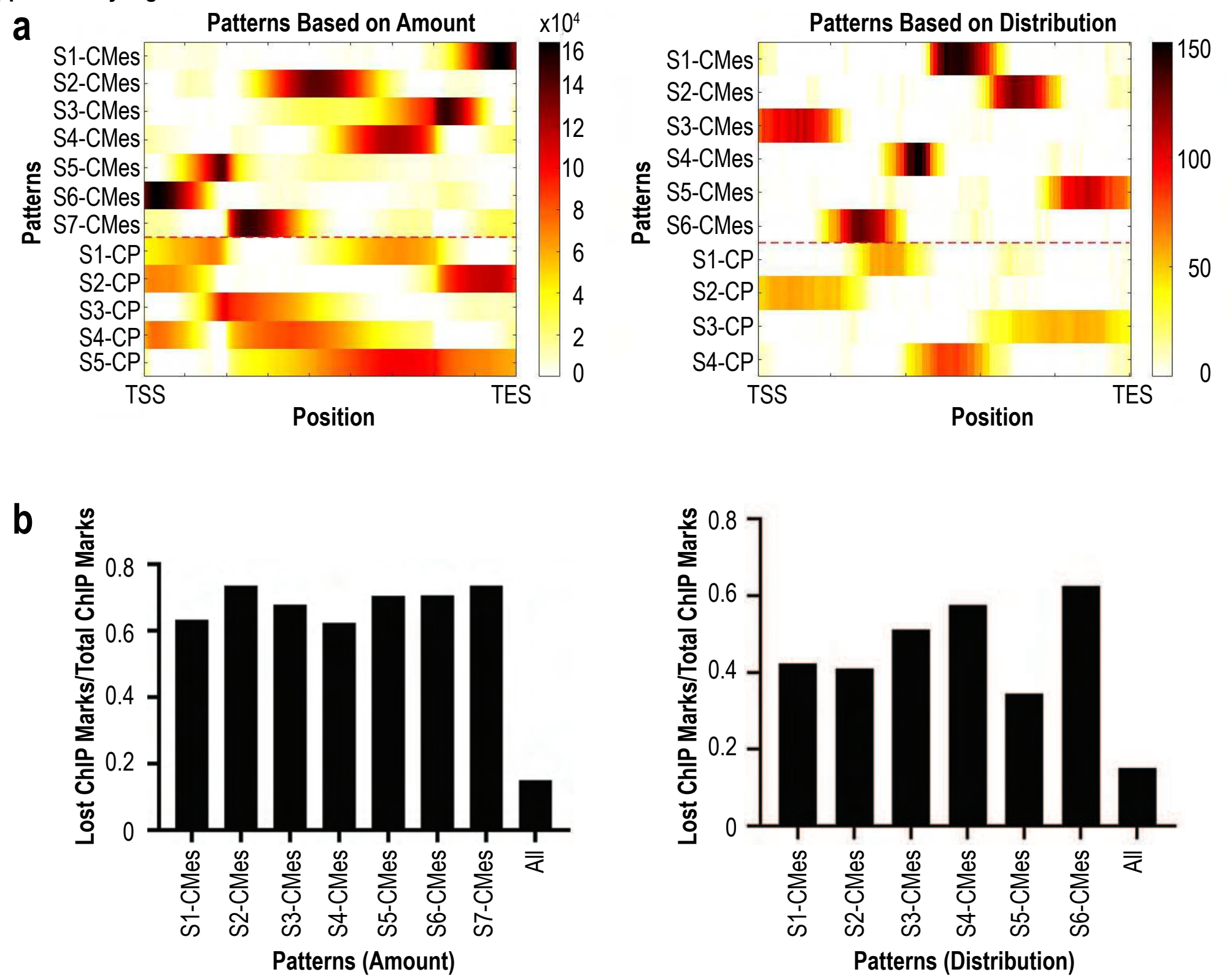

C 250
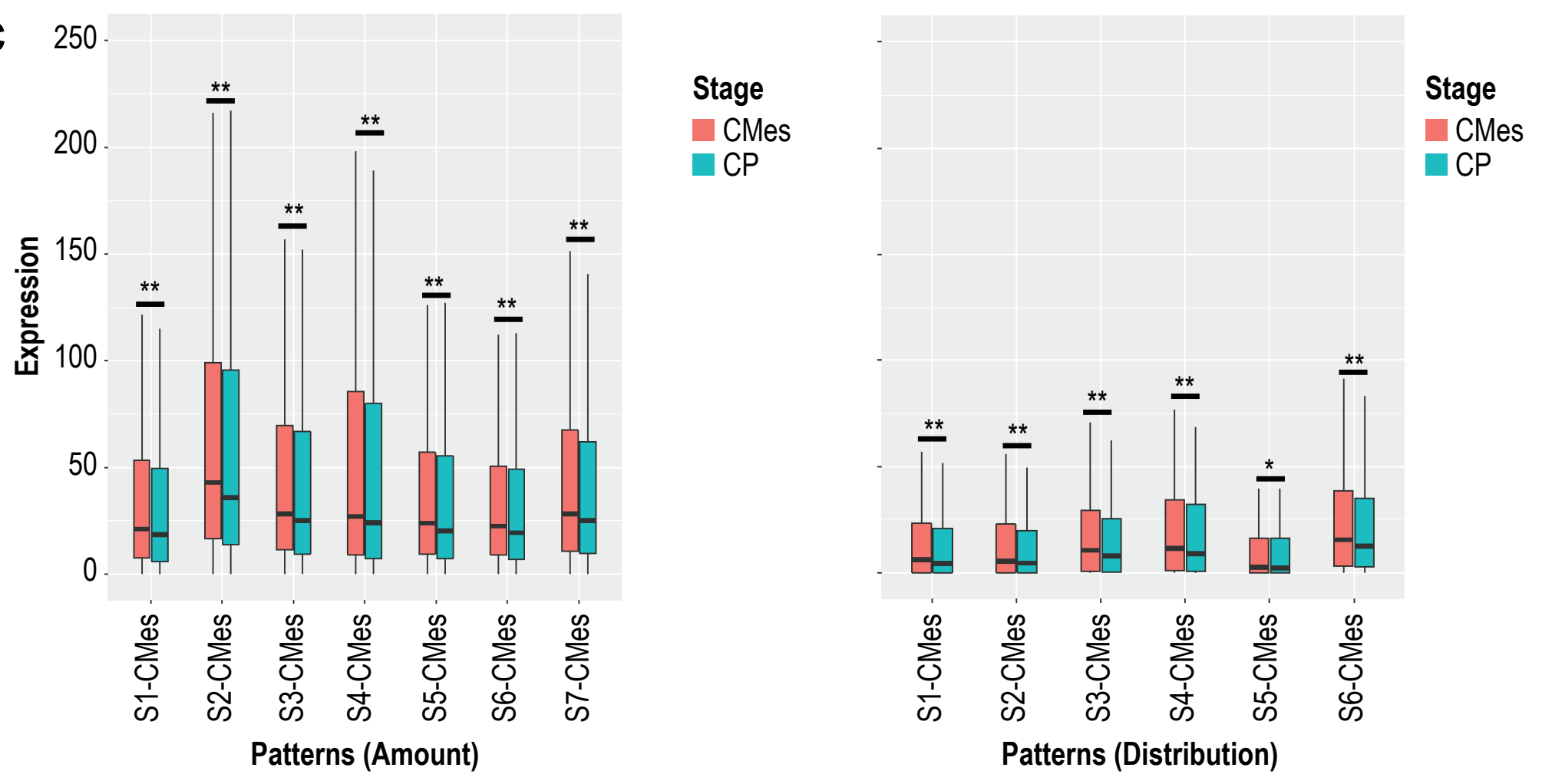
980 Supplementary Figure 4: Localized regions of H2Bub1 in the cardiac mesoderm stage

981 predict decreased expression in the cardiac progenitor stage

982 a) Patterns identified in the H2Bub1 peaks in cardiac mesoderm (CMes) and in cardiac

983 progenitor (CP). The graphs depict the H2Bub1 occupancy in the clusters of genes, called

984 patterns, over the gene body. Heat maps showing patterns based on amount are in the left

985 panel, and based on distribution are shown in the right panel. A pattern is defined as a group

986 of genes that have a similar structure to the distribution or amount of H2Bub1 across the

987 gene body.

988 b) Ratio of H2Bub1 regions lost to total H2Bub1 regions in each of the patterns (based on

989 amount in the left panel and based on distribution in the right panel) and the average ratio

990 (labeled all) between CMes and CP.

991 c) Expression levels from the RNA-seq data of the genes in each pattern (based on amount in the

992 left panel and based on distribution in the right panel) in CMes (red) and CP stages (blue).

993 Data are shown as a box plot, where the center line is the median, the box limits are the

994 upper and lower quartiles, and the whiskers are $1.5 \mathrm{x}$ the interquartile range. Paired, one-sided

995 Wilcoxon Rank Sum Test, * $\mathrm{p}<0.05, * * \mathrm{p}<0.01$. 


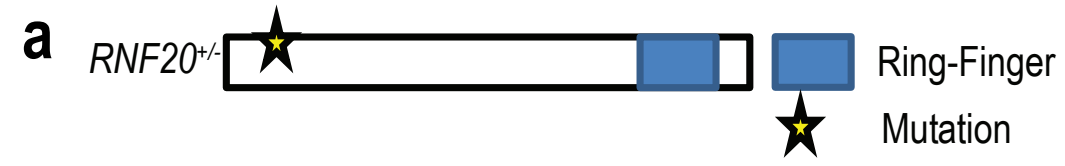

Wild Type

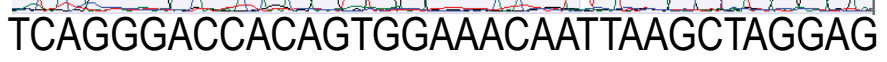
RNF20+-1

b

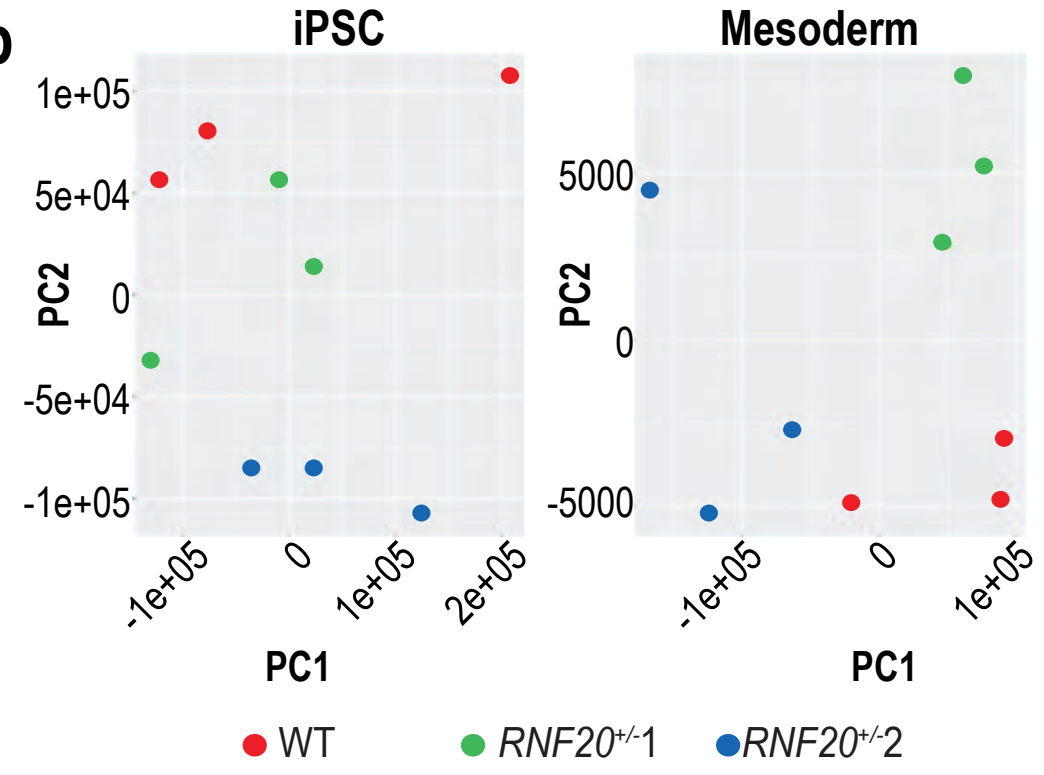

d More $R$ NF20+-1 H2Bub1 in mutant mesoderm

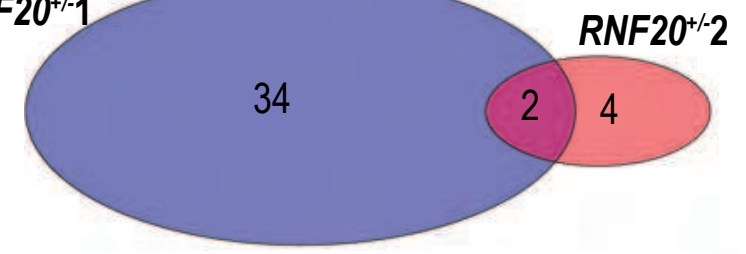

TCAGGGACCACA . . . . . . . . . . . GGAGG TCAGGGACCACAGTGGAAACAA - ---GCTAGGAGG

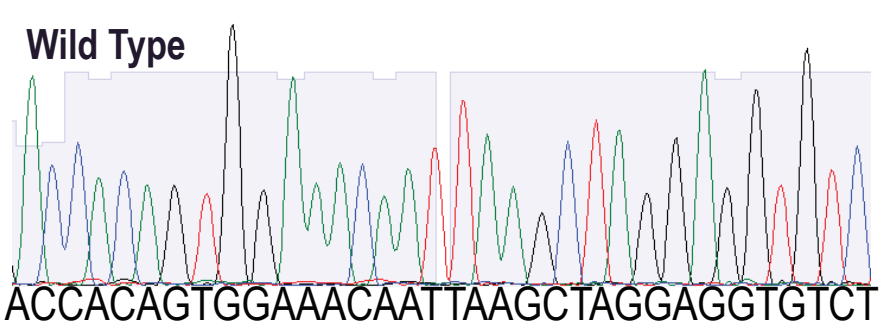
RNF20+-2

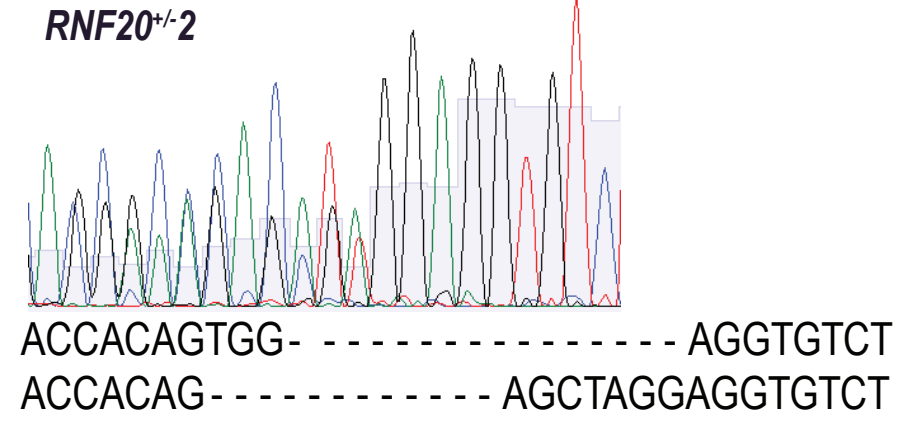
ACCACAG - . . . . . . . AGCTAGGAGGTGTCT
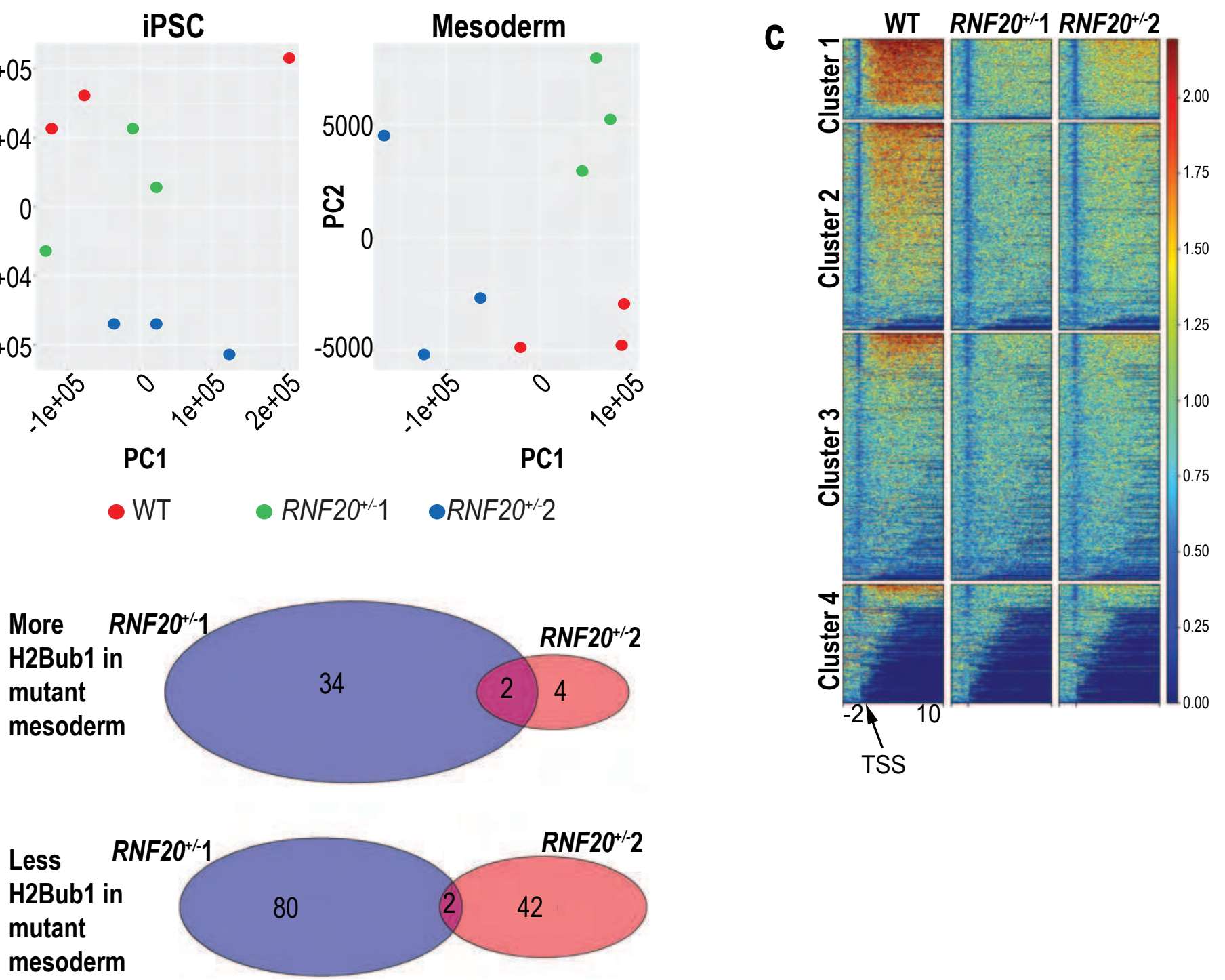


\section{Supplementary Figure 5: Characteristics of the $\mathrm{RNF}^{+\mathrm{O}^{+/}}$mutants}

997 a) Diagram of the RNF20 protein with its domains (Ring-Finger domain is shown in blue) and 998 where the created RNF20 mutations are located (with a star). Lower four panels are 999 sequencing traces of the wild-type and two independent $R N F 20^{+/}$iPSC lines demonstrating 1000 CRISPR-generated mutations.

1001 b) PCA plots of RNA-seq experiments comparing wild-type (red) and both $R N F 20^{+/}$mutants 1002 (green and blue) at iPSC and mesoderm (M) stages $(\mathrm{n}=3)$.

1003 c) H2Bub1 surrounding the transcriptional start site (TSS) $(-2 \mathrm{~Kb},+10 \mathrm{~Kb})$ in the wild-type and $1004 R N F 20^{+-}$mutants at the M stage. Genes are grouped by H2Bub1 levels into four clusters.

1005 The average profile, depicted using fold enrichment against random distribution values, 1006 across this region for each cluster in each stage is shown. This represents the average of three 1007 replicates.

1008 d) Regions with differential H2Bub1 in each $R N F 20^{+/}$mutant compared to wild-type at the $\mathrm{M}$ 1009 stage. These Venn Diagrams depict the overlap between the regions that have increased $1010 \mathrm{H} 2 \mathrm{Bub} 1$ in $R N F 20^{+/}$mutants compared to wild-type and the regions that have decreased $1011 \mathrm{H} 2 \mathrm{Bub} 1$ in both $R N F 20^{+/-}$mutants compared to wild-type. $R N F 20^{+/}$line 1 is in blue, $1012 \quad R N F 20^{+/}$line 2 is in red. 
a

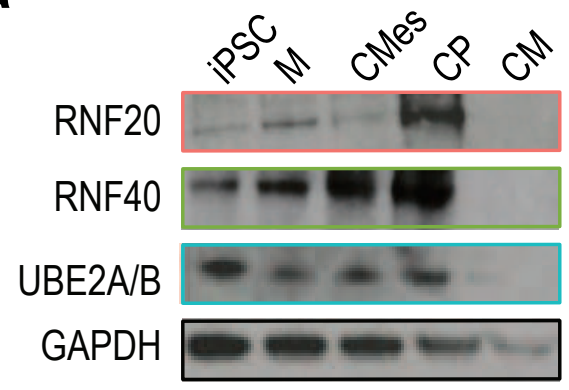

b Stage iPSC IPSC iPSC M M M

UBE2B +/+ $\quad-/-1 \quad-/-2 \quad+/+\quad-/-1 \quad-/-2$

RNF20

RNF40

UBE2A/B

GAPDH
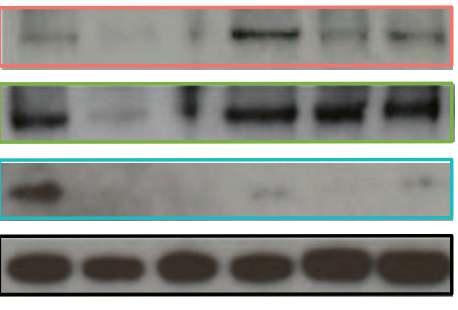

\section{5}

Rep 3

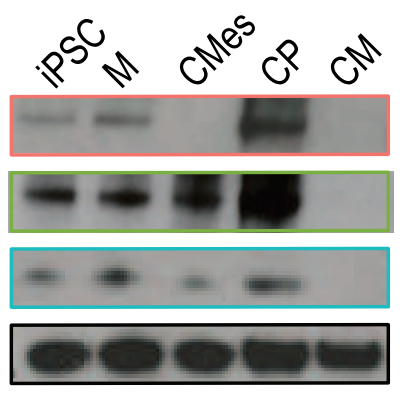

iPSC IPSC IPSC M M M

$+/+\quad-/-1 \quad-/-2 \quad+/+\quad-/-1 \quad-/-2$

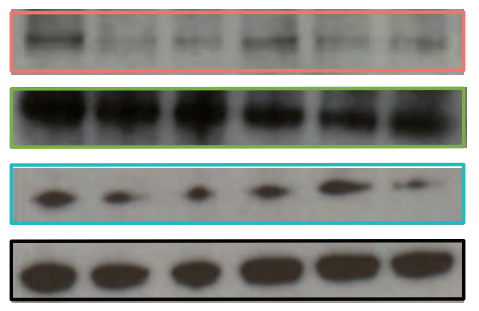

iPSC IPSC IPSC M M M

$+/+\quad-/-1 \quad-/-2 \quad+/+\quad-/-1 \quad-/-2$

RNF20 +/+ -/-1 -//2 +/+ -/-1 -//2

RNF20

RNF40

UBE2A/B

GAPDH
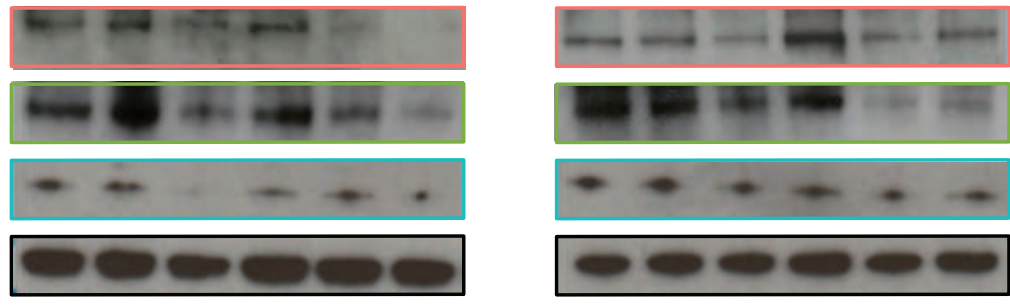

C Stage IPSC IPSC IPSC M M M UBE2B +/+ $\quad-/-1 \quad-/-2 \quad+/+\quad-/-1 \quad-/-2$

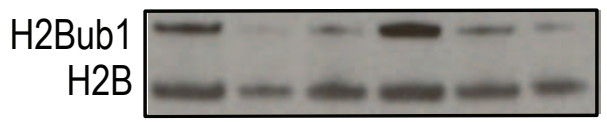

H2Bub1

$\mathrm{H} 2 \mathrm{~B}$

H2Bub1

$\mathrm{H} 2 \mathrm{~B}$

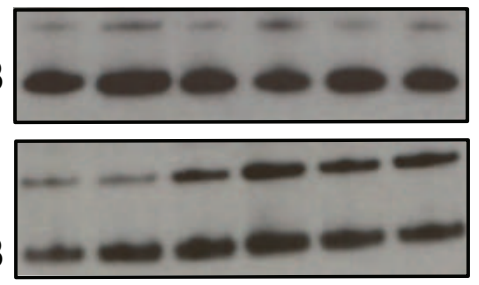

e Stage iPSC iPSC iPSC M M M RNF20 +/+ $\quad-/-1 \quad-/-2$ +/+ $\quad-/-1 \quad-/-2$ H2Bub1

$\mathrm{H} 2 \mathrm{~B}$

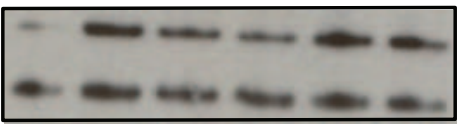

H2Bub1

$\mathrm{H} 2 \mathrm{~B}$

H2Bub1

$\mathrm{H} 2 \mathrm{~B}$

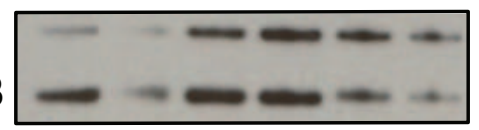

g RNF20 fl/- fl/t fl/-

Nkx Cre - ++

H2Bub1

$\mathrm{H} 2 \mathrm{~B}$
RNF40

- UBE2B

-WAC

- WT

-- RNF20+-2 


\section{Supplementary Figure 6: Analysis of RNF20-complex components and H2Bub1}

1014 a) Two additional replicates of western blots (see Figure 4b) showing RNF20-complex

1015 components changing during CM differentiation. Western blot for RNF20, RNF40, and

1016 UBE2B in five stages of CM differentiation (iPSC, mesoderm (M), cardiac mesoderm

1017 (CMes), cardiac progenitor (CP), and cardiomyocyte (CM)). The loading control is GAPDH.

1018 b) Two additional western blot replicates (see Figure 4e) depicting RNF20-complex components

1019 (RNF20, RNF40, and UBE2B) in iPSC and M stages comparing wild-type and $U B E 2 B^{-1-}$

1020 cells. The loading control is GAPDH.

1021 c) Three western blot replicates (See Figure $4 \mathrm{f}$ for the quantification) depicting H2Bub1 in iPSC

1022 and $\mathrm{M}$ stages comparing wild-type and $U B E 2 B^{--}$cells. The loading control is $\mathrm{H} 2 \mathrm{~B}$.

1023 d) Two additional western blot replicates (see Figure 4i) depicting RNF20-complex components

1024 (RNF20, RNF40, and UBE2B) in iPSC and M stages comparing wild-type and $R N F 20^{+-}$

1025 cells. The loading control is GAPDH.

1026 e) Three western blot replicates (See Figure $4 \mathrm{j}$ for the quantification) depicting H2Bub1 in iPSC

1027 and M stages comparing wild-type and $R N F 2 O^{+-}$cells. The loading control is $\mathrm{H} 2 \mathrm{~B}$.

1028 f) RNA-seq data for RNF20-complex components (RNF2O, RNF40, UBE2B, and WAC) in wild-

1029 type and $R N F 20^{+/} 2$ mutant iPSC and M cells. Data are shown as mean $\pm \operatorname{SEM}(\mathrm{n}=3)$.

1030 g) One western blot replicate (See Figure $4 \mathrm{~m}$ for the quantification) depicting H2Bub1 in e12.25

1031 wild-type $\left(\operatorname{Rnf} 20^{\mathrm{fl} /-}:: N k x 2.5 \mathrm{Cre}^{-}\right.$and $\left.\operatorname{Rnf} 20^{\mathrm{fl} /+}:: N k x 2.5 \mathrm{Cre}^{+}\right)$and mutant $\left(\operatorname{Rnf} 20^{\mathrm{fl} /-}\right.$

$\left.1032 \quad:: N k x 2.5 \mathrm{Cre}^{+}\right)$mouse hearts. The loading control is $\mathrm{H} 2 \mathrm{~B}$. 


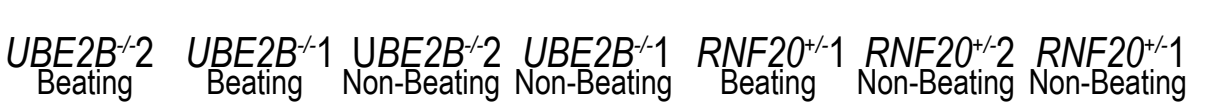
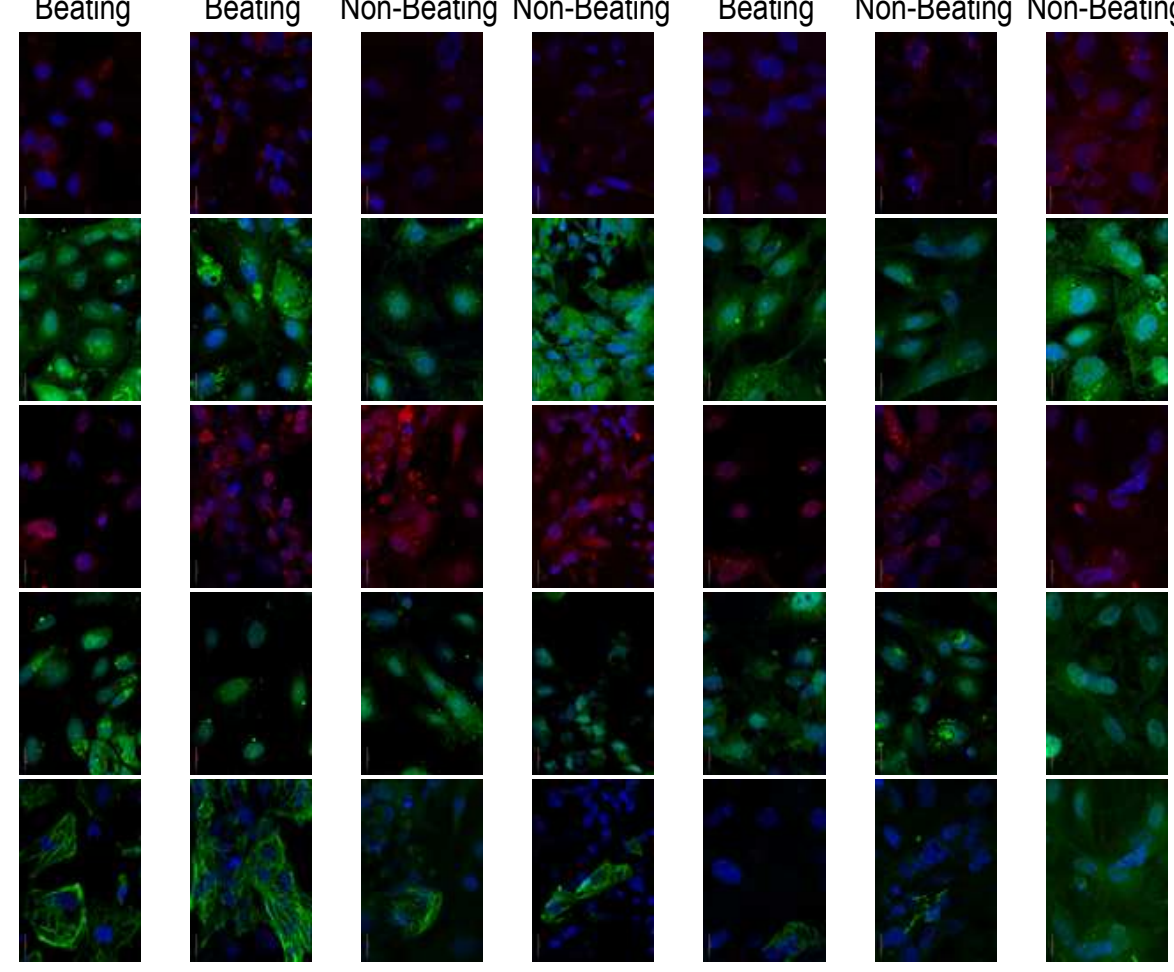

$\underset{\text { Beating }}{W T}$

?
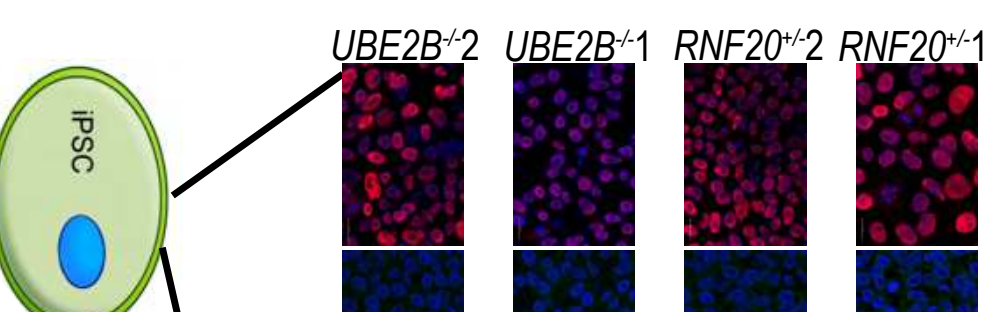

,
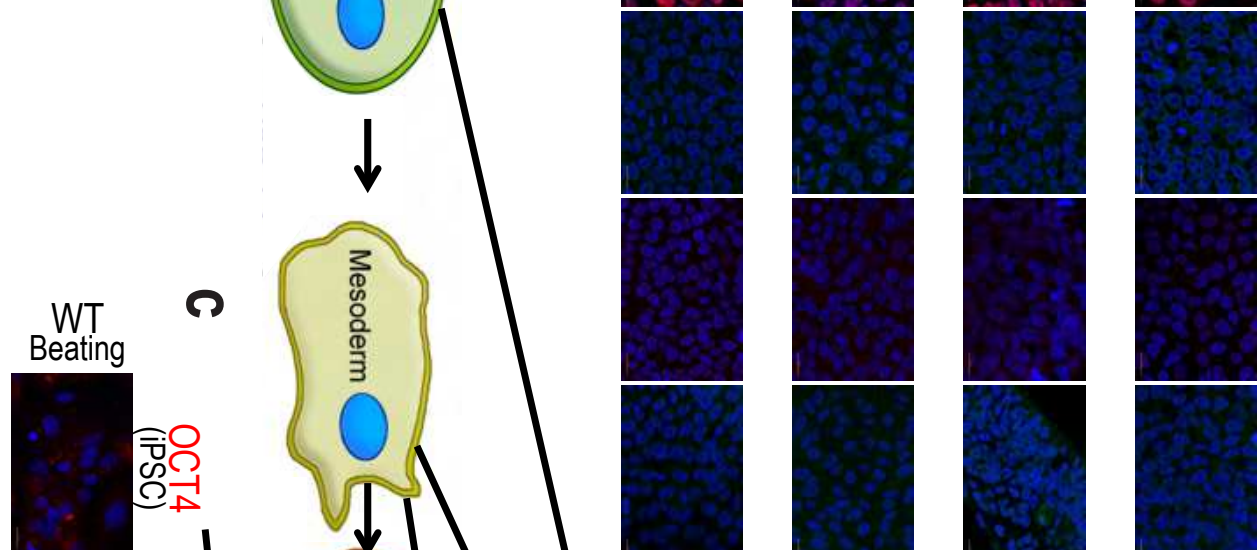

(1)
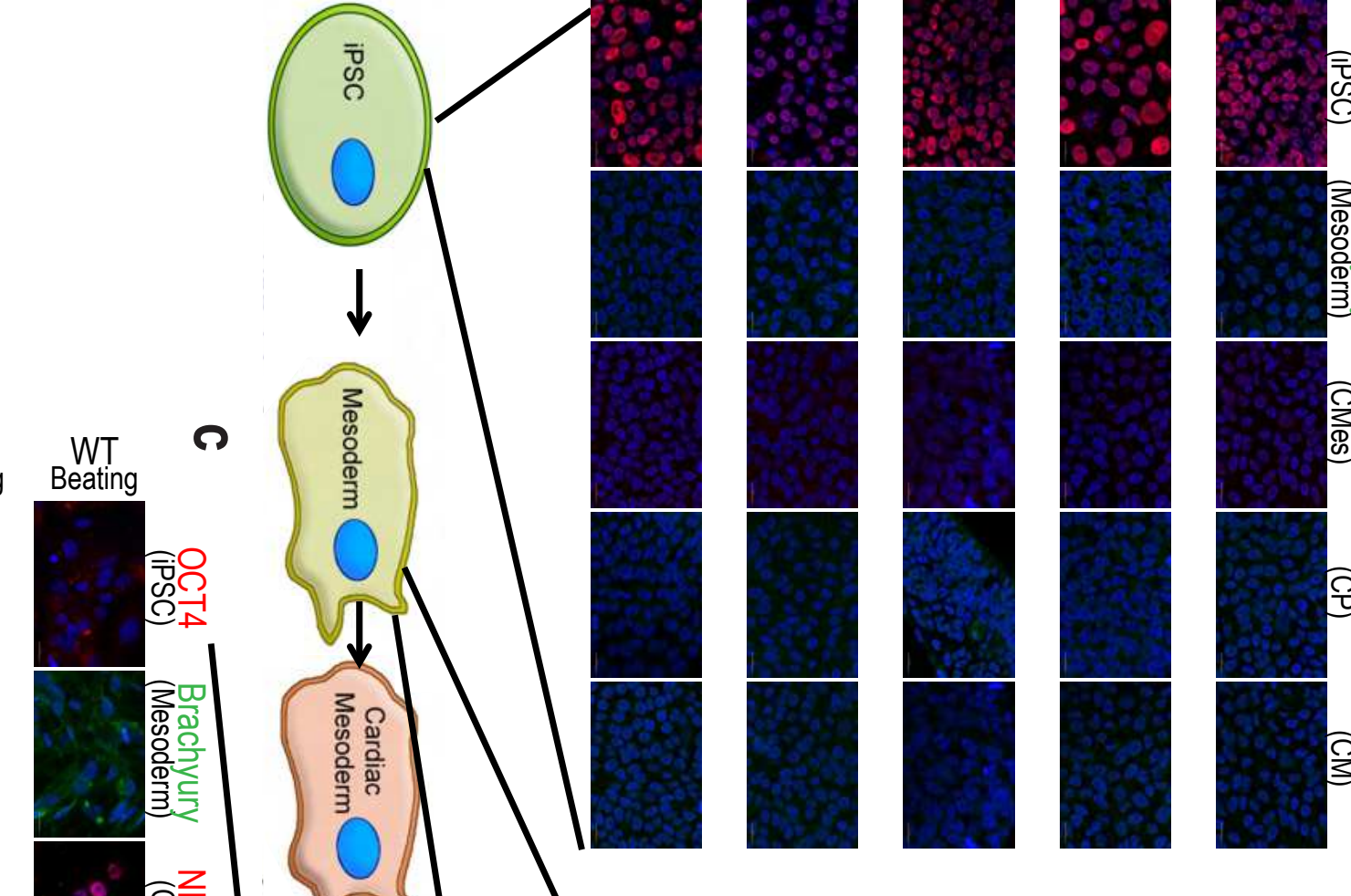

잉

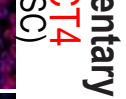

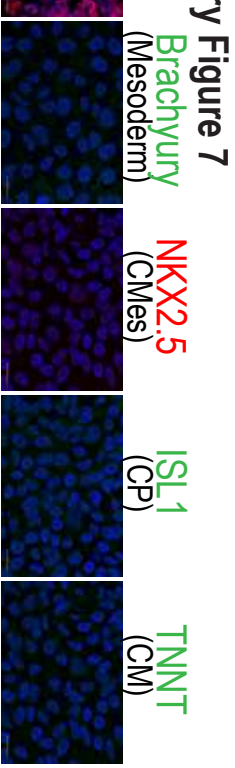

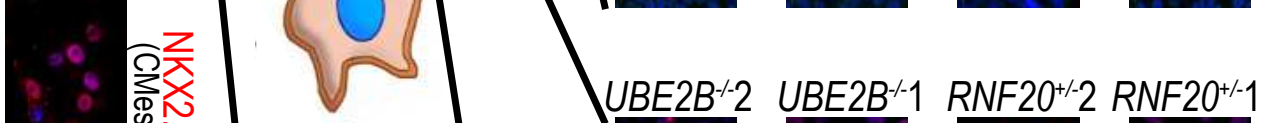
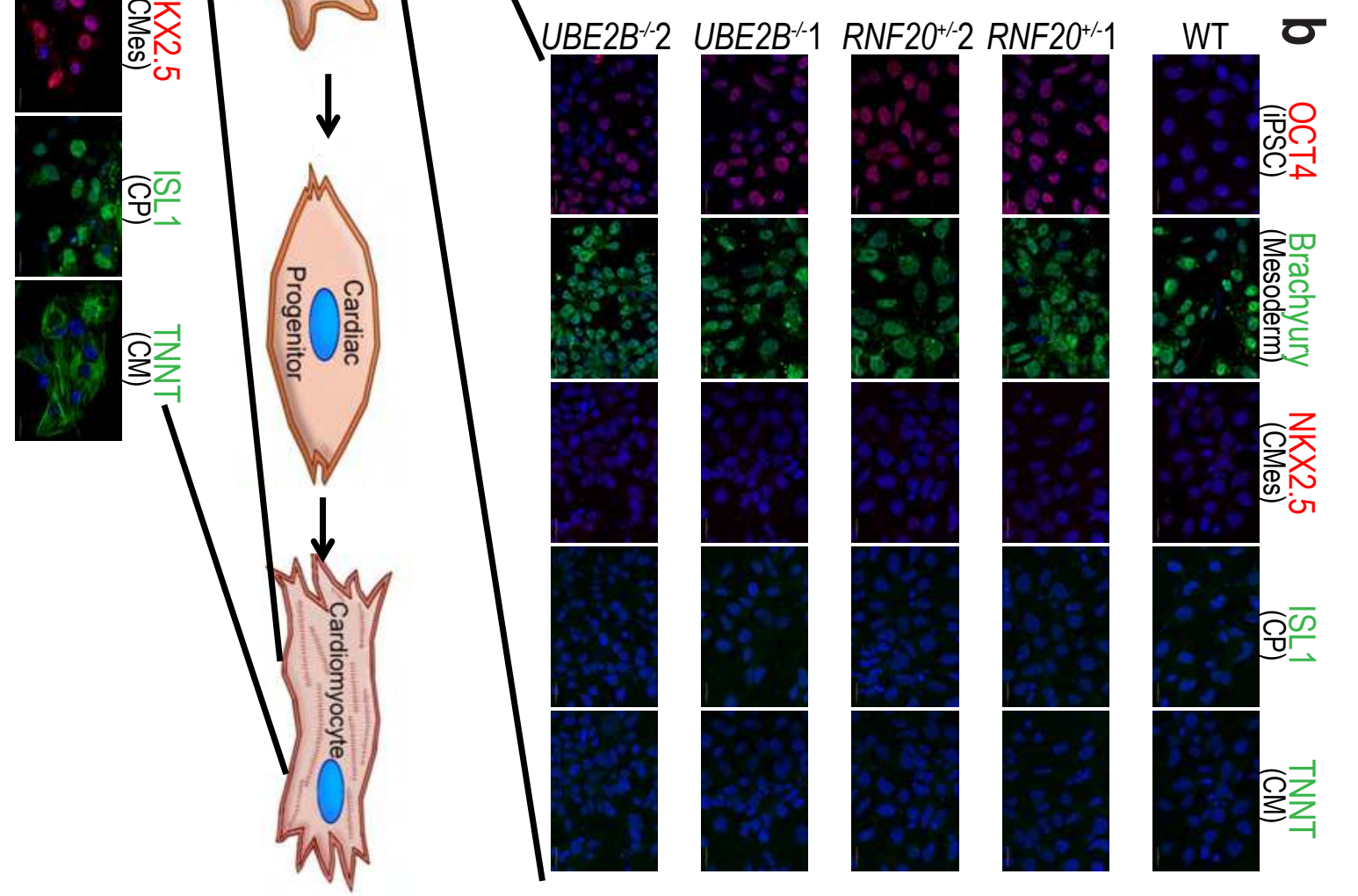


\section{Supplementary Figure 7: Differentiation trajectory for RN20 complex mutants}

1034 a) Immunofluorescence staining for marker genes for each of five stages in CM differentiation

1035 (iPSC: OCT4 (red), mesoderm (M): Brachyury (green), cardiac mesoderm (CMes): NKX2.5

1036 (red), cardiac progenitor (CP): ISL1 (green), and cardiomyocyte (CM): TNNT (green)) in

1037 each of the mutants (wild-type, $R N F 20^{+/} 1, R N F 20^{+/} 2, U B E 2 B^{-/} 1$, and $\mathrm{UBE}^{-1} \mathrm{~B}^{-/} 2$ ) at the

1038 iPSC stage.

1039 b) Immunofluorescence staining for marker genes for each of five stages in CM differentiation

1040 (iPSC: OCT4 (red), M: Brachyury (green), CMes: NKX2.5 (red), CP: ISL1 (green), and CM:

1041 TNNT (green)) in each of the mutants (wild-type, $R N F 20^{+-} 1, R N F 20^{+-} 2, U B E 2 B^{-/} 1$, and

$\left.1042 \quad \mathrm{UBE}_{2} \mathrm{~B}^{-} / 2\right)$ at the $\mathrm{M}$ stage.

1043 c) Immunofluorescence staining for marker genes for each of five stages in CM differentiation

1044 (iPSC: OCT4 (red), M: Brachyury (green), CMes: NKX2.5 (red), CP: ISL1 (green), and CM:

1045 TNNT (green)) in each of the mutants (wild-type, $R N F 20^{+-} 1, R N F 20^{+-} 2, U B E 2 B^{-/} 1$, and

$1046 \mathrm{UBE}^{-1-2}$ ) at the CM stage. Since these mutants form beating CMs some of the time,

1047 staining was done for beating and non-beating samples for each genotype, except $R N F 2 O^{+/} 2$

1048 since it never forms beating CMs. 


\section{Supplementary Figure 8}

a
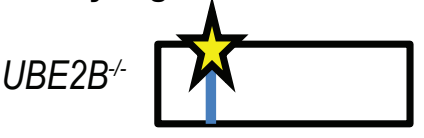

Catalytic Site

Mutation

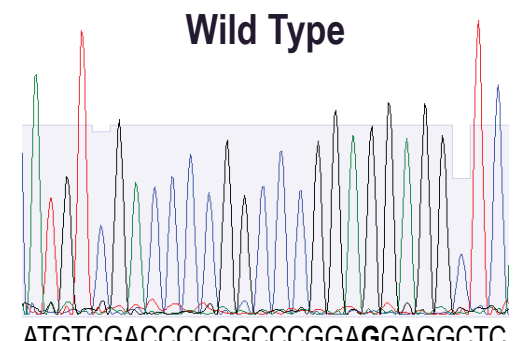

ATGTCGACCCCGGCCCGGAGGAGGCTC
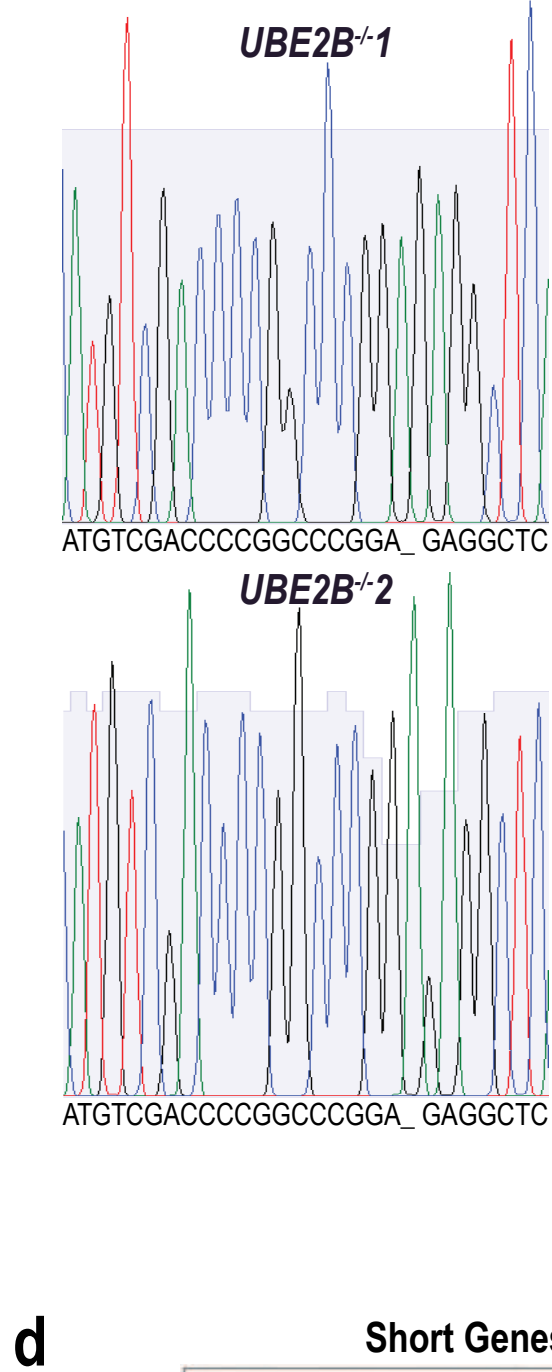

d

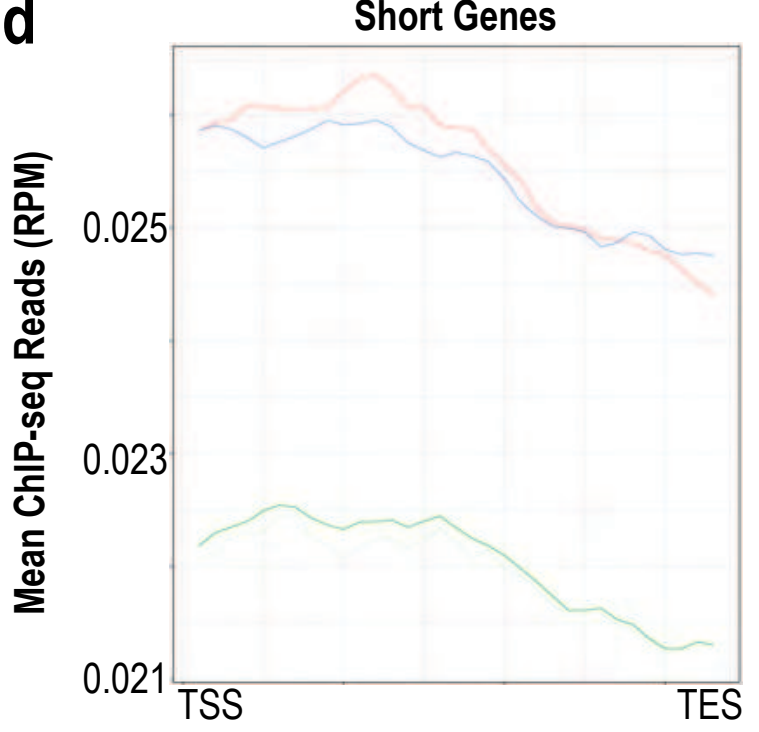

b

Day 30

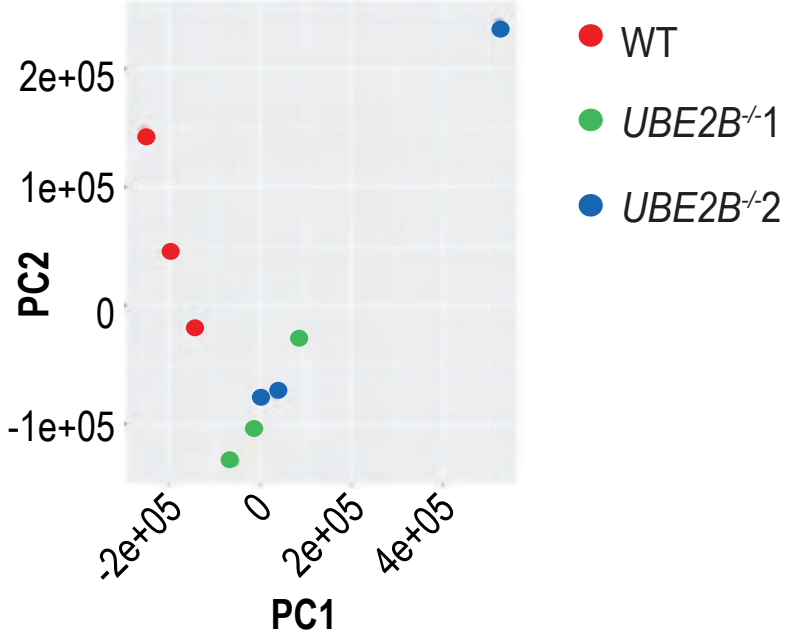

C

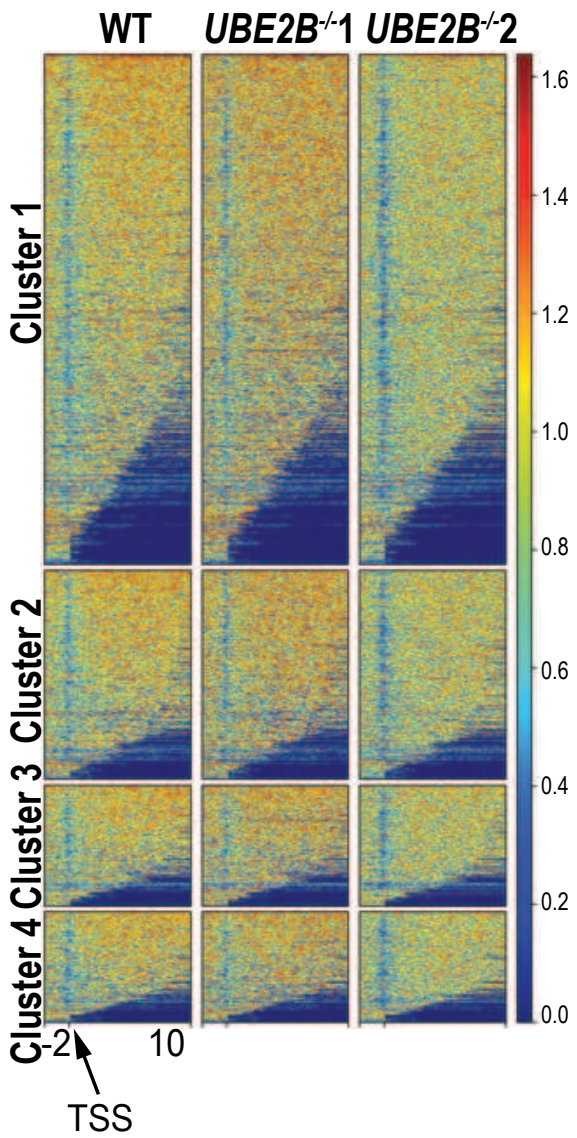

e

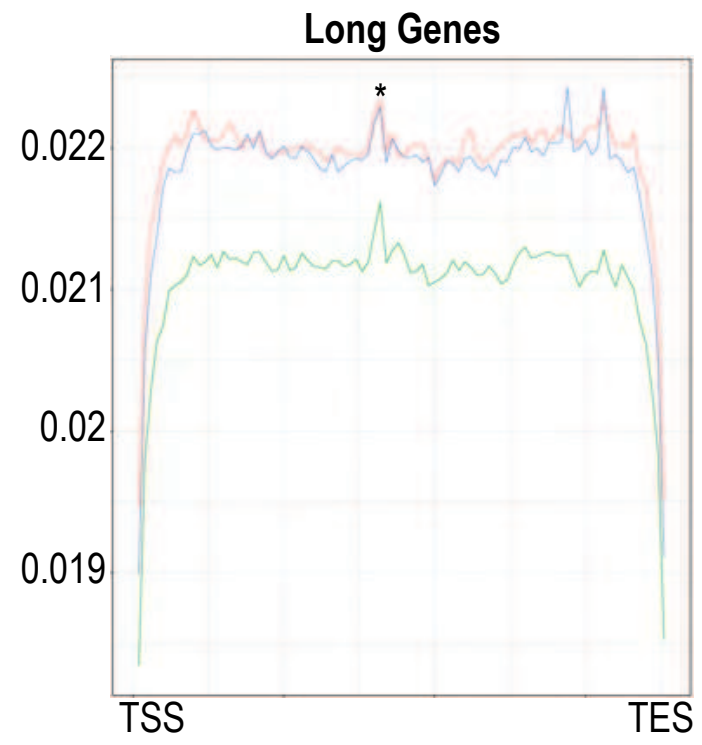




\section{Supplementary Figure 8: Characteristics of the $\boldsymbol{U B E 2 B ^ { - / - }}$ mutant}

1050 a) Diagram of the UBE2B protein with its catalytic site, shown in blue, and where the created $1051 U B E 2 B$ mutations are located (with a star). Lower three panels are sequencing traces of the 1052 wild-type and two independent $U B E 2 B^{-/}$iPSC lines demonstrating CRISPR-generated 1053 mutations.

1054 b) PCA plots of RNA-seq experiments comparing wild-type (red) and both $U B E 2 B^{-/}$mutants 1055 (green and blue) at the cardiomyocyte stage $(\mathrm{n}=3)$.

1056 c) H2Bub1 surrounding the transcriptional start site (TSS) $(-2 \mathrm{~Kb},+10 \mathrm{~Kb})$ in the wild-type and $1057 U B E 2 B^{-/}$mutants at the cardiomyocyte stage. Genes are grouped by H2Bub1 levels into four 1058 clusters. The average profile, depicted using fold enrichment against random distribution 1059 values, across this region for each cluster in each stage is shown. This represents the average 1060 of three replicates.

1061 d) Metagene plot for H2Bub1 levels in wild-type (blue) and $U B E 2 B^{-/}$mutants (green and red) 1062 across all of the short genes (less than $100 \mathrm{bp})(\mathrm{n}=3)$.

1063 e) Metagene plot for H2Bub1 levels in wild-type (blue) and $U B E 2 B^{-/}$mutants (green and red) 1064 across all of the long genes (greater than $10 \mathrm{~Kb})(\mathrm{n}=3)$. The * indicates the accumulation 1065 near the center of the gene. 
1066 Supplemental Table 1: Primers A list of all of the primers used in this study and their

1067 application.

\begin{tabular}{|c|c|c|}
\hline Primer ame & Se uence & pplication \\
\hline $18 s$ & CTT & Mouse Calcium RT-PCR \\
\hline $18 s \mathrm{R}$ & $\begin{array}{llll}\mathrm{CC} & \mathrm{TC} & \mathrm{T} & \mathrm{T}\end{array}$ & Mouse Calcium RT-PCR \\
\hline Cacnalc & TTC TTTC & Mouse Calcium RT-PCR \\
\hline CacnalcR & TT CCTCC TC & Mouse Calcium RT-PCR \\
\hline$J c x$ & $\begin{array}{llllll}\mathrm{TC} & \mathrm{C} & \mathrm{TCT} & \mathrm{C} & \mathrm{T} & \mathrm{T}\end{array}$ & ouse Calcium RT-PCR \\
\hline & $\begin{array}{llll}\text { СТCC } & \text { C } & \text { CTCC } & \text { C }\end{array}$ & ouse Calcium RT-PCR \\
\hline$y r^{2}$ & CCTT CCT $\quad$ T $\mathrm{C}$ & ouse Calcium RT-PCR \\
\hline$v r 2 \mathrm{R}$ & TT $\quad$ T TC $\quad \mathrm{C}$ & Iouse Calcium RT-PCR \\
\hline erca2a & $\mathrm{T} \mathrm{T} \mathrm{TC}$ & Iouse Calcium RT-PCR \\
\hline $\operatorname{serca} 2 a \mathrm{R}$ & CTTT TC CC & Mouse Calcium RT-PCR xpe \\
\hline SAPDH xon & $\begin{array}{llll}\mathrm{T} & \mathrm{T} & \mathrm{TTC} \\
\end{array}$ & uman Control or 2 u 1 ChIP-se \\
\hline JAPDH & C CCT & uman Control or 2 u $1 \mathrm{Ch}$ \\
\hline GAPDH Promoter & $\mathrm{C}$ TC $\mathrm{TC}$ & uman Control or \\
\hline JAPDH Promoter R & T $\quad$ CTTCCC TTC & uman Control or $2 \mathrm{u} 1$ ChIP-se \\
\hline RNF20 & C CT TCTTCTTCT CC & enot ping iPSC Mutants \\
\hline $2 N F 20 \mathrm{R}$ & СС ССТТССС ТTCTC & enot ping iPSC Mutants \\
\hline$R N F 20 \mathrm{Se}$ uence & СТTCССC Т CTCC & enot ping iPSC Mutants \\
\hline$U B E 2 B$ & $\begin{array}{llllll}\text { C } & \text { TC } & \text { TT } & \text { C } & \text { TT } & \text { TTT }\end{array}$ & enot ping iPSC Mutants \\
\hline$U B E 2 B \mathrm{R}$ & $\begin{array}{llll}\mathrm{C} & \mathrm{C} & \mathrm{CTT} & \mathrm{T}\end{array}$ & enot ping iPSC Mutants \\
\hline$U B E 2 B$ Se uence & СССТT CC СТT & enot ping iPSC Mutants \\
\hline $\operatorname{Rnf} 20^{+/} \quad$ ull allele & $\begin{array}{lllll}\text { CC } & \text { TT } & \text { CC } & \text { TT } & \text { TCT }\end{array}$ & Mouse Mouse genot ping \\
\hline $\mathrm{T}$ allele & ССТTC ССТC & Mouse Mouse genot ping \\
\hline $\operatorname{Rnf} 20^{+/} \mathrm{R}$ & СТC СТ Т ТTCTCTC CT CT & Mouse Mouse genot ping \\
\hline $\operatorname{Rnf} 20^{+/-} \mathrm{R}$ & TCСТC CT T ТTCТСТC CT CT & Mouse Mouse genot ping \\
\hline$U B E 2 B$ & $\begin{array}{lllllll}\mathrm{C} & \mathrm{CT} & \mathrm{C} & \mathrm{C} & \mathrm{T} & \mathrm{TC}\end{array}$ & uman Se uencing c \\
\hline$U B E 2 B \mathrm{R}$ & CC $\quad$ CTCTT & uman Se uencing $\mathrm{c}$ \\
\hline
\end{tabular}


1069 Supplemental Table 2: Antibodies A list of all of the antibodies used in this study, their

1070 species, their dilution, and their application.

\begin{tabular}{|c|c|c|c|c|}
\hline nti od & Catalogue & Species & ilution & pplication \\
\hline $2 \mathrm{u} 1$ & Cell Signaling $55 \quad \mathrm{~s}$ & $\mathrm{Ra}$ it & & ChIP \\
\hline $\operatorname{Igg}$ & Cell Signaling $22 \mathrm{~s}$ & $\mathrm{Ra}$ it & & ChIP \\
\hline rach ur & $\mathrm{R} \quad \mathrm{s}$ stems 2085-SP & oat & 120 & I cells \\
\hline Cardiac Troponin T & Invitrogen $M \quad 5-12 \quad 0$ & Mouse & 1200 & I cells \\
\hline IS 1 & S $\quad 3.5$ supernatant & Rat & 110 & I cells \\
\hline 2.5 & Santa Cru sc- 8 & oat & 1200 & I cells \\
\hline CT- a & Cell Signaling $280 \mathrm{~S}$ & $\mathrm{Ra}$ it & 1200 & I cells \\
\hline C 31 & iosciences 5502 & Rat & 1500 & I sections \\
\hline 2.5 & Santa Cru sc- 8 & oat & 1100 & I sections \\
\hline $\mathrm{R} \quad 20$ & Cell Signaling 11 & $\mathrm{Ra}$ it & 1200 & I sections \\
\hline $\mathrm{T} 1$ & ovus $\quad-\mathrm{h} 2$ & Mouse & 1250 & I sections \\
\hline alpha-tu ulin & Invitrogen 220 & Mouse & 11000 & estern \\
\hline $\mathrm{P}$ & Invitrogen $\mathrm{M} \quad 51538 \mathrm{RP}$ & Mouse & 120000 & estern \\
\hline 2 & Millipore $0-3 \quad 1$ & $\mathrm{Ra}$ it & 112000 & estern \\
\hline $2 \mathrm{u} 1$ & Cell Signaling $55 \mathrm{~s}$ & $\mathrm{Ra}$ it & 1200000 & estern \\
\hline $\mathrm{R} \quad 20$ & Cell Signaling 11 & $\mathrm{Ra}$ & 1500 & estern \\
\hline 0 & cam a 1130 & $\mathrm{Ra}$ & 11000 & estern \\
\hline 2 & enTex $\quad \mathrm{T} \quad 100 \quad 1$ & $\mathrm{Ra}$ & 1500 & estern \\
\hline
\end{tabular}

1071 


\section{Movie Legends}

1073 Movie 1: Movie shows wild-type cardiomyocytes under bright-field microscopy.

1074

1075 Movie 2: Movie shows $R N F 20^{+/} 1$ cardiomyocytes beating under bright-field microscopy.

1076

1077 Movie 3: Movie shows $R N F 20^{+/-} 1$ cardiomyocytes not beating under bright-field microscopy.

1078

1079 Movie 4: Movie shows $R N F 20^{+/-} 2$ cardiomyocytes not beating under bright-field microscopy.

1080

1081 Movie 5: Movie shows $U B E 2 B^{-/} 1$ cardiomyocytes beating under bright-field microscopy.

1082

1083 Movie 6: Movie shows $U B E 2 B^{-/} 1$ cardiomyocytes not beating under bright-field microscopy.

1084

1085 Movie 7: Movie shows $U B E 2 B^{-/ 2}$ cardiomyocytes beating under bright-field microscopy.

1086

1087 Movie 8: Movie shows $U B E 2 B^{-/} 2$ cardiomyocytes not beating under bright-field microscopy. 


\section{Supplemental Data 1: ChIP-seq wild-type iPSC-derived cardiomyocytes}

1089 Tab 1: contains the genes in Clusters 1-4 displayed in Fig. 2a and Fig. 5c.

1090 Tabs 2-21: contain the gene ontology terms for the genes in Clusters 1-4 in iPSC, mesoderm 1091 (M), cardiac mesoderm (CMes), cardiac progenitor (CP), and cardiomyocyte (CM) in Fig. 2a. 1092 Tabs 22-29: contain the differentially occupied regions between all of the consecutive stages 1093 (iPSC v. M, M v. CMes, CMes v. CP, and CP v. CM) of CM differentiation (Fig. 2).

1094 Tabs 30-31: contain the genes in each pattern of each version (Fig. S4).

1095

1096 Supplemental Data 2: RNA-seq wild-type iPSC-derived cardiomyocytes

1097 Tabs 1-8: contain the genes that are differentially expressed in between all of the consecutive 1098 stages (iPSC v. mesoderm (M), M v. cardiac mesoderm (CMes), CMes v. cardiac progenitor 1099 (CP), and CP v. cardiomyocyte (CM)) of CM differentiation (Fig. S3).

1100 Tab 9: contains the RNA-seq quality metrics.

1101

1102 Supplemental Data 3: ChIP-seq RNF 20 $^{+/-}$iPSCs and mesoderm

1103 Tabs 1-4: contain the differentially occupied regions between wild-type and $R N F 20^{+/}$iPSCs 1104 (Fig. 5).

1105 Tab 5: contains the genes in Clusters 1-4 displayed in Fig. S5c.

1106 Tabs 6-9: contain the differentially occupied regions between wild-type and $R N F 20^{+-}$mesoderm 1107 (Fig. S5).

1108

1109

1110 


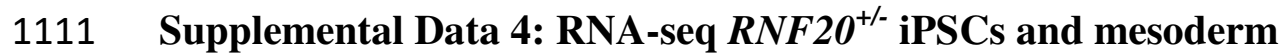

1112 Tabs 1-8: contain the genes that are differentially expressed in between wild-type and $R N F 20^{+/-}$

1113 iPSCs, and wild-type and $R N F 20^{+/-}$mesoderm (Fig. 5, S5).

1114 Tab 9: contains the RNA-seq quality metrics.

1115

1116 Supplemental Data 5: ChIP-seq $U$ BE2B ${ }^{-/-}$cardiomyocytes

1117 Tab 1: contains the genes in Clusters 1-4 displayed in Fig. S8c.

1118 Tabs 2-5: contain the differentially occupied regions between wild-type and $U B E 2 B^{-/-}$

1119 cardiomyocytes (Fig. S8).

1120 Tabs 6-10: contain the genes used for each metagene plot (Fig. 7).

1121

\section{Supplemental Data 6: RNA-seq $\boldsymbol{U B E} 2 \boldsymbol{B}^{-/-}$cardiomyocytes}

1123 Tabs 1-4: contain the genes that are differentially expressed in between wild-type and $U B E 2 B^{-/-}$

1124 cardiomyocytes (Fig. 6, S8).

1125 Tab 5: contains the RNA-seq quality metrics. 


\section{Online Methods}

\section{Mouse husbandry}

1128 The wild-type mouse data in Figure S1 are on the CD1 background. The Rnf2 $20^{+/}$, Rnf $20^{f l f l}$, and

1129 all of the cre lines used in this study are maintained on the C57BL/6 background. All cre mice

1130 used were validated for correct expression and penetrance by testing their offspring with a

1131 ROSA $^{\mathrm{mT} / \mathrm{mG}}$ mouse at e8.5 for GFP and tdTomato expression. This research complies with all

1132 ethical regulations; mouse experiments were performed in a manner approved by the Yale

1133 University Institutional Animal Care and Use Committee.

1134

\section{Cell lines and cardiomyocyte differentiation}

1136 PGP1 cells (gift from the Seidman lab) were maintained on matrigel with mTESR media.

1137 Differentiation was performed in accordance with previously published work ${ }^{37}$. Briefly, 0.5

1138 million cells (or 0.7 million cells in the $U B E 2 B^{-/-}$mutants), were plated on matrigel about four 1139 days prior to onset of differentiation. When the cells reached $100 \%$ confluency, differentiation

1140 was started using $12 \mu \mathrm{M}$ of CHIR99021 in RPMI media supplemented with B-27 minus insulin.

1141 Twenty-four hours later, the media was replaced with RPMI media supplemented with B-27

1142 minus insulin. Forty-eight hours later, the media was replaced with $5 \mu \mathrm{M}$ of IWP4 in RPMI

1143 media supplemented with B-27 minus insulin. Forty-eight hours later, the media was replaced 1144 with RPMI media supplemented with B-27 minus insulin. On day eight and every 2-3 days after 1145 that, the media was replaced with RPMI media supplemented with B-27. After the onset of 1146 beating, the cardiomyocytes (CMs) were selected using $4 \mathrm{mM}$ lactate in DMEM without glucose.

1147 A well was considered to be beating if two distinct regions on the plate contained beating cells. 
1148 Prior to the collection of samples, mycoplasma testing was performed on all cell lines using the

1149 MycoAlert Plus Mycoplasma Detection Kit from Lonza (LT07-703).

1151 Since lactate selection is a metabolic selection, it can only work if the cells are CMs. Therefore,

1152 if a well was not beating, no lactate selection was done and the subsequent analyses were done

1153 on the non-selected cells.

\section{CRISPR-Cas9 editing of human iPSCs}

1156 uides ere created using MIT s CRISPR tool (http://crispr.mit.edu/) to create mutations in

$1157 R N F 20$ and $U B E 2 B$ (2 each) near the transcriptional start site. These guides and the other

1158 necessary sequence were synthesized as gblocks and cloned using the TOPO TA cloning kit.

1159 Two different protocols were used to create knockouts. $U B E 2 B^{-/-} 1, R N F 20^{+-} 1$, and $R N F 2 O^{+/-} 2$

1160 were created using the Lonza Nucleofector 2b with the Human Stem Cell Nucleofector Kit 2

1161 according to the manu acturer s protocol. 1 million cells were nucleofected using program B020

1162 with $2 \mu \mathrm{g}$ of a plasmid containing Cas9 (PX459 Addgene) and $2 \mu \mathrm{g}$ of a plasmid containing the

1163 sgRNA. $U B E 2 B^{-/} 2$ was created by adding $10 \mu \mathrm{g}$ of Cas9 plasmid and $10 \mu \mathrm{g}$ of sgRNA plasmid

1164 to 1 million cells using the Bio-Rad Gene Pulser XCell Eukaryotic System with a homemade

1165 nucleofection solution $\left(5 \mathrm{mM} \mathrm{KCl}, 5 \mathrm{mM} \mathrm{MgCl}_{2}, 15 \mathrm{mM}\right.$ Hepes, $102.94 \mathrm{mM} \mathrm{Na}_{2} \mathrm{HPO}_{4}, 47.06$

$1166 \mathrm{mM} \mathrm{NaH}_{2} \mathrm{PO}_{4}$ monohydrate, $\mathrm{pH}=7.2$ ) according to previously published work. The following

1167 parameters were used: $250 \mathrm{~V}, 500 \mu \mathrm{F}$, and a $0.4 \mathrm{~cm}$ cuvette ${ }^{68}$. These cells grew for two days with

1168 mTESR supplemented with $5 \mu \mathrm{M}$ of Y27632. Then, selection for receiving the Cas9 plasmid

1169 occurred for 2 days using $400 \mu \mathrm{g}$ of puromycin. About a week after transfection, individual

1170 colonies were picked and screened for mutations using primers flanking the sgRNA 
1171 (Supplemental Table 1). If there was a possibility of a mixed colony, individual colonies got

1172 picked from that colony and these subcolonies got screened. This process was repeated until

1173 colonies were pure. Additionally, the DNA was cloned into TOPO TA cloning it $(R N F 20)$ or

1174 Zero Blunt TOPO Cloning Kit (UBE2B) and sequenced to verify the ratio of the two different

1175 alleles was 1:1.

1176

1177

\begin{tabular}{|l|l|l|}
\hline Guide & Sequence & Cell Line(s) Developed \\
\hline RNF20_1 & ACAGTGGAAACAATTAAGCT & $R N F 2 O^{+-1} 1$ and $R N F 2 O^{+-} 2$ \\
\hline RNF20_2 & GTGGAAACAATTAAGCTAGG & N/A \\
\hline UBE2B_1 & AATCCCGCATGAGCCTCCTC & $U B E 2 B^{-1-} 1$ \\
\hline UBE2B_2 & ATCCCGCATGAGCCTCCTCC & $U B E 2 B^{-1-2}$ \\
\hline
\end{tabular}

1178 Surrounding sequence to the $g R N A$

1179 TGTACAAAAAAGCAGGCTTTAAAGGAACCAATTCAGTCGACTGGATCCGGTACCAA

1180 GGTCGGGCAGGAAGAGGGCCTATTTCCCATGATTCCTTCATATTTGCATATACGATA

1181 CAAGGCTGTTAGAGAGATAATTAGAATTAATTTGACTGTAAACACAAAGATATTAGT

1182 ACAAAATACGTGACGTAGAAAGTAATAATTTCTTGGGTAGTTTGCAGTTTTAAAATT

1183 ATGTTTTAAAATGGACTATCATATGCTTACCGTAACTTGAAAGTATTTCGATTTCTTG

1184 GCTTTATATATCTTGTGGAAAGGACGAAACACC (Guide)

1185 GTTTTAGAGCTAGAAATAGCAAGTTAAAATAAGGCTAGTCCGTTATCAACTTGAAAA

1186 AGTGGCACCGAGTCGGTGCTTTTTTTCTAGACCCAGCTTTCTTGTACAAAGTTGGCAT

1187 TA

1188

1189 Immunofluorescence of mice

1190 Mouse embryos and hearts were fixed in 4\% PFA overnight. They were placed in 30\% sucrose

1191 until the tissue sank and then embedded in OCT. $12 \mu \mathrm{M}$ sections were cut. These slides were 
1192 allowed to defrost and dry out for 3 hours on a slide warmer. The sections were post-fixed for 10

1193 minutes with 0.2\% PFA, and then washed 4 times with PBS for 20 minutes. The sections were

1194 either blocked in 5\% BSA, 10\% Goat serum, or Fish Gelatin Blocking agent in PBT (PBS+ 0.2\%

1195 Triton X100). The samples were incubated with primary antibodies for either 4 hours at RT or

1196 overnight (Supplemental Table 2). The samples were washed four times in PBT for about 20

1197 minutes, incubated with the corresponding chromophore conjugated secondary antibody (1:500)

1198 for about 45 minutes, and then washed four times in PBT for about 20 minutes. The second to

1199 last wash contained 1:2000 Hoechst. The samples were mounted on coverslips in ProLong Gold

1200 (molecular probes). The samples were imaged on the Zeiss Observer Z1 equipped with Apotome

1201 optical interference imaging.

1202

\section{Immunofluorescence of cells}

1204 iPSCs and mesodermal (M) cells were passaged using accutase. $650 \mu \mathrm{L}$ of accutase was added to 1205 each well of a 6 well plate for $5-7$ minutes at $37^{\circ} \mathrm{C}$ until the cells started to come off from the 1206 plate. After spinning down at $1000 \mathrm{rpm}$ for 5 minutes, the cells were plated on coverslips. A

$12070.1 \% \mathrm{w} / \mathrm{v}$ solution of gelatin had been added to these coverslips overnight at $37^{\circ} \mathrm{C}$. The next 1208 morning, these coverslips were rinsed with PBS, a 1/400 solution of matrigel in media was added 1209 for 1 hour, rinsed with PBS, and then the passaged cells were added. The media the cells were 1210 grown in while they were on the coverslips was normal cell culture media for the respective 1211 stage with $5 \mu \mathrm{M}$ of Y27632.

1212

1213 CMs were passaged using Trypsin-EDTA (0.25\%). $2 \mathrm{~mL}$ of Trypsin was added to each well of a 12146 well plate for 5 minutes at room temperature. After spinning down at $1000 \mathrm{rpm}$ for 5 minutes, 
1215 the cells were plated on coverslips. A $0.1 \% \mathrm{w} / \mathrm{v}$ solution of gelatin had been added to these

1216 coverslips overnight at $37^{\circ} \mathrm{C}$. The next morning, these coverslips were rinsed with PBS, and then

1217 the passaged cells were added. These cells were grown in RPMI20 (RPMI with 20\% FBS) with 5 $1218 \mu \mathrm{M}$ of $\mathrm{Y} 27632$ as per previous work ${ }^{37}$.

1220 After 2 days of culturing the iPSC, M, or CMs on coverslips, they were fixed in 4\% PFA for 10 1221 minutes. Extreme caution was taken when adding and removing liquids to not cause the cells to 1222 come off the coverslips. The coverslips were rinsed 4 times for 20 minutes with PBS. The

1223 sections were either blocked in 5\% BSA or 10\% Goat serum in PBT (PBS+ 0.2\% Triton X100).

1224 The samples were incubated with primary antibodies for either 4 hours at RT or overnight

1225 (Supplemental Table 2). The samples were washed four times in PBT for about 20 minutes, 1226 incubated with the corresponding chromophore conjugated secondary antibody (1:500) for about

122745 minutes, and then washed four times in PBT for about 20 minutes. The second to last wash 1228 contained 1:2000 Hoechst. The coverslips were mounted on slides in ProLong Gold (molecular 1229 probes). The samples were imaged on the Zeiss Observer Z1 equipped with Apotome optical 1230 interference imaging.

\section{Histology}

1233 Whole embryos or adult hearts were fixed overnight in $4 \%$ buffered paraformaldehyde. The adult

1234 hearts were then cut in half sagittal sections. These embryos or half hearts were dehydrated,

1235 cleared, and embedded in paraffin wax. Transverse sections (embryonic sections were $10 \mu \mathrm{m}$

1236 thick and adult heart sections were 3-6 $\mu \mathrm{m}$ thick) were cut using a microtome and were stained

1237 with hematoxylin and eosin following standard protocols. Images were taken on a Zeiss Axiovert 
1238 microscope, using an Axiocam driven by Axiovision software. Measurements (heart size,

1239 interventricular septum length, and compact myocardium thickness) were made using image ${ }^{69}$.

1240

\section{Transmission electron microscopy}

1242 The embryos were dissected in 4\% PFA. A small hole was made in an atrium with a scalpel to

1243 allow the fixative to get inside the heart. Embryonic heart tissue was fixed in 2.5\%

1244 glutaraldehyde and $2 \%$ paraformaldehyde in $0.1 \mathrm{M}$ sodium cacodylate buffer, $\mathrm{pH} 7.4$ for 1 hour

1245 at room temperature and left at $4^{\circ} \mathrm{C}$ for two days. The samples were rinsed in buffer, then post

1246 fixed in $1 \%$ osmium tetroxide and en-bloc stained in $2 \%$ aqueous uranyl acetate for another hour.

1247 This was followed by rinsing in distilled water and dehydrating in an ethanol series, then resin

1248 infiltration in Embed 812 (Electron Microscopy Sciences) and baking overnight at $60^{\circ} \mathrm{C}$.

1249 Hardened blocks were cut using a Leica UltraCut UC7. $60 \mathrm{~nm}$ sections were collected on

1250 formvar/carbon coated grids and contrast stained using $2 \%$ uranyl acetate and lead citrate. The

1251 sections were viewed in an FEI Tencai Biotwin TEM at $80 \mathrm{Kv}$. Images were taken using Morada

1252 CCD and iTEM (Olympus) software.

1253

1254 Protein extractions

1255 Mouse hearts were dissected and the blood was removed by flushing with PBS. The hearts were

1256 flash frozen in liquid nitrogen and homogenized on Mikro Dismembrator II. Cells were removed

1257 from the plates using accutase (for iPSC and M cells) or Trypsin-EDTA 0.25\% (for CMs).

1258 Proteins were extracted using NE-PER Nuclear and Cytoplasmic extraction reagents (Thermo

1259 fisher scientific 78833) supplemented with proteinase inhibitors (Roche) according to 
manufacturer instructions. After the fractions were acquired, they were combined. The proteins

1261 were analyzed by western blotting.

1262

\section{Histone extractions}

1264 Histones were extracted from the pellets from the protein extractions using the total histone

1265 extraction kit (Epigentek) according to manufacturer instructions. The extracted histones were 1266 analyzed by western blotting.

\section{Western blots}

1269 The protein samples were evenly loaded onto 4-12\% gradient Bis-Tris gels (Invitrogen)

1270 (UBE2B) or 3-8\% Tris-Acetate gels (NuPAGE) (RNF20 and RNF40), transferred to PVDF

1271 membrane, blocked overnight in 5\% dry milk in TBST, and incubated with primary antibodies

1272 overnight rocking at $4^{\circ} \mathrm{C}$ (Supplemental Table 2), followed by washes in TBST for 1 hour,

1273 incubated with corresponding HRP-conjugated secondary antibodies (Abcam ab6721, 1:20,000)

1274 for 1 hour at RT, washed in TBST for 4.5 hours. Images were acquired using film and scanned in

1275 to be quantified using image ${ }^{69}$. For a loading control for the wild-type mice over time (Fig.

1276 S1e), we used a Mini-PROTEAN TGX Stain-Free precast gel (Bio-Rad). For a loading control

1277 for the iPSCs, we used GAPDH (Fig. 4, S6). For a loading control for the RNF20 mutant mice, 1278 we used alpha-tubulin (Fig. 4, S6).

1279

1280 The histone samples were evenly loaded onto 4-12\% gradient Bis-Tris gels (Invitrogen),

1281 transferred to PVDF membrane, blocked for 1 hour in 5\% dry milk in TBST, and incubated with

1282 primary antibodies overnight rocking at $4^{\circ} \mathrm{C}$ (Supplemental Table 2). The next day, the blots 
1283 were washed in TBST three times for 15 minutes, incubated with corresponding HRP-conjugated

1284 secondary antibodies (Abcam ab6721, 1:20,000) for 1 hour at RT, washed in TBST 3 times for

128515 minutes. Images were acquired using film and scanned in to be quantified using image ${ }^{69}$.

1286 Expression was normalized to levels of H2B.

\section{ChIP-seq}

1289 Samples were collected in triplicate, where each replicate came from a plate that started

1290 differentiation at a different time. Samples were harvested by adding $2 \mathrm{~mL}$ of $1 \%$ formaldehyde

1291 to the media for 10 minutes. The reaction was quenched by adding $664.0625 \mathrm{mM}$ of glycine for

12925 minutes. Two washes with ice cold PBS were done before scraping the cells of the plate in

1293 PBS using a cell scraper. The tube was spun down at $2000 \mathrm{rpm}$ for 5 minutes and flash frozen in 1294 liquid nitrogen. The frozen samples were resuspended in $1 \mathrm{~mL}$ lysis buffer $(10 \mathrm{mM}$ Tris, $2 \mathrm{mM}$ 1295 EDTA, and 0.1\% SDS) for 30 minutes on ice and 1-6 wells of a 6-well plate (depending on the 1296 stage and how many cells were in each well) from one batch of cells were combined in the same 1297 tube. The DNA quantity was estimated by using a Qiagen PCR purification kit and the volumes 1298 were normalized to $6.25 \mathrm{ng} / \mu \mathrm{L}$. The cells continued to lyse overnight. The samples were 1299 sonicated on a Bioruptor Pico (23 cycles of 30 seconds on and 30 seconds off) in $0.65 \mathrm{~mL}$ tubes 1300 with $120 \mu \mathrm{L}$ of sample in each tube. The DNA quantity was adjusted to $1 \mu \mathrm{g}$ per sample, diluted 13012 parts dilution buffer to 1 part DNA with a dilution buffer, and incubated with $4 \mu \mathrm{L}$ of H2Bub1 1302 antibody or Igg antibody overnight. It was then incubated with $50 \mu \mathrm{L}$ of protein G Dynabeads 1303 (Invitrogen) for 4 hours at $4^{\circ} \mathrm{C}$ while rocking. The beads were washed twice with low salt buffer, 1304 twice with high salt buffer, twice with $\mathrm{LiCl}$ buffer, and twice with TE buffer (changing tubes 1305 after the first TE wash). Each wash was three minutes long. The immunoprecipitated chromatin 
1306 was eluted from the beads in $85 \mu \mathrm{L}$ of elution buffer two times for 15 minutes at $70^{\circ} \mathrm{C}$. The

1307 immunoprecipitated chromatin was then treated with RNase, underwent reverse cross-linking,

1308 and treated with proteinase $\mathrm{K}$ at $65^{\circ} \mathrm{C}$ overnight. The DNA was purified using a Qiagen PCR

1309 purification kit. qPCR was performed on these samples to verify that the immunoprecipitation

1310 worked using Quantabio PerfeCta syberGreen mix. The samples were run on either a 7900HT

1311 Fast Real-Time PCR Detection System (Applied Biosystems) or a CFX96 Touch Real-Time

1312 (BioRad) in triplicate. The results were analyzed by calculating percent input. The samples that

1313 passed quality control were sequenced at Yale Center for Genome Analysis using NovaSeq.

1314

1315 Buffers

1316 Dilution Buffer: $16.7 \mathrm{mM}$ Tris $\mathrm{pH}=8,167 \mathrm{mN} \mathrm{NaCl}, 1.2 \mathrm{mM}$ EDTA, 0.002\% SDS, $1.1 \%$

1317 Triton, $1 \mathrm{X}$ proteinase inhibitors (Roche), and $5 \mathrm{mM} \mathrm{Na}$ butyrate)

1318

1319 Low Salt Buffer: $20 \mathrm{mM}$ Tris $\mathrm{pH}=8,0.1 \%$ SDS, $1 \%$ Triton, $2 \mathrm{mM}$ EDTA, $150 \mathrm{mM}$ Nacl

1320

1321 High Salt Buffer: $20 \mathrm{mM}$ Tris $\mathrm{pH}=8.0,0.1 \%$ SDS, $1 \%$ Triton, $2 \mathrm{mM}$ EDTA, $500 \mathrm{mM} \mathrm{NaCl}$

1322

1323 LiCl Buffer: $10 \mathrm{mM}$ Tris $\mathrm{pH}=8,250 \mathrm{mM} \mathrm{LiCl}, 1 \mathrm{mM}$ EDTA, 1\% NP40, $1 \%$ Na-deoxycholate 1324

1325 TE Buffer: $10 \mathrm{mM}$ Tris $\mathrm{pH}=8,1 \mathrm{mM}$ EDTA

1326

1327 Elution Buffer: $50 \mathrm{mM}$ Tris $\mathrm{pH}=8,1 \mathrm{mM}$ EDTA, $1 \%$ SDS

1328 


\section{ChIP-seq analysis}

1330 The ChIP-seq reads were trimmed using the FASTX-Toolkit to remove poor quality reads and

1331 were aligned to the human reference genome (hg38) using the BWA Mem algorithm (version

1332 0.7.17). To call peaks, Model-based Analysis of ChIP-seq (MACS) (version 2.1.1.20160309)

1333 was used, where the input was used as the control, and reads were normalized using fold

1334 enrichment ${ }^{70}$. Since H2Bub1 peaks are atypical, the BAMPE option, the no model option, and

1335 the broad option (with a cutoff of 0.2 ) were used. The presence of H2Bub1 near the TSS (-2 Kb,

$1336 \quad 10$ ) as computed using "computeMatrix" in deepTools (version 2.5.0.1) and the genes

1337 were divided into four clusters using kmeans clustering based on the iPSC peaks (Fig. 2a, 2b) or

1338 the wild-type peaks (Fig. 5c, S5c, S8c) ${ }^{71}$. Four clusters were selected unbiasedly by

1339 implementing the elbow method in Python (version 2.7.13). The plots that were generated in

1340 deepTools represent the average of the three replicates. To determine the differentially bound

1341 sites between samples the " $\mathrm{i}$ ind package in $\mathrm{R}$ (version 3. .1) as used ith the ollo ing

1342 parameters: maximum q value of 0.05 and minimum absolute fold change of $1.5^{72,73}$. These

1343 di erentiall ound sites ere matched to the nearest gene using the "ChIPseeker" package in R

1344 (UCSC hg38) $)^{73,74}$. Importantly, a gene was only considered differentially bound (between the

1345 mutants and the wild-type cells) if there was a differentially bound region in the gene in both

1346 mutants. The heat maps were made by selecting the most differentially bound regions in each

1347 gene ontolog ( ) term using the "pheatmap" package in $\mathrm{R}^{73,75}$. The Venn Diagrams were

1348 made using the $\mathrm{R}$ package VennDiagram ${ }^{73,76}$. GO enrichment analysis was done in DAVID

1349 (version 6.8) ${ }^{77,78}$. The gene traces were visualized in the Integrative Genomics Viewer (version

$1350 \quad 2.4 .5)^{79}$

1351 


\section{RNA-seq}

1353 Samples were collected in triplicate, where each replicate came from a plate that started

1354 differentiation at a different time. Each replicate consisted of two wells of a 6-well plate that

1355 were differentiated at the same time. Total RNA was extracted using a Qiagen RNeasy mini kit

1356 with the RNase-Free DNase Set. The mRNA-seq libraries were prepared using a KAPA mRNA

1357 HyperPrep Kit (cat no. 08098123702). Samples were sequenced at Yale Center for Genome

1358 Analysis using NovaSeq.

1359

$1360 \quad$ RNA-seq analysis

1361 The RNA-seq reads were trimmed using the FASTX-Toolkit to remove poor quality reads and

1362 were aligned to the human reference genome (hg38) using Kallisto (version 0.45.0) in R (version

$1363 \quad 3.6 .1)^{73,80}$. Differential expression analysis was done using the Wald test in Sleuth (version

13640.30 .0 ) in $\mathrm{R}$ with the following parameters: maximum $\mathrm{q}$ value of 0.05 and minimum absolute

1365 fold change of $1.5^{73,81}$. BiomaRt (version 3.13) was used to match the transcripts with

1366 genes $^{73,82,83}$. As with ChIP-seq, a gene was only considered to be differentially expressed

1367 (between the mutants and the wild-type cells) if there was a differentially expressed transcript for

1368 the gene in both mutants. The heat maps were made by selecting the most differentially

1369 expressed regions in each term using the "pheatmap" package in $\mathrm{R}^{73,75}$. GO enrichment

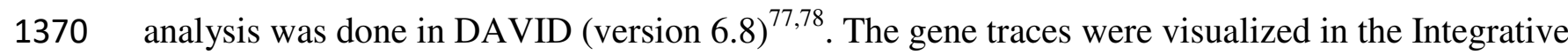

1371 Genomics Viewer (version 2.4.5) 


\section{Analysis of compartment data}

1376 H2Bub1 peaks that overlapped by greater than or equal to 20 positions to a compartment

1377 interval, based on previously collected compartment data, were considered to be part of that

1378 interval ${ }^{38}$. Intervals that had a positive value ere in the " " compartment and intervals that had

1379 a negative value ere in the " " compartment. The three ChIP-seq replicates were compared to

1380 the compartment data separately and then mean and SEM were calculated.

1382 To calculate expected values, 1000 bootstrap replicates were created for each stage of CM

1383 differentiation. A bootstrap replicate was created by sampling the compartment file with

1384 replacement until the bootstrap replicate file had the same number of rows as the H2Bub1 peak

1385 file. By doing this, we generated a "random" collection of intervals from the " " and " "

1386 compartment that was of the same length as the H2Bub1 peak files. The mean and SEM were

1387 calculated on these files to get the expected values.

1389 To calculate $\mathrm{p}$-values between observed and expected, the following equation was used for each

1390 replicate: $p=\frac{\sum_{1}^{n} E_{n}>O}{n}$, where $\mathrm{E}$ is the expected percentage, $\mathrm{O}$ is the observed percentage, and $\mathrm{n}$ 1391 is the bootstrap replicate number. A sample was considered significant if every replicate had a p1392 value less than 0.05 .

1394 To calculate p-values between observed values of different stages, the following equation was 1395 used to calculate a z-score between these two percentages: $z=\frac{p_{1}-p_{2}}{S E}$, where $\mathrm{p}_{1}$ and $\mathrm{p}_{2}$ are the 1396 percents and SE is the standard error. The $\mathrm{z}$ distribution was used to calculate a p-value from the $1397 \quad \mathrm{Z}$-score. All of this analysis was done in R (version 3.6.1) ${ }^{73}$. 


\section{Analysis of histone marks}

1399 H3K27me3 and H3K4me3 peaks were downloaded from the Encode Consortium and generated

1400 by the Bernstein lab ${ }^{39,40}$. Previously collected data on other histone marks from iPSC and CM

1401 stages, as well as the H2Bub1 data from the iPSC and CM stages, were matched to the nearest

1402 gene using the "ChIPseeker" package in R (UCSC hg38) $39,40,73,74$. See the table below for which

1403 ENCODE files were used. For each replicate of every mark at each stage, the presence or

1404 absence of a peak in a gene was recorded in a data frame (1 indicated presence, and 0 indicated

1405 absence). A 2 X2 contingency table was created to record how many genes contained both marks,

1406 how many genes had one mark, and how many genes had neither mark.

1407

1408 To calculate expected values, fixed row and column sums were used. For example, in a $2 \mathrm{X} 2$

1409 contingency table, the expected value of position $(2,2)$ is $E_{(2,2)} \frac{m k}{\text { total }}$, where $\mathrm{m}$ is the column sum

1410 and $\mathrm{k}$ is the row sum ${ }^{84}$. The graph was made by plotting the mean ratios of the observed values to

1411 the expected values. Chi Square test was done to calculate p-values between observed values and

1412 expected values. P-values comparing observed values between stages were calculated using an

1413 unpaired 2-tailed, heteroscedastic t-test. All analyses were done in R (version 3.6.1) ${ }^{73}$.

1414

\begin{tabular}{llll}
\hline Experiment & Bedfile & Target & Tissue \\
\hline ENCSR864LRY & ENCFF289NJD & H3K27me3 & cardiac muscle originated from RUES2 \\
ENCSR000ALU & ENCFF296RYM & H3K27me3 & Human h1-hESC \\
ENCSR652QNW & ENCFF905NTT & H3K4me3 & cardiac muscle originated from RUES2 \\
ENCSR000AMG & ENCFF668YOE & H3K4me3 & Human h1-hESC \\
\hline
\end{tabular}

1415

1416

1417 


\section{Analysis of patterns}

1419 We wanted to investigate the drop off in H2Bub1 on gene bodies between the CMes and CP

1420 stages. Previously, a program was developed that used matrix factorization to investigate patterns

1421 in ChIP-seq and RNA-seq data ${ }^{52}$. Briefly, matrices are created where the rows are genes and the

1422 columns are positions along the gene. The matrices (one for each condition, in this case CMes

1423 and CP) get factored into two matrices, see diagram below. These two matrices when multiplied

1424 together yield the original matrix. The numbers get rearranged in each of these two submatrices

1425 such that genes with similar signals get grouped together.

1426

1427

$\left[\begin{array}{ccc}g_{1}, p_{1} & \cdots & g_{1}, p o_{n} \\ \vdots & \ddots & \vdots \\ g_{n}, p o_{1} & \cdots & g_{n}, p o_{n}\end{array}\right]=\left[\begin{array}{ccc}g_{1}, p a_{1} & \cdots & g_{1}, p a_{n} \\ \vdots & \ddots & \vdots \\ g_{n}, p a_{1} & \cdots & g_{n}, p a_{n}\end{array}\right] *\left[\begin{array}{ccc}p a_{1}, p o_{1} & \cdots & p a_{1}, p o_{n} \\ \vdots & \ddots & \vdots \\ p a_{n}, p o_{1} & \cdots & p a_{n}, p o_{n}\end{array}\right]$, where g is

1428 a gene, po is a position along the gene, and pa is a pattern.

1430 It is unclear if the amount of H2Bub1 at a particular location is important, or whether the

1431 presence of $\mathrm{H} 2 \mathrm{Bub} 1$ at a particular location is important. Therefore, we created two versions of

1432 these matrices.

1433

1434 Version one considers both distribution and amount. To create this version, we used

1435 "computeMatrix" in deepTools (version 2.5.0.1) ${ }^{71}$. We used the scale regions option to be able to

1436 analyze the entire gene. We limited our search from $2 \mathrm{~Kb}$ upstream of the TSS to $2 \mathrm{~Kb}$

1437 downstream of the TES. We used 5000 for the region body length, which gave us 900 bins. We

1438 combined the three replicates together by averaging in $\mathrm{R}$ (version 3.6.1) ${ }^{73}$.

1439 
1440 Version two considers only distribution. To create this version, we went line by line in the

1441 H2Bub1 peak files using R (version 3.6.1) code $^{73}$. For each peak, we used interval searching to

1442 search through the ENSEMBL genes (this file was sorted by chromosome and the genes were in

1443 order based on position on the chromosome) to find all of the ENSEMBL genes each peak

1444 overlapped with. We divided each ENSEMBL gene into 100 bins of equal size. A peak was

1445 considered to be in a bin if it overlapped with more than a quarter of the bin. This created a 3d

1446 matrix, where the rows were genes, the columns were positions along the gene, and the third

1447 dimension was the replicates. To combine the replicates, a peak was considered present if it was

1448 present in a given bin of a given gene in at least two replicates.

1449

1450 We ran these four matrices (CMes version 1, CMes version 2, CP version 1, and CP version 2)

1451 through the previously published pipeline. After some tweaking of the $\mathrm{K}$ values to get a $\mathrm{K}$ value

1452 that is more representative of the global minimum, we decided to make $\mathrm{K}$ go from 2-8 for

1453 version 1 and $\mathrm{K}$ go from 2-7 for version 2 . K represents the number of patterns. The $\mathrm{K}$ values

1454 were manually checked. In both cases, no common patterns emerged. The K value for CMes was

1455 seven and for $\mathrm{CP}$ was five in version 1 . The $\mathrm{K}$ value for CMes was six and for $\mathrm{CP}$ was four in

1456 version 2. This program produced the graphs in Fig. S4a. To create Fig. S4b, the genes in each

1457 pattern from CMes in each version were compared to the differentially occupied genes identified

1458 using diffbind.

1459

1460 To create Fig. S4c, the genes in each pattern were combined with the RNA-seq data. The

1461 transcript with the highest expression level in the CMes stage for a given gene was used for both

1462 the CMes stage and the CP stage. Expression levels for each gene in each pattern at both stages 
1463 were recorded in a matrix in $\mathrm{R}$ (version 3.6.1) ${ }^{73}$. The box and whisker plots were plotted using $1464 \operatorname{ggplot} 2^{73,85}$

\section{Mouse calcium qRT-PCR}

1467 e11.5 mouse hearts were dissected and flash frozen in liquid nitrogen separately. After

1468 genotyping, 3-10 hearts were pooled together to form each sample. RNA was extracted with the

1469 Qiagen RNeasy Plus mini kit. cDNA was synthesized with Quantabio qScript cDNA SuperMix.

1470 qPCR was done using Quantabio PerfeCta syberGreen mix on a CFX96 Touch Real-Time

1471 (BioRad) in biological duplicates and technical triplicates. See Supplemental Table 1 for primers.

1472 Expression was normalized to the ribosomal protein $18 s$.

1473

\section{Movies}

1475 Movies of the CM stage cells were produced using bright-field imaging on the Zeiss Observer 1476 Z1. We collected 50 frames, 1 per second.

\section{Metagene analysis}

1479 We first assembled a list of regions. We decided to focus on calcium signaling genes and 1480 sarcomeric genes, since those are the genes that are differentially expressed between the ChIP1481 seq and RNA-seq data. We combined the sets of genes that were selectively maintained and 1482 differentially expressed into two bed files (one for calcium signaling genes and one for

1483 sarcomeric genes). We used the R package metagene2 (version 1.8.0) to create the plots with the 1484 following parameters: normalize with RPM, alpha $=1$, and paired_end is true ${ }^{73,86}$. 
1486 To generate "random" set of genes, we fit the lengths of the genes in the calcium signaling bed

1487 file and the sarcomeric gene bed file to the poisson function in $\mathrm{R}$ (version 3.6.1) ${ }^{73}$. We then

1488 generated random numbers (the same number as there are lines in each bed file) from that

1489 distribution. For each random number, we found all of the down regulated genes in $U B E 2 B^{-/ 1} 1$

1490 that are within $10 \%$ of that number. We then kept picking a gene at random until one was

1491 downregulated in both mutants. We repeated the procedure for upregulated and non-regulated

1492 genes. We created 10 sets of each (upregulated calcium signaling, downregulated calcium

1493 signaling, non-regulated calcium signaling, upregulated sarcomeric genes, downregulated

1494 sarcomeric genes, and non-regulated sarcomeric genes). To verify that there was no

1495 accumulation near the center, these 60 graphs were manually inspected.

1496

1497 Transcriptional efficiency

1498 To calculate transcriptional efficiency, we used the R package CoverageView (version

$14991.30 .0)^{73,87}$. We modified the source code of draw.heatmap to produce a pdf file to make it

1500 compatible with the machine it was being run on. We used 100 windows, normalized with RPM,

1501 and presented the graph as a $\log 2$ ratio. We produced one graph per replicate per mutant. Since all

1502 of the replicates look similar and both mutants look similar, we show one representative image.

1503 We used the same set of "random" genes generated for the metagene analysis. We manually

1504 inspected the 360 resulting graphs (ten replicates of "random" genes for three classes of genes

1505 (upregulated, downregulated, and non-regulated), for two bed files (calcium signaling genes and

1506 sarcomeric genes) for three replicates of two mutant cell lines). The gene traces were visualized

1507 in the Integrative Genomics Viewer (version 2.4.5) ${ }^{79}$.

1508 


\section{mESC and MEF analysis}

1510 We downloaded the raw data from previously published work and put it through our ChIP-seq

1511 and RNA-seq pipelines described in the ChIP-seq and RNA-seq analysis sections ${ }^{32}$.To determine

1512 the tissue-specific genes for the MEFs, we used the Wald test in Sleuth (version 0.30.0)

1513 (maximum q value of 0.05 and minimum absolute fold change of 1.5) to compare the wild-type

1514 MEFs to the wild-type mESCs ${ }^{73,81}$. BiomaRt was used to match the transcripts with genes ${ }^{73,82,83}$.

1515 GO enrichment analysis was done using DAVID (version 6.8) on the genes upregulated in the

1516 MEFs compared to the mESCs ${ }^{77,78}$. The GO terms were related to ions, adhesion, and/or ECM.

1517 Thus, we created metagenes for genes in those three categories following the pipeline described

1518 in the metagene section. Since this group did not knockout a component of the RNF20-complex,

1519 we did not think it was necessary to create "random" genes representing the upregulated,

1520 downregulated, or non-regulated genes. Therefore, we created 20 "random" gene sets for ions,

152120 "random" gene sets for adhesion, and 20 "random" gene sets for ECM.

1522

\section{Statistics and reproducibility}

1524 Graphs and statistics were either done in R (version 3.6.1) or Prism (version 9.1.0) ${ }^{73}$. All

1525 experiments were done in replicate, with the number clearly indicated on the figure or in the

1526 figure legend. All statistical tests are defined in the figure legend and any non-traditional tests

1527 are described in detail in the appropriate section of the online methods. Multiple comparison

1528 adjustments were done using Bonferroni method. Biological replicates are samples analyzed

1529 from distinct samples. To account for off-target effects due to CRISPR, we created two cell lines

1530 for each mutant. For mouse experiments, since multiple mice were pooled together into a 
1531 replicate, both sexes were mixed together in each sample. Additionally, littermate controls were

1532 used. The iPSC experiments are all done in a male cell line.

1533

1534 Data Availability

1535 Requests for mice, induced pluripotent stem cell lines, or reagents will be granted by the 1536 corresponding author. The ChIP-seq and RNA-seq data will be deposited in GEO and we will 1537 list the accession numbers here. The other ChIP-seq and RNA-seq data sets that we used are 1538 available from the following resources. The A and B compartment data are available under GEO 1539 accession GSE106690. The histone mark data are available on ENCODE; see online methods for 1540 ENCODE accessions. The MEF data are available under GEO accession GSE153587.

\section{Code Availability}

1543 All software used in this study is publicly available. The code for figures and analysis is 1544 available upon request. 


\section{References}

1546

1547

1548

1549

1550

1551

1552

1553

1554

1555

1556

1557

1558

1559

1560

1561

1562

1563

1564

1565

1566

1567

1568

1569

1570

1571

1572

1573

1574

1575

1576

1577

1578

1579

1580

1581

1582

1583

1584

1585

1586

1587

32. Wang, F. et al. Histone H2Bub1 deubiquitylation is essential for mouse development, but does not regulate global RNA polymerase II transcription. Cell Death Differ (2021).

37. Lian, X. et al. Directed cardiomyocyte differentiation from human pluripotent stem cells by modulating Wnt/beta-catenin signaling under fully defined conditions. Nat Protoc $\mathbf{8}$, 162-75 (2013).

38. Bertero, A. et al. Dynamics of genome reorganization during human cardiogenesis reveal an RBM20-dependent splicing factory. Nat Commun 10, 1538 (2019).

39. Inoue, F. et al. A systematic comparison reveals substantial differences in chromosomal versus episomal encoding of enhancer activity. Genome Res 27, 38-52 (2017).

40. Kazachenka, A. et al. Identification, Characterization, and Heritability of Murine Metastable Epialleles: Implications for Non-genetic Inheritance. Cell 175, 1259-1271 e13 (2018).

52. Zhang, L. \& Zhang, S. Learning common and specific patterns from data of multiple interrelated biological scenarios with matrix factorization. Nucleic Acids Res 47, 66066617 (2019).

68. Chen, Y. et al. Engineering Human Stem Cell Lines with Inducible Gene Knockout using CRISPR/Cas9. Cell Stem Cell 17, 233-44 (2015).

69. Schneider, C.A., Rasband, W.S. \& Eliceiri, K.W. NIH Image to ImageJ: 25 years of image analysis. Nat Methods 9, 671-5 (2012).

70. Zhang, Y. et al. Model-based analysis of ChIP-Seq (MACS). Genome Biol 9, R137 (2008).

71. Ramirez, F. et al. deepTools2: a next generation web server for deep-sequencing data analysis. Nucleic Acids Res 44, W160-5 (2016).

72. Stark, R. \& Brown, G. DiffBind: differential binding analysis of ChIP-Seq peak data. Bioconductor http://bioconductor.org/packages/release/bioc/vignettes/DiffBind/inst/doc/DiffBind.pd f. (2011).

73. R Core Team. R: A language and environment for statistical computing. (R Foundation for Statistical Computing, Vienna, Austria, 2019).

74. Yu, G., Wang, L.G. \& He, Q.Y. ChIPseeker: an R/Bioconductor package for ChIP peak annotation, comparison and visualization. Bioinformatics 31, 2382-3 (2015).

75. Kolde, R. pheatmap: Pretty Heatmaps. R package version 1.0.8. https://CRAN.Rproject.org/package=pheatmap. (2015).

76. Chen, H. \& Boutros, P.C. VennDiagram: a package for the generation of highlycustomizable Venn and Euler diagrams in R. BMC Bioinformatics 12, 35 (2011).

77. Huang da, W., Sherman, B.T. \& Lempicki, R.A. Systematic and integrative analysis of large gene lists using DAVID bioinformatics resources. Nat Protoc 4, 44-57 (2009).

78. Huang da, W., Sherman, B.T. \& Lempicki, R.A. Bioinformatics enrichment tools: paths toward the comprehensive functional analysis of large gene lists. Nucleic Acids Res 37, 113 (2009).

79. Thorvaldsdottir, H., Robinson, J.T. \& Mesirov, J.P. Integrative Genomics Viewer (IGV): high-performance genomics data visualization and exploration. Brief Bioinform 14, 17892 (2013). 
1588 80. Bray, N.L., Pimentel, H., Melsted, P. \& Pachter, L. Near-optimal probabilistic RNA-seq quantification. Nat Biotechnol 34, 525-7 (2016).

81. Pimentel, H., Bray, N.L., Puente, S., Melsted, P. \& Pachter, L. Differential analysis of RNAseq incorporating quantification uncertainty. Nat Methods 14, 687-690 (2017).

82. Durinck, S. et al. BioMart and Bioconductor: a powerful link between biological databases and microarray data analysis. Bioinformatics 21, 3439-40 (2005).

83. Durinck, S., Spellman, P.T., Birney, E. \& Huber, W. Mapping identifiers for the integration of genomic datasets with the R/Bioconductor package biomaRt. Nat Protoc 4, 1184-91 (2009).

84. Fu, A.Q. \& Adryan, B. Scoring overlapping and adjacent signals from genome-wide ChIP and DamID assays. Mol Biosyst 5, 1429-38 (2009).

85. Wickham, H. ggplot2: Elegant Graphics for Data Analysis. (Springer-Verlag New York. , 2016).

86. Fournier, E., Joly Beauparlant, C., Lippens, C. \& Droit, A. metagene2: A package to produce metagene plots. $R$ package version 1.8.0. (2021).

1604

87. Lowry, E. CoverageView: Coverage visualization package for R. R package version 1.30.0. (2021). 


\section{Supplementary Files}

This is a list of supplementary files associated with this preprint. Click to download.

- SupplementalData6UBE2BRNAseq.xlsx

- SupplementalData4RNF20RNAseq.xlsx

- Movie4RNF202NotBeating.mov

- Movie3RNF201NotBeating.mov

- SupplementalData2WildTypeRNAseq.xIsx

- SupplementalData5UBE2BChIPseq.xIsx

- SupplementalData3RNF20ChIPseq.xlsx

- Movie2RNF201Beating.mov

- Movie6UBE2B1NotBeating.mov

- Movie8UBE2B2NotBeating.mov

- SupplementalData1WildTypeChIPseq.xlsx

- Movie1wildtype.mov

- Movie5UBE2B1Beating.mov

- Movie7UBE2B2Beating.mov 